\title{
An update on the genera Ascobolus and Saccobolus with keys and descriptions of three coprophilous species, new to Italy
}

\author{
Doveri $\mathbf{F}^{1}$ \\ ${ }^{1}$ via dei Funaioli 22, I 57126 Livorno, Italy, f.doveri@sysnet.it
}

Doveri F 2014 - An update on the genera Ascobolus and Saccobolus with keys and descriptions of three coprophilous species, new to Italy. Mycosphere 5(1), 86-135, Doi 10.5943/mycosphere/5/1/4

\begin{abstract}
Ascobolus aglaosporus, Ascobolus sacchariferus and Saccobolus obscurus are described and discussed. All author's collections of 19 Ascobolus and 13 Saccobolus so far known from dung in Italy are listed, and each species illustrated by colour photos. Updated keys to coprophilous Ascobolus and Saccobolus species from Italy are provided.
\end{abstract}

Key words - dichotomous keys - dung - records

\section{Introduction}

The present update follows Doveri et al. (2000) and Doveri (2007, 2011), and follows Doveri's (2004) monograph on coprophilous ascomycetes and basidiomycetes from Italy. Doveri (2004) described and keyed out 15 Ascobolus and 12 Saccobolus species isolated from a variety of herbivore dung, exceptionally from carnivore, and briefly redescribed and inserted in keys all species published by others after van Brummelen's (1967) world-monograph of the genera Ascobolus Pers. and Saccobolus Boud. Doveri (2007, 2012) updated his previous key to coprophilous Pezizales J. Schröt. and Thelebolales P.F. Cannon and described Ascobolus reticulatus Brumm. and A. perforatus Brumm. as species new to Italy from dung. Doveri (2011) listed all coprophilous species of Ascobolus and Saccobolus reported from Italy in his previous papers and discussed their frequency of occurrence on different dung sources.

Since Doveri (2004), six new Ascobolus species have been introduced (A. bonaerensis Dokm. et al., A. calesco A.E. Bell \& Mahoney, A. campanensis Dokm. et al., A. dadei A.E. Bell \& Mahoney, A. nairobiensis Mungai \& K.D. Hyde, A. tsavoensis Mungai \& K.D. Hyde), all from dung. We refer to the respective protologues (Dokmetzian et al. 2004, Bell 2005, Bell \& Mahoney 2007, Mungai et al. 2012) for their systematic placement in sections according to van Brummelen (1967). No new species of Saccobolus has been published after Doveri (2004).

Coprophily is a fairly common style of life in mycobiota, shared by most Ascobolus and Saccobolus. At the present I have cultured in damp chambers 484 dung samples of 45 animal species, providing 203 collections (10\%) of Ascobolus spp. and 184 collections (9\%) of Saccobolus spp. out of 2092 collections of 214 Ascomycota spp.

The aim of this work is to fully describe three species new to Italy and to update records of all coprophilous Ascobolus and Saccobolus spp. so far known in this country. 


\section{Materials \& Methods}

The three species new to Italy were obtained from different types of dung cultured in nonaxenic damp chambers, and studied according to Richardson \& Watling (1997) and Richardson (2001a), slightly modified by Doveri (2004).

Abbreviations: $\mathrm{AMB}=$ Herbarium of Bresadola Mycological Association; CLSM $=$ author's personal herbarium; d.c. $=$ damp chamber culture; $\mathrm{MCVE}=$ Herbarium of Venetian Civic Museum.

\section{Taxonomy}

Ascobolus aglaosporus Heimerl, K.K. Ober-Realsch. Bez. Sechs. Wien 15: 141889.

Figs $1-10$

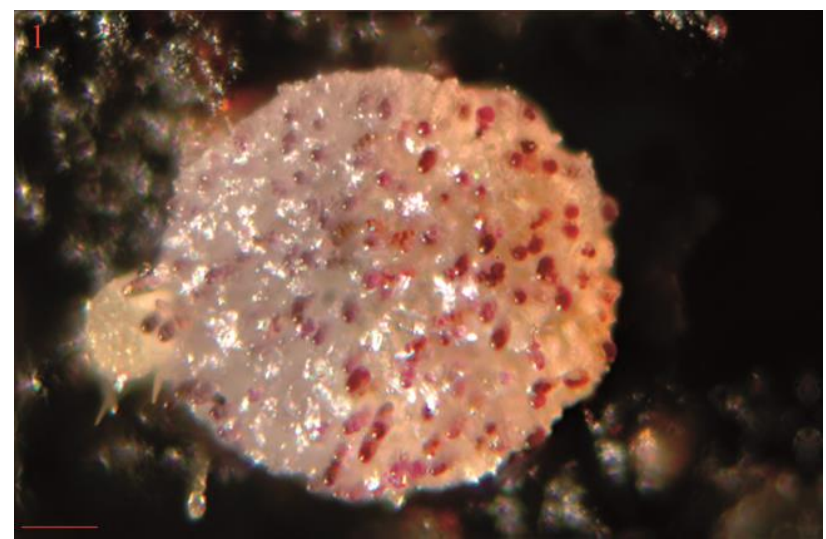

Fig. 1 - Ascobolus aglaosporus. Ascoma on dung. - Bar $=70 \mu \mathrm{m}$.

Material examined - Italy, Tuscany, Livorno, Quercianella, $50 \mathrm{~m}$ a.s.1., three superficial, gregarious specimens on fallow deer (Dama dama) dung in d.c., 22 Nov 2012, F. Doveri, CLSM 008.12 .

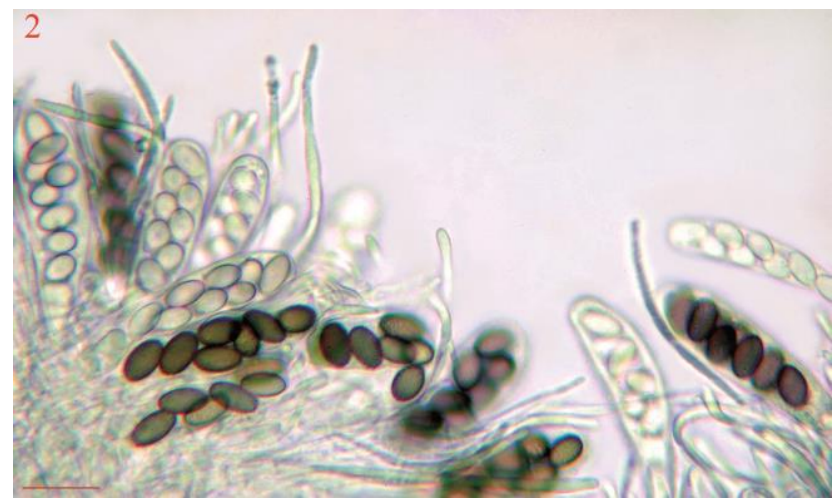

Fig. 2 - Ascobolus aglaosporus. Detail of hymenial surface. - Bar $=20 \mu \mathrm{m}$.

Ascomata $0.35-0.45 \mathrm{~mm}$ diam., sessile, pulvinate, with a scarcely differentiated margin. Outer surface whitish, smooth. Disc slightly convex, the same colour, violet-dotted at maturity due to the protruding asci. Subhymenium a textura globulosa-angularis of polygonal to roundish cells, 4-5 $\mu \mathrm{m}$ diam., interspaced with some elongated cells. Medullary excipulum and ectal excipulum scarcely differentiated from each other, a textura globulosa-angularis of hyaline to pale yellowish, roundish or polygonal cells, 5-10 $\times 6-12 \mu \mathrm{m}$. Paraphyses exceeding the asci, not embedded in a gelatinous material, simple or often branched at their base, filiform, 2-4 $\mu \mathrm{m}$ diam., septate, containing some hyaline vacuoles, curved, usually slightly enlarged at the apex, sometimes clearly inflated. Asci 8-spored, sometimes 3-6-spored, operculate, weakly amyloid, 100-115 × 17-20 $\mu \mathrm{m}$, 


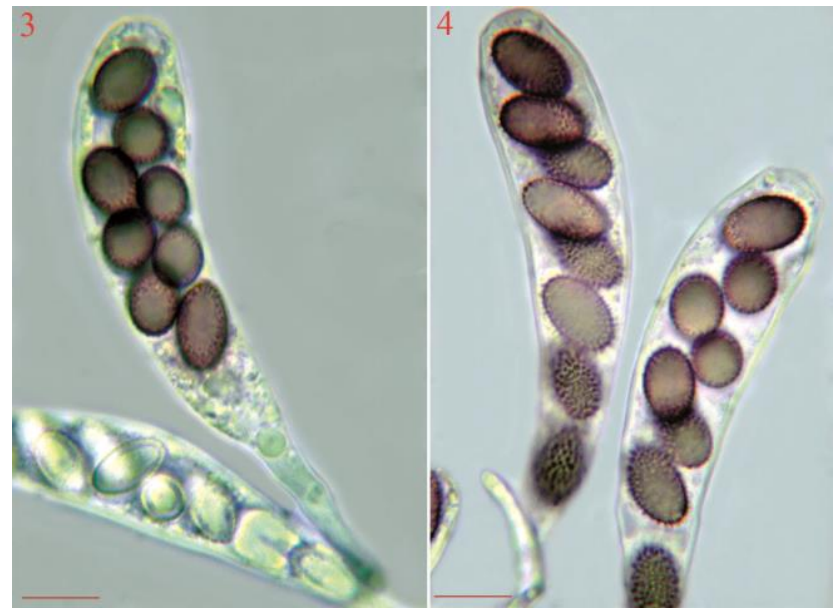

Figs 3-4 - Ascobolus aglaosporus. Asci with ascospores in different stages of maturity. - Bars $=10$ $\mu \mathrm{m}$.

cylindric-claviform, somewhat flattened at the apices, short-stalked. Ascospores irregularly biseriate at maturity, surrounded by a narrow, ephemeral gelatinous sheath, (13-) 13.5-15 (-16) $\times(7.5-) 8(-$ 9) $\mu \mathrm{m}$, ellipsoidal or narrowly ellipsoidal $(\mathrm{Q}=1.62-2.00$; average $\mathrm{Q}=1.74)$, symmetrical, roundish at the ends, thin-walled, hyaline at first, violet later, finally pale brown, ornamented with dense, usually isolated warts and spines, up to $0.5 \mu \mathrm{m}$ wide, $0.5-1 \mu \mathrm{m}$ high.

Notes - Van Brummelen (1967) erected section Heimerlia to accomodate two species, A. aglaosporus and A. candidus J. Schröt., characterised by small, white, pulvinate or lenticular gymnohymenial ascomata (hymenium exposed from the very beginning), with an even margin lacking a growing zone. In this section the excipulum is scarcely developed, paraphyses are not pigmented, asci are elongated and amyloid, ascospores are symmetrical, ellipsoidal, usually echinulate or warted.

A. candidus differs from A. aglaosporus in having smooth and smaller $(11-13 \times 6-8 \mu \mathrm{m})$ ascospores (Schroeter 1893, van Brummelen 1967). According to van Brummelen (1967) A. candidus ss. Seaver (1928) is A. aglaosporus.

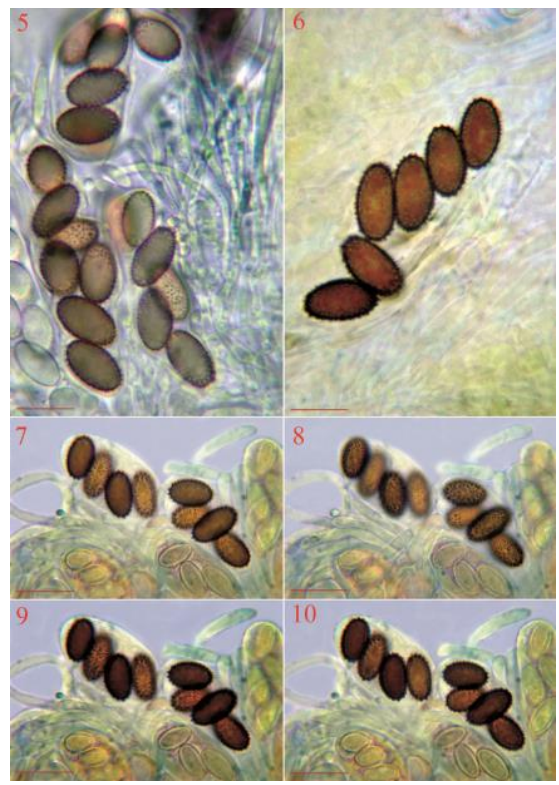

Figs 5-10 - Ascobolus aglaosporus. 5-6 Ascospores in dfferent stages of maturity ( $6=$ in Melzer). 7-10 (Melzer) Ascospores inside asci in four different focuses. - Bars 5-6 $=10 \mu \mathrm{m} .7-10=5 \mu \mathrm{m}$. 
Ascospores of our collection of A. aglaosporus from fallow deer dung are somewhat smaller than those described in the protologue (Heimerl 1889) from deer dung, and also smaller than those described by others (van Brummelen 1967, Raitviir \& Prokhorov 1988, Prokhorov \& Raitviir 1991, Valldosera \& Guarro 1992) from domestic (horse, goat, rabbit, sheep) or wild (bison, ibex, deer) herbivore dung. Most collections of A. aglaosporus in North America (Parker 1979) are from horse $(50 \%)$ and deer $(25 \%)$ dung, minor percentages from cow, rabbit and sheep.

Ascobolus sacchariferus Brumm., Persoonia suppl. vol. 1: 1221967.

Figs 11-19

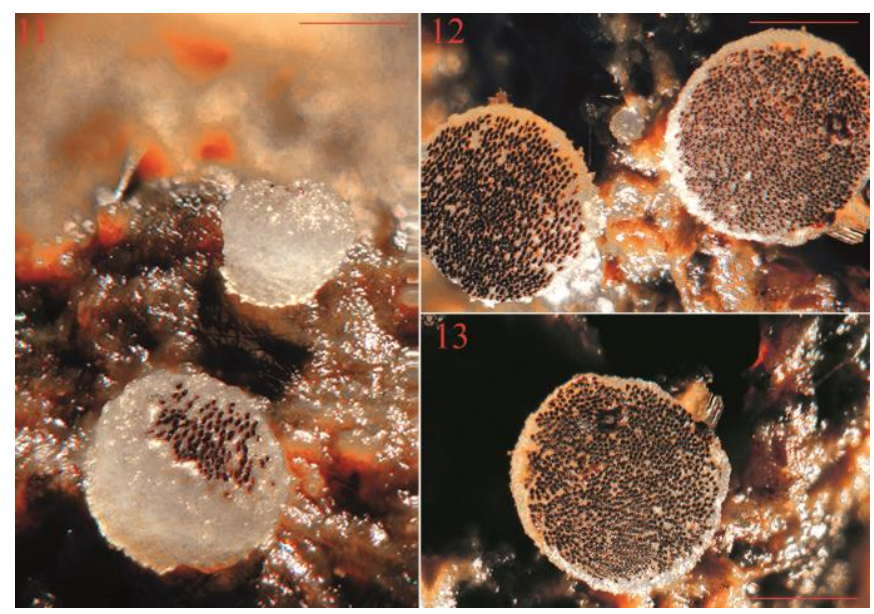

Figs 11-13 - Ascobolus sacchariferus. Ascomata on dung. - Bars $=500 \mu \mathrm{m}$.

Material examined - Italy, Veneto, Venice, Caroman, $0 \mathrm{~m}$ a.s.l., about fifty supericial, gregarious specimens on peewit gull (Larus sp.) dung in d.c., 18 Apr 2010, L. Levorato, CLSM 004.11 .

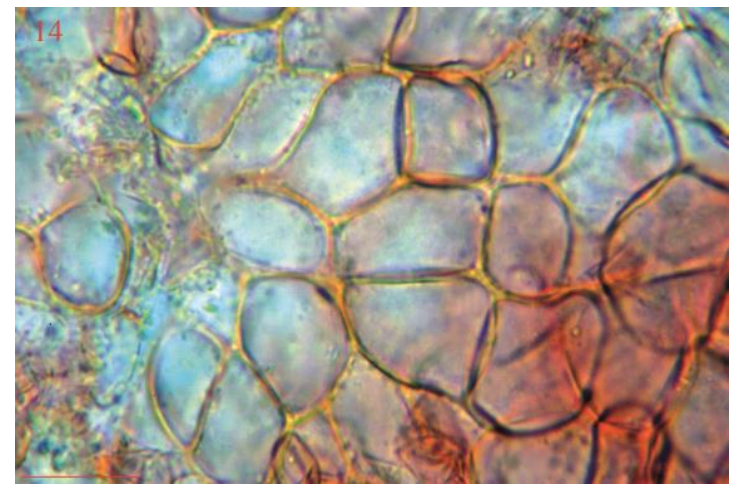

Fig 14 - Ascobolus sacchariferus. Detail of ectal excipulum at the base. - Bar $=25 \mu \mathrm{m}$.

Ascomata $0.75-1.25 \mathrm{~mm}$ diam., sessile, subglobose to hemispherical in the early stages, obconical later, finally almost flattened, with a distinct, narrow margin. Outer surface dirty white, finely granulose. Hymenial surface plane or slightly concave, the same colour, violet-dotted at maturity due to the protruding asci. Subhymenium a textura angularis of polygonal to cylindrical cells, 2.5-5 $\mu \mathrm{m}$ diam. Medullary excipulum a textura globulosa-angularis, formed of roundish or polygonal cells, 6-9 $\mu \mathrm{m}$ diam., mixed with broadly ellipsoidal hyphae, constricted at the septa. Ectal excipulum a textura prismatica in the upper portion of the receptacle, formed of more or less cylindrical cells, parallel to the margin, a textura globulosa-angularis of hyaline, roundish or polygonal cells, $12-30 \times 10-27 \mu \mathrm{m}$, in the lower portion. Marginal cells vertical, cylindrical, with rounded apices. Numerous septate, wavy, hyphoid hairs, up to $4 \mu \mathrm{m}$ diam., arise from the base of 


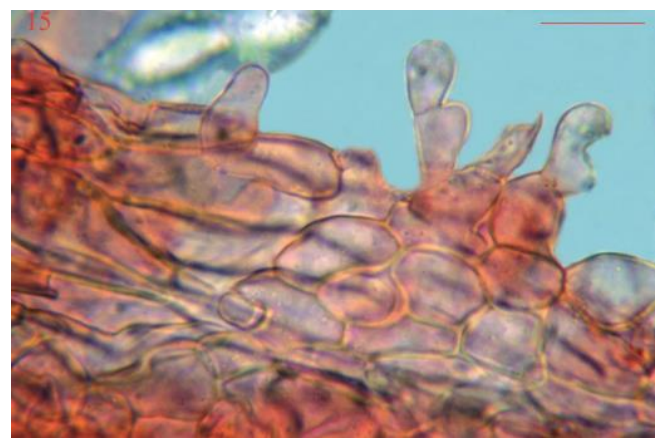

Fig 15 - Ascobolus sacchariferus. Detail of ectal excipulum at the margin. - Bar $=25 \mu \mathrm{m}$.

apothecia. Paraphyses exceeding the asci, embedded in a thin, hyaline gelatinous material, simple or often branched at some level, filiform, 2-2.5 $\mu \mathrm{m}$ diam., septate, containing some hyaline vacuoles, curved, sometimes inflated up to $8 \mu \mathrm{m}$ diam. or diverticulate at the tips, enlarged at their bases up to $7 \mu \mathrm{m}$. Asci 8-spored, operculate, weakly amyloid, 190-225 × 19-22 $\mu \mathrm{m}$, cylindric-claviform, somewhat flattened at the apices, fairly long-stalked. Ascospores biseriate at maturity, covered with a unilateral gelatinous sheath, 18-19 $(-20) \times 8.5-9.5 \mu \mathrm{m}$, narrowly ellipsoidal $(\mathrm{Q}=1.94-2.37 ; \mathrm{Q}=$ 2.10), symmetrical, roundish at the ends, thick-walled, hyaline at first, becoming violet, finally purple, with an episporium ornamented with numerous, thin, longitudinal cracks, which sometimes anastomose.

Notes - In sect. Ascobolus ss. van Brummelen (1967) (cleistohymenial, quite early opening, often finally flattened ascomata, with cylindric-clavate asci and usually striate or warted ascospores), A. sacchariferus is defined by white, furfuraceous receptacles with a well developed margin, and ellipsoidal ascospores with longitudinal, anastomosing crevices (van Brummelen 1967). It is a fairly common species, particularly in north temperate areas (Richardson 2007), and I have found 46 records worldwide, particularly (80\%) from wild herbivore dung (Eckblad 1968, Minoura et al. 1978, Ghadge \& Patil 1988, Prokhorov 1989, Kutorga 2000, Richardson 2008), mostly (52\%) from cervine pellets (Paulsen \& Dissing 1979, Jahn 1997, Richardson 1998), but also from several domestic herbivore dung (Moravec 1970, Aas 1978a, Prokhorov 1989), and mine from bird droppings.

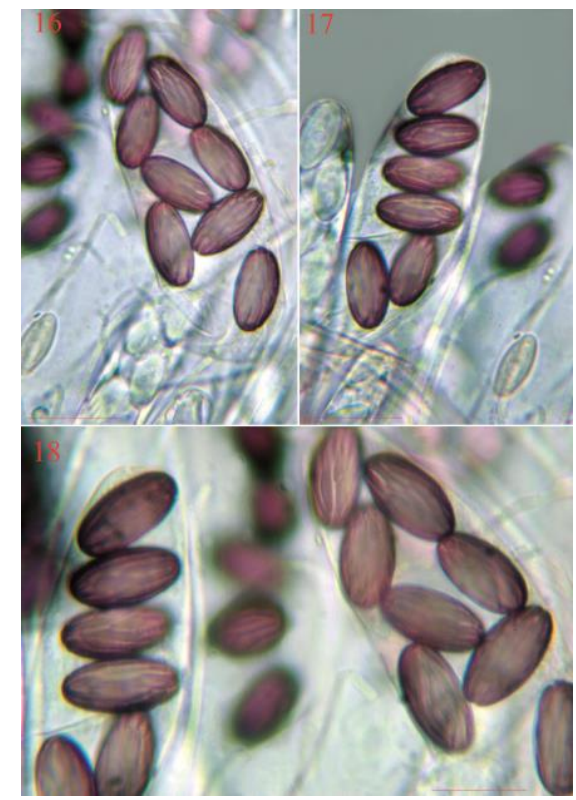

Figs 16-18 - Ascobolus sacchariferus. Asci with ascospores. - Bars 16-17 $=20 \mu \mathrm{m} .18=15 \mu \mathrm{m}$. 


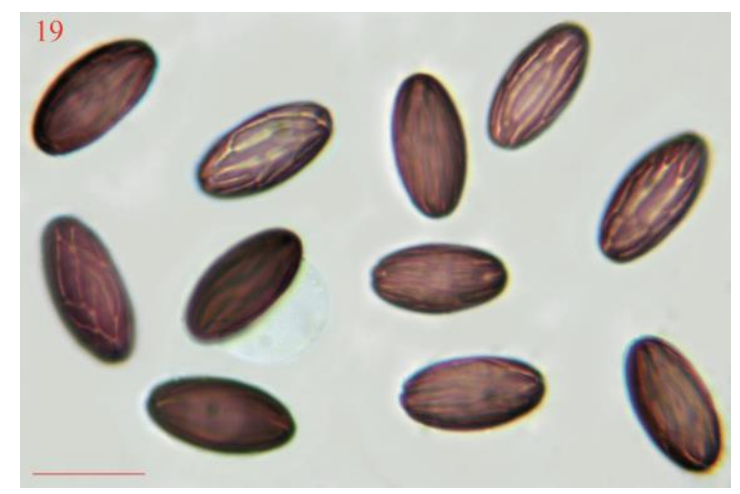

Fig $19-$ Ascobolus sacchariferus. Ascospores. - Bar $=12 \mu \mathrm{m}$.

Based on a collection from deer dung, Moravec (1970) erected A. sacchariferus f. roseogriseus, which differs from the type form only in having grey-ochraceous ascomata with a pale pink disc. The same form, from the same substrate, was also recorded by Paulsen \& Dissing (1979).

A. sacchariferus f. sacchariferus is similar to two other white species in the same section, $A$. brantophilus Dissing and A. albidus, but both have smooth receptacles with an undefined margin and somewhat larger ascospores, besides A. brantophilus has ascospores with very few longitudinal crevices and always has been recorded from high latitude areas and goose dung (Dissing 1989, Richardson 2004, 2007). A. albidus also differs from A. sacchariferus in having ascospores with occasionally anastomosing crevices, and a wider choice of substrate: it is a very common, widespread species, particularly in north temperate areas (van Brummelen 1967, Richardson 2007), where colonises most wild and domestic herbivore dungs, rarely carnivore dung. We have found 169 records worldwide, mine from Italy included, 24\% from horse (Svrček 1959, Julich 1968, Bednarczyk 1974, Raitviir \& Prokhorov 1988, Jahn 1997, Richardson 2011), 24\% from cervine (20\% roe deer) (Svrček 1957, Paulsen \& Dissing 1979, Caillet \& Moyne 1982, Prokhorov 1989, Moyne \& Petit 2007, Richardson 2008), 17\% from sheep (Lohmeyer 1995; Richardson 2004, 2005, 2011; De Meulder 2007), 12\% from leporid (hare and rabbit) (Marchal 1895, Barrasa Gonzales 1985, Richardson 2005), 8\% from cattle dung (Crouan \& Crouan 1858, Velenovský 1934, Svrček 1962, Eckblad 1968, Ranalli \& Cinto 1972, De Meulder 2000), and minor percentages from other types of dung. These results fully match Richardson (2007).

Saccobolus obscurus (Cooke) W. Phillips, Brit. Discom.: 2951887.

Figs 20-25 三Ascobolus obscurus Cooke, Grevillea 4: 1121876.

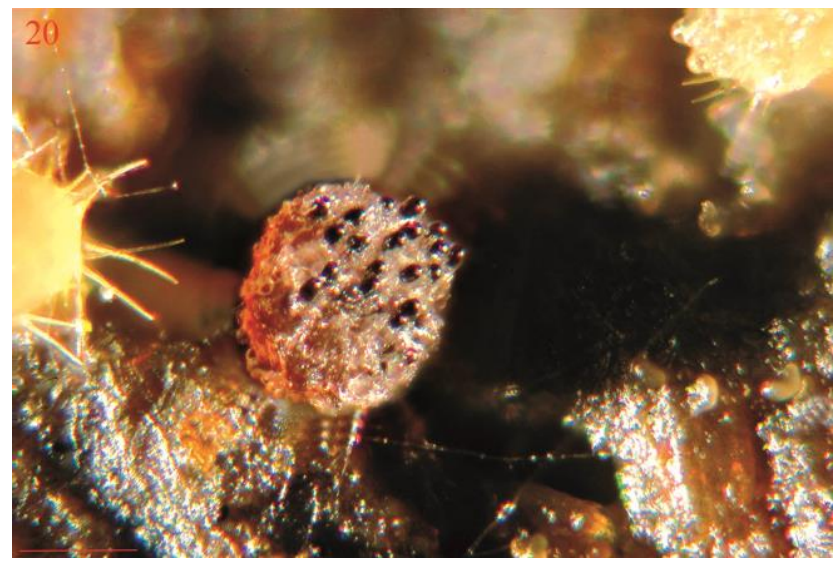

Fig 20 - Saccobolus obscurus. Ascoma on dung between two specimens of Lasiobolus cuniculi. Bar $=150 \mu \mathrm{m}$. 


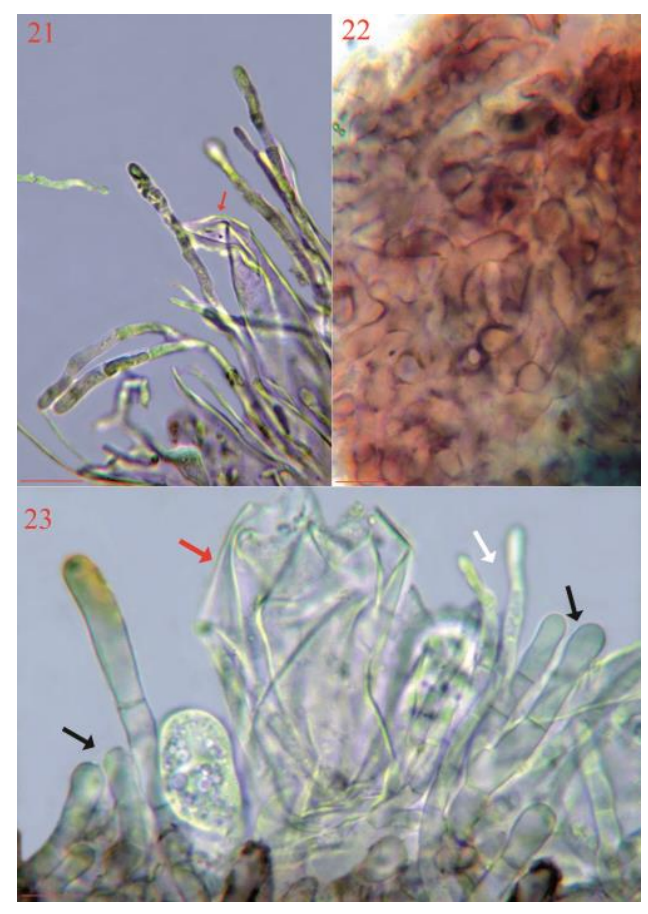

Figs 21-23 - Saccobolus obscurus. 21 Paraphyses exceeding an empty ascus (arrow). 22 Detail of ectal excipulum. 23 Elongated cells of the excipular margin (black arrows), an empty ascus (red arrow), and paraphyses (white arrow). - Bars 21, $23=10 \mu \mathrm{m} .22=15 \mu \mathrm{m}$.

Material examined - Italy, Tuscany, Elba island, $0 \mathrm{~m}$ a.s.l., about ten, supericial, gregarious specimens on sheep (Ovis aries) dung in d.c., 9 Apr 2011, L. Levorato, CLSM 005.12; Elba island, $0 \mathrm{~m}$ a.s.1., on wild rabbit (Oryctolagus cuniculus) dung in d.c., 9 Apr 2011, L. Levorato, CLSM 005.12 bis; Livorno, Quercianella, $50 \mathrm{~m}$ a.s.l., on fallow deer (Dama dama) dung, 22 Nov 2012, F. Doveri, CLSM 005.12 ter.

Apothecia 200-300 $\mu \mathrm{m}$ diam., sessile, pulvinate, finally discoidal, membranous, smooth, pale brown. Disc slightly paler, dark dotted due to the protruding asci. Margin undifferentiated. Subhymenium and medullary excipulum scarcely differentiated from the ectal excipulum, with paler hyphae. Ectal excipulum a textura intricata (globulosa at small intervals), formed of elongated cells, 3-5 $\mu \mathrm{m}$ diam., swelling sometimes up to $9 \mu \mathrm{m}$ diam. and smeared with an intercellular purple-brown to brown pigment. Some elongated cells, with globose or claviform ends, similar to the paraphyses, but with thicker walls, are observable at the margin. Paraphyses cylindricfiliform, septate, often branched at the base, sometimes diverticulate, exceeding the asci, often curved at the apex, 1.5-2.5 $\mu \mathrm{m}$ diam., inflated up to $3.5 \mu \mathrm{m}$ at the tips. Asci ephemeral, 100-110 $\times$ 18-24 $\mu \mathrm{m}, 8$-spored, diffusely amyloid, operculate, clavate, thick-walled, rounded to slightly flattened at the apex, with a fairly long, lobate stalk. Ascospores (12-) 12.5-13 $(-13.5) \times 7-8 \mu \mathrm{m}$ (ornamentations excluded), ellipsoidal or ovoidal to broadly fusiform $(\mathrm{Q}=1.50-1.85 ; \mathrm{Q}=1.67$ ), sometimes slightly inequilateral, usually with subacute, somewhat rounded ends, hyaline and thickwalled in the early stages, becoming violet, finally purple-brown, ornamented with short, sometimes merging warts, $0.5-2 \mu \mathrm{m}$ diam., crowded inside the ascus, firmly cemented and arranged in clusters (24-30 $\times 13-15 \mu \mathrm{m})$ according to van Brummelen's pattern II. Clusters becoming longitudinally contracted with ascospores disposed according to pattern IIIa. Gelatinous perisporium not observed.

Notes - In sect. Eriobolus Sacc. (ascomata whitish or pigmented but never yellow, excipulum often with an intercellular pigment, paraphyses not containing yellow pigments, ascospores differently disposed from van Brummelen's pattern I), S. obscurus is characterised by brownish, pulvinate to discoidal ascomata, excipulum with a purple-brown intercellular pigment, warted ascospores firmly attached to each other and arranged in clusters according to van Brummelen's pattern II, spore clusters contracting at maturity. 


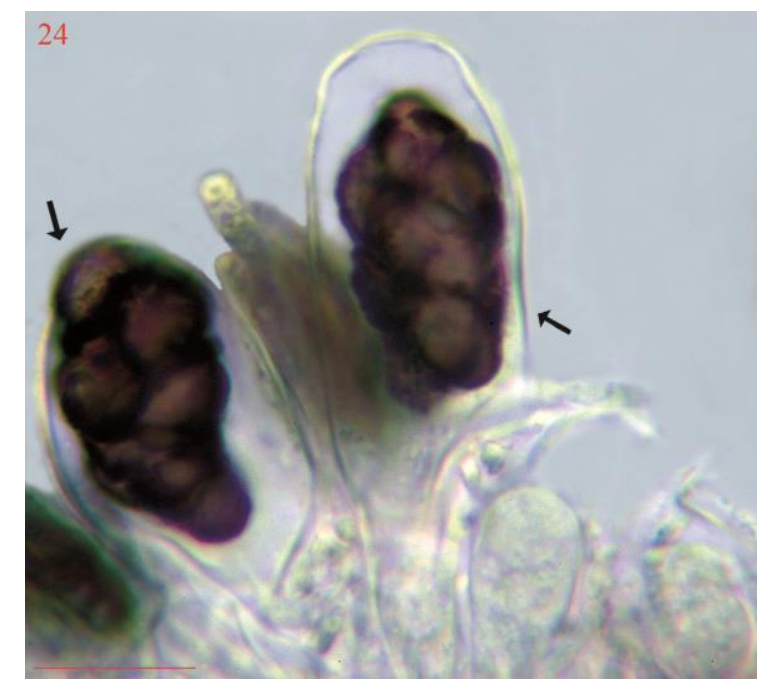

Fig 24 - Saccobolus obscurus. Mature asci (arrows) with spore clusters. - Bar $=15 \mu \mathrm{m}$.

The spore size of my first Italian collection of S. obscurus (CLSM 005.12) fully matches the protologue (Cooke 1876) but is somewhat smaller than that reported by van Brummelen (1967) after examining the type material (Cooke 1876) and some old European and extraeuropean collections. The spore shape of my collection is also very similar to that described by Heimerl (1889) and Korf \& Zhuang (1991), or drawn by van Brummelen (1967). On this basis we conceive S. obscurus as a taxon with ovoidal or ellipsoidal to broadly fusiform (average length/width ratio < 1.70), sometimes only slightly inequilateral ascospores, quite different to the ascospores of several European (Larsen 1970, Aas 1978a, Dennis 1981, Barrasa Gonzales 1985, van Vooren \& Meyer 2010) and Asian (Raitviir \& Prokhorov 1988) collections, which were described and/or drawn as elongated and inequilateral. According to the protologue (van Brummelen 1967), and in my experience, comparatively narrower (a larger length/width ratio) and more inequilateral ascospores are typical of $S$. verrucisporus Brumm., which also differs from $S$. obscurus in having smaller, whitish, cylindrical or subglobose ascomata, a pale violet intercellular pigment, and spore clusters not contracting at maturity. Like van Brummelen (1967), I have noticed in S. obscurus an episporium resembling $S$. verrucisporus, i.e. with both fine and coarse warts, and spore clusters without gelatinous sheaths. Others (Larsen 1970, Barrasa Gonzales 1985, Raitviir \& Prokhorov 1988), on the contrary, described S. obscurus with spore clusters having one unilateral or two polar sheaths, and only fine spore warts (van Vooren \& Meyer 2010), the latter stated to be quite different (Dissing et al. 2000) from the coarse warts of S. verrucisporus .

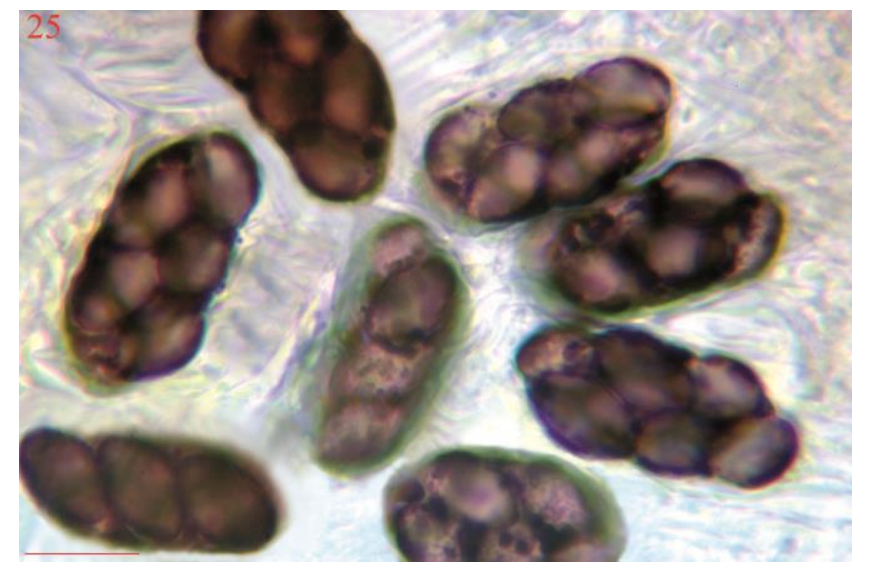

Fig 25 - Saccobolus obscurus. Spore clusters with ascospores in different stages of maturity. - Bar $=13 \mu \mathrm{m}$. 
The second Italian collection of S. obscurus (CLSM 005.12 bis) has an excipulum with slightly paler intercellular pigments, and somewhat longer ascospores (up to $16 \mu \mathrm{m}, 14.5 \mu \mathrm{m}$ on average) with more isolated and finer warts and an unilateral gelatinous sheath.

Also other species in sect. Eriobolus have warted ascospores, but S. tuberculatus Aas has cylindric and whitish ascomata with a pale rose intercellular pigment, and spore clusters not contracted at maturity (Aas 1978b), S. thaxteri Brumm. has somewhat smaller ascospores than $S$. obscurus and no excipular or hymenial pigment, and S. pampeanus Dokm., in my opinion the closest relative to $S$. obscurus, has an excipulum of textura globulosa-angularis and spore clusters not contracting at maturity (Dokmetzian et al. 1986).

S. obscurus is fairly infrequent but widespread. It has been recorded both from vegetable material (Cooke 1876, van Brummelen 1967, Larsen 1970, Dennis 1981 van Vooren \& Meyer 2010) and dung of several herbivores, occasionally of omnivores (Heimerl 1889). All the other species in this group are exclusively coprophilous. In my experience $S$. verrucisporus has a lower substrate tolerance than $S$. obscurus, as my Italian collections are usually from cervine droppings (91\%), with a $23 \%$ frequency on this type of dung in culture (Doveri 2011). Records from deer dung (van Brummelen 1967, Larsen 1970, Häffner 1986, Richardson 2001b) are prevalent (28\%) elsewhere, but the frequency is lower than in Italy. S. thaxteri has been recorded from goat, squirrel (van Brummelen 1967) and rabbit (Otani \& Kanzawa 1970, Piontelli et al. 2006) dung, $S$. pampeanus from horse (Dokmetzian et al. 1986, Ranalli \& Mercuri 1995), S. tuberculatus from sheep, but the real substrate choice of the last three species cannot be stated for now, as they are infrequent and rarely reported.

Update on collections of coprophilous species from Italy (for synonyms, see van Brummelen 1967 and Doveri 2004)

\section{Ascobolus aglaosporus}

Figs $1-10$

Material examined - 1) LIVORNO, Quercianella, $50 \mathrm{~m}$, three supericial, gregarious specimens on fallow deer dung in d.c., F. Doveri, 22.11.12, 284.3-Rosignano Marittimo, CLSM 008.12. 2) LIVORNO, Quercianella, 0 m, on fallow deer dung in d.c., F. Doveri, 19.12.12, 284.3Rosignano Marittimo, CLSM 008.12 bis.

TOTAL 2: fallow deer 2.

Ascobolus albidus H. Crouan \& P. Crouan, Ann. Sci. Nat. (Bot.) 4 (10): 193, 1858.

Figs 26-31

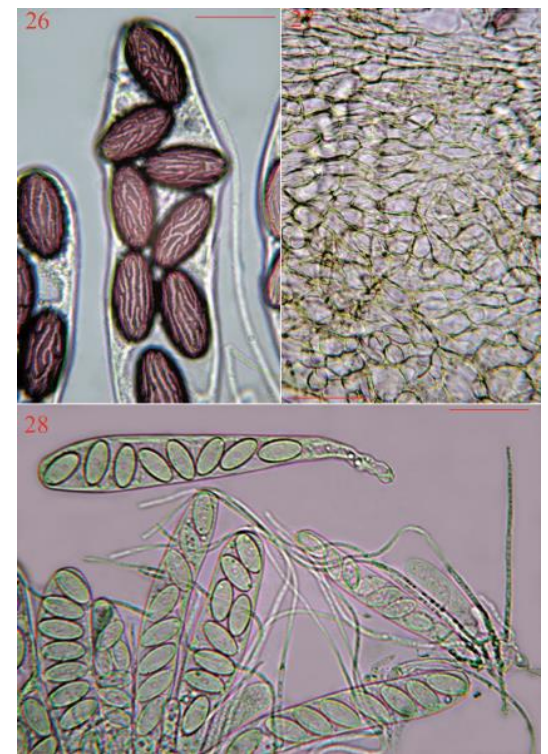

Figs 26-28 - Ascobolus albidus. 26 Asci with ascospores. 27 Detail of middle and upper ectal excipulum. 28 Immature asci with ascospores and filiform paraphyses. - Bars $26=20 \mu \mathrm{m} .27-28=25 \mu \mathrm{m}$. 
Material examined - 1) PISA, Calambrone stables, $0 \mathrm{~m}$, about ten specimens, some of which crowded, on horse dung in d.c, F. Doveri, 2.6.95, 272.2-Marina di Pisa, MCVE 480. 2) PISA, Calambrone stables, $0 \mathrm{~m}$, on horse dung in d.c., F. Doveri, 23.11.95, CLSM 01395. 3) ROVIGO, Porto Caleri, 0 m, on horse dung in d.c., F. Doveri, 5.97, 169.2-Contarina, CLSM 01395 ter. 4) FERRARA, S. Giustina wood (Mesola), $0 \mathrm{~m}$, on horse dung in d.c., F. Bersan \& G. Visentin, 5.97., 187.1.-Mesola, CLSM 01395 quater. 5) GROSSETO, Principina Terra, $0 \mathrm{~m}$, on horse dung in d.c., F. Doveri, 2.4.98, 331.3-Alberesese, CLSM 01395 penta. 6) FERRARA, Mesola, 0 m, on deer dung, A. Bizzi \& G. Zecchin, 17.4.99, 187.1-Mesola, CLSM 01395 esa. 7) COSENZA, S. Demetrio Corone, $800 \mathrm{~m}$, on goat dung in d.c., C. Lavorato, 10.5.99, 552.4-S. Demetrio Corone, CLSM 01395 epta. 8) LIVORNO, Corbolone, on horse dung, G. Cacialli \& F. Doveri, 14.4.00, 284.4-Collesalvetti, CLSM 01395 octo. 9) GENOVA, Portofino Park, on wild goat dung in d.c., L. Levorato, 30.4.01, 231.4-Recco, CLSM 01395 ena. 10) COSENZA, Celico, $1200 \mathrm{~m}$, on horse dung in d.c., C. Lavorato, 4.9.01, 560.3-Spezzano della Sila, CLSM 01395 deca. 11) VENEZIA, Alberoni, $0 \mathrm{~m}$, on horse dung in d.c., A. Bizzi, 23.2.02, 148.1-Alberoni, CLSM 01395-XI. 12) COSENZA, Acri, $1000 \mathrm{~m}$, on wild pig dung, C. Lavorato, 10.5.02, 552.3-Acri, CLSM 01395-XII. 13) LIVORNO, Palazzi di Cecina, $0 \mathrm{~m}$, on cattle dung, F. Doveri, 30.4.06, 294.1-Cecina, CLSM 01395-XIII. 14) VERONA, Soave, $350 \mathrm{~m}$, on horse dung in d.c., A. Bizzi, 17.4.06, 124.2-Soave, CLSM 01395-XIV. 15) VICENZA, Valdagno, Zovo pass, $600 \mathrm{~m}$, on horse dung in d.c., A. Bizzi, 18.5.06, 102.2-Valdagno, CLSM 01395-XV. 16) VERONA, Erbezzo, Lessinia park, $1570 \mathrm{~m}$, on horse dung in d.c., A. Bizzi, 1.6.06, 102.3-Bosco Chiesanuova, CLSM 01395-XVI. 17) PISA, San Miniato Basso, 0 m, on horse dung in d.c., F. Doveri, 13.4.09, 274.2-San Miniato, CLSM 01395XVII. 18) GROSSETO, Monterotondo Marittimo, boraciferous lake, $250 \mathrm{~m}$, on horse dung in d.c., F. Doveri, 25.3.11, 306.4-Monterotondo Marittimo, CLSM 01395-XVIII.

TOTAL 18: horse 13; goat 2; cattle 1; deer 1; wild pig 1.

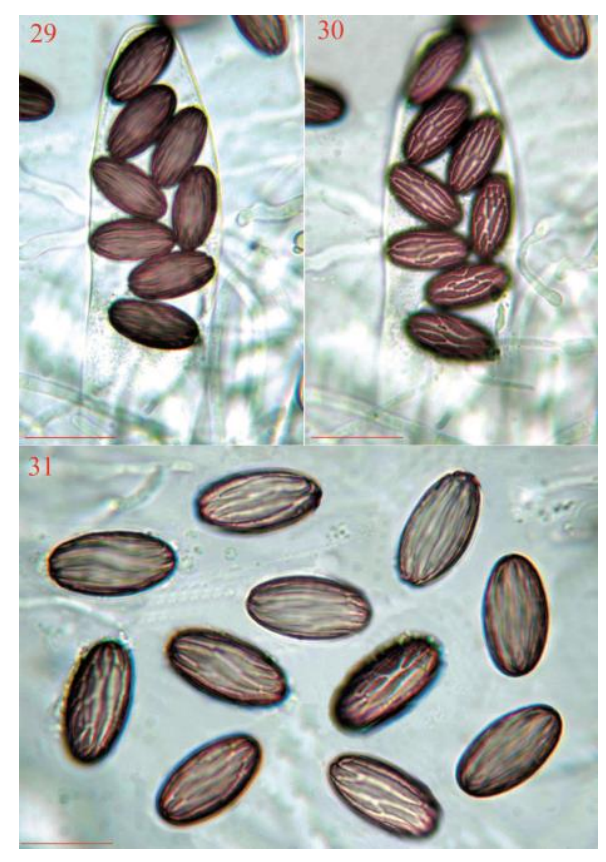

Figs 29-31 - Ascobolus albidus. 29-30 Ascus with ascospores in different focuses. 31 Free mature ascospores. - Bars 29-30 $=20 \mu \mathrm{m} .31=15 \mu \mathrm{m}$.

Ascobolus brassicae H. Crouan \& P. Crouan, Ann. Sci. Nat. (Bot.) 4 (7): 174, $1857 . \quad$ Figs 32-38

Material examined - 1) COSENZA, Fossiata-Sila National Park, $1400 \mathrm{~m}$, about ten gregarious, superficial specimens on wolf dung, C. Lavorato, 22.5.98, 560.1-Monte Volpintesta, CLSM 04798. 2) COSENZA, S. Demetrio Corone, $800 \mathrm{~m}$, on rat dung in d.c., C. Lavorato, 14.1.00, 552.4-S. Demetrio Corone, CLSM 04798 bis. 


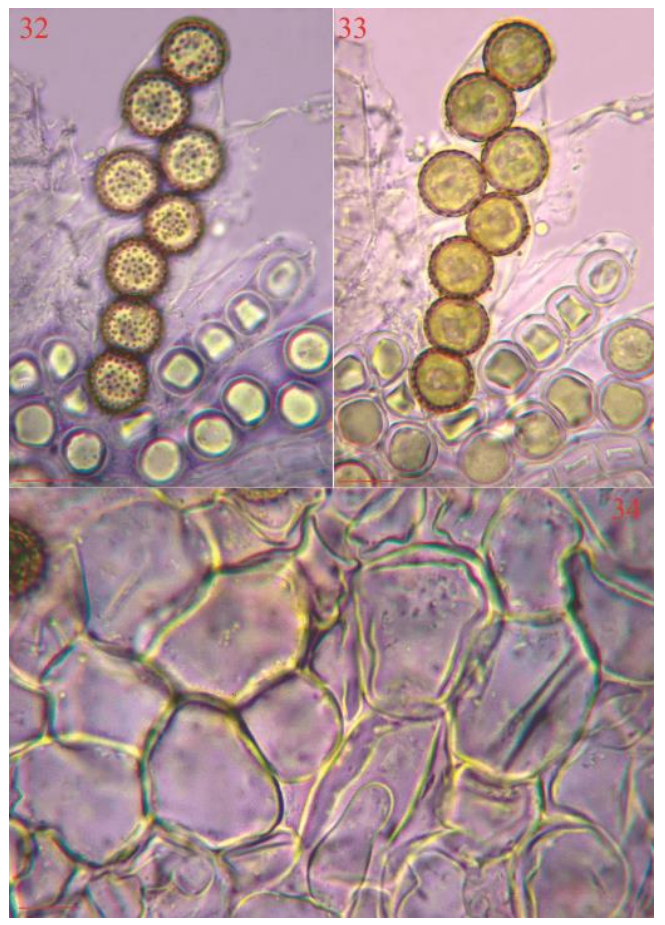

Figs 32-34 - Ascobolus brassicae. 32-33 Ascus with ascospores in different focuses. 34 Detail of ectal excipulum. - Bars $32-33=12 \mu \mathrm{m} .34=10 \mu \mathrm{m}$.

TOTAL 2: rat 1; wolf 1 .

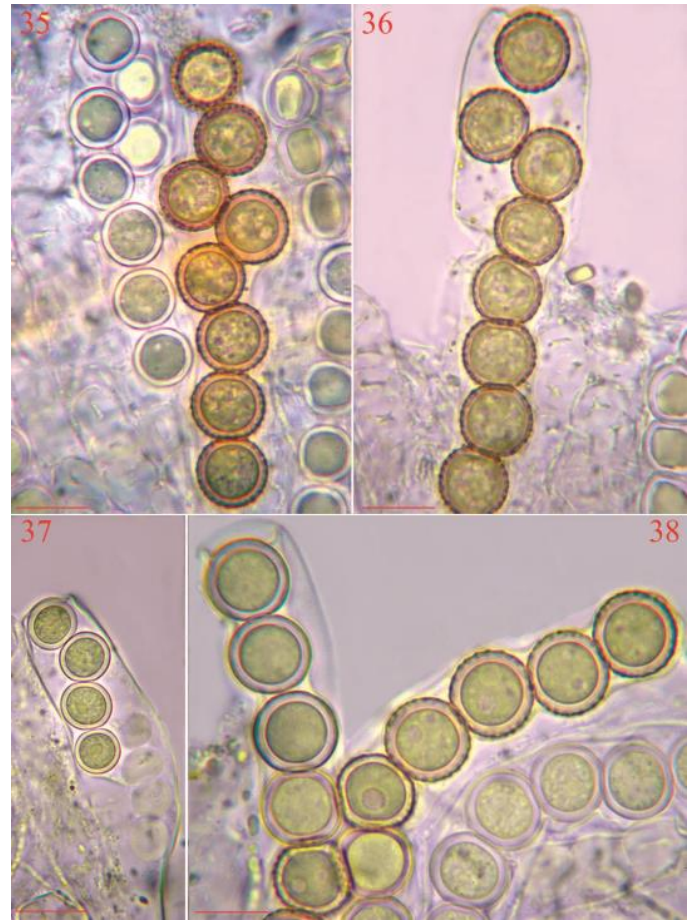

Figs 35-38 - Ascobolus brassicae. Asci with ascospores in different stages of maturity. - Bars 35$36=12 \mu \mathrm{m} .37=15 \mu \mathrm{m} .38=10 \mu \mathrm{m}$.

Ascobolus carletoni Boud., Trans. Br. Mycol. Soc. 4: 62, 1913.

Figs 39-42

Material examined - BELLUNO, Falcade, ?, about thirty gregarious specimens on tetraonid dung in d.c., E. Bizio, 15.8.02, 045.1-Cencenighe Agordino, CLSM 019.02. 


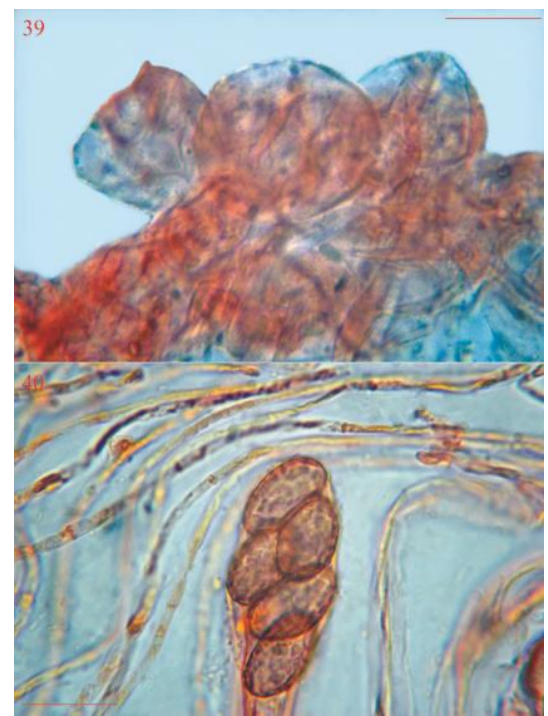

Figs 39-40 - Ascobolus carletoni. 39 Cells of the ectal excipulum. 40 Ascus with mature ascospores and overarching paraphyses. - Bars $39=20 \mu \mathrm{m} .40=12 \mu \mathrm{m}$.

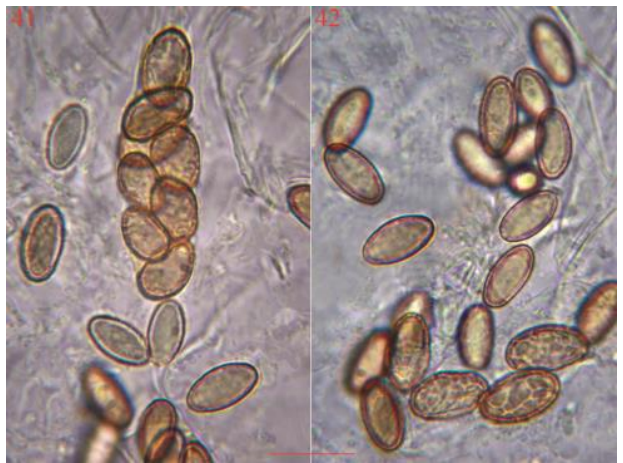

Figs 41-42 - Ascobolus carletoni. 41 Free ascospores and ascus with biseriate ascospores. 42 Ascospores in different stages of maturity. - Bar $=15 \mu \mathrm{m}$.

Ascobolus costantinii Rolland, Bull. Soc. Mycol. Fr. 4: 56, 1888.

Figs 43-47

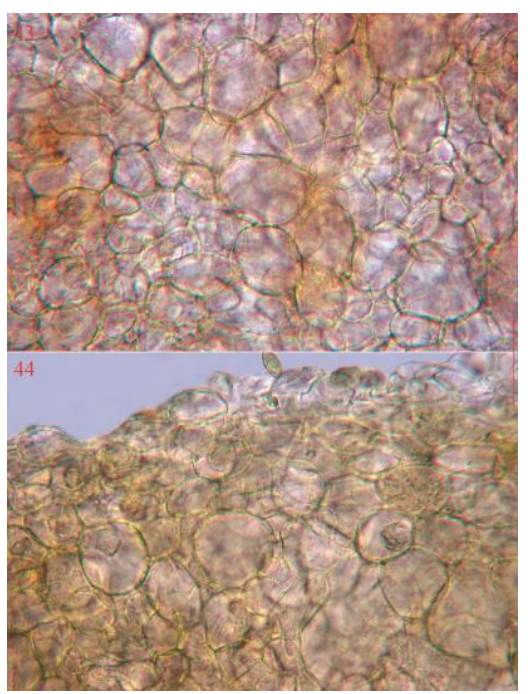

Figs 43-44 - Ascobolus costantini. 43 Detail of ectal excipulum. 44 Ectal excipulum at the margin. - Bar $=50 \mu \mathrm{m}$. 
Material examined - 1) ROVIGO, Porto Caleri, $0 \mathrm{~m}$, about thirty gregarious specimens on dung of an unidentified animal in d.c., F. Doveri, 5.97, 169.2-Contarina, MCVE 582. 2) LIVORNO, Quercianella, $0 \mathrm{~m}$, on fallow deer dung in d.c., F. Doveri, 19.12.12, 284.3-Rosignano Marittimo, CLSM 00997 bis.

TOTAL 2: deer 1; unidentified animal 1.

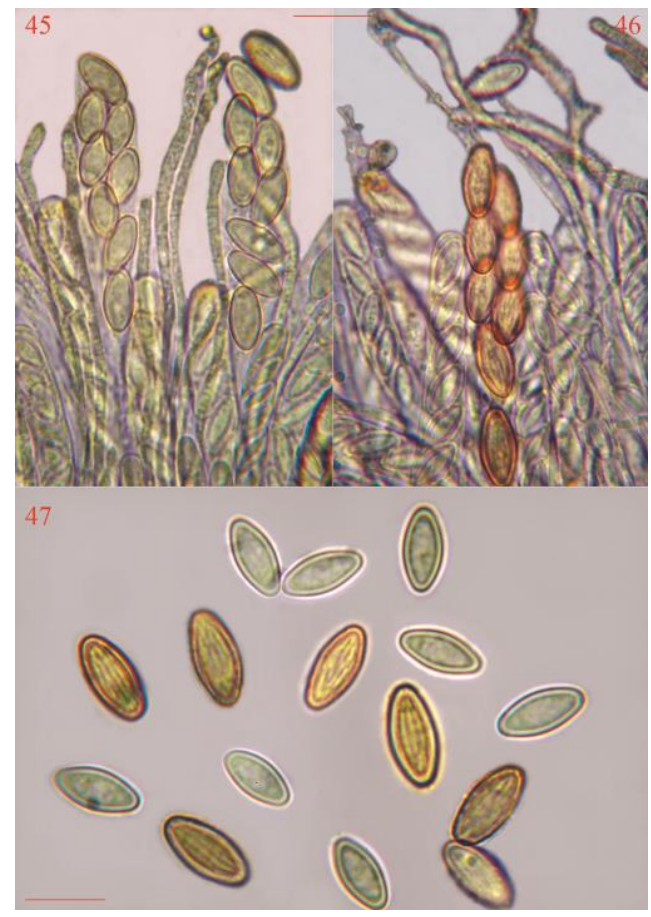

Figs 45-47 - Ascobolus costantini. 45-46 Asci with biseriate ascospores in different stages of maturity, and exceeding paraphyses. 47 Ascospores in different stages. - Bars $45-46=15 \mu \mathrm{m} .47=$ $12 \mu \mathrm{m}$.

Ascobolus crenulatus P.Karst., Fungi Fenn. Exs.: 763, 1868.

Figs 48-51

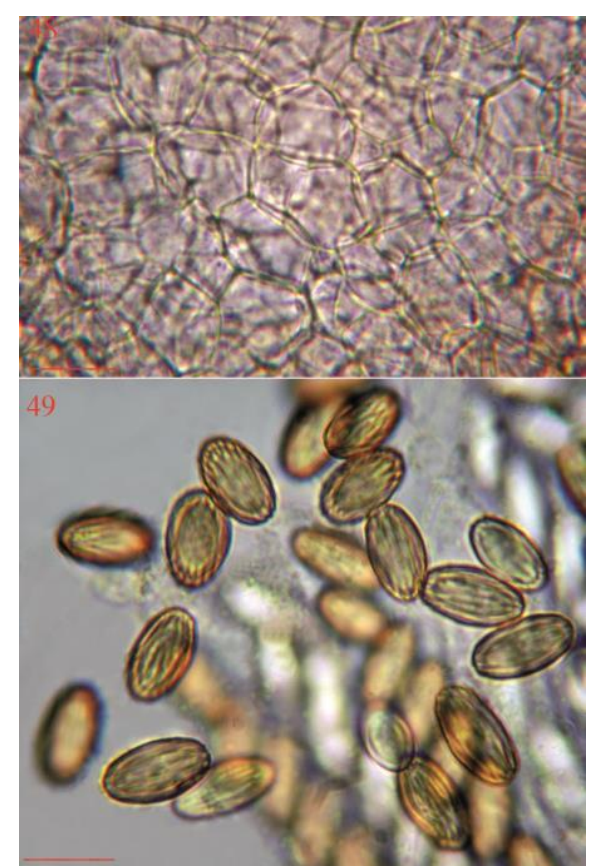

Figs 48-49 - Ascobolus crenulatus. 48 Detail of ectal excipulum. 49 Free mature ascospores. Bars $48=40 \mu \mathrm{m} .49=10 \mu \mathrm{m}$. 
Material examined - 1) BRESCIA, Collebeato, $300 \mathrm{~m}$, dozens of gregarious, superficial specimens, many of which crowded, on dung of an unidentified herbivore, G. Medardi, 5.92, 121.1-Brescia, MCVE-ERB2 10752. 2) ROVIGO, Albarella Isle, $0 \mathrm{~m}$, on dung of an unidentified carnivore in d.c., G. Robich, 14.5.99, 170.3-Porto Levante, CLSM 02896 ter. 3) MODENA, Zocca, $750 \mathrm{~m}$, on hedgehog dung in d.c., L. Piccioli, 12.99, 237.4-Savigno, CLSM 02896 quater. 4) COSENZA, S. Demetrio Corone, $800 \mathrm{~m}$, on rat dung in d.c., C. Lavorato, 14.1.00, 552.4-S. Demetrio Corone, CLSM 02896 penta. 5) MILANO, Triuggio, on wild rabbit dung in d.c., F. Doveri, 25.5.00, 096.2-Seregno, CLSM 02896 esa. 6) RAVENNA, Lido di Classe, 0 m, on wild rabbit dung in d.c., F. Doveri, 6.4.01, 241.3-Cervia, CLSM 02896 epta. 7) COSENZA, S. Demetrio Corone, $800 \mathrm{~m}$, on weasel dung in d.c., C. Lavorato, 16.8.01, 552.4-S. Demetrio Corone, CLSM 02896 octo. 8) ROMA, Castel Porziano, 0 m, on wild pig dung in d.c., G. Robich, 3.12.01, 386.1Lido di Ostia, CLSM 02896 ena. 9) VICENZA, Nagarole, Mount Faldo, 700 m, on hare dung in d.c., A. Bizzi, 20.4.04, 102.2-Valdagno, CLSM 02896 deca. 10) PISA, La Sassa, 350 m, on roe deer dung in d.c., F. Doveri, 15.10.12, 295.3-Sassa, CLSM 02896-XI.

TOTAL 10: wild rabbit 2; hare 1; hedgehog 1; rat 1; roe deer 1 ; weasel 1 ; wild pig 1 ; unidentified carnivore 1; unidentified herbivore 1 .

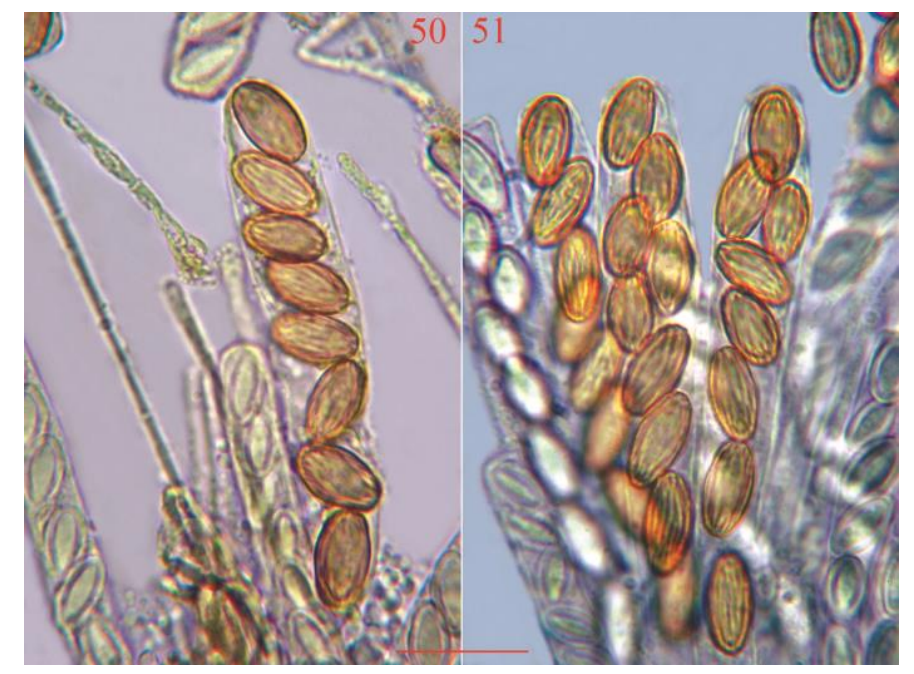

Figs 50-51 - Ascobolus crenulatus. 50 Ascus with mature, uniseriate ascospores, surrounded by paraphyses. 51 Asci with mature, biseriate ascospores. - Bar $=20 \mu \mathrm{m}$.

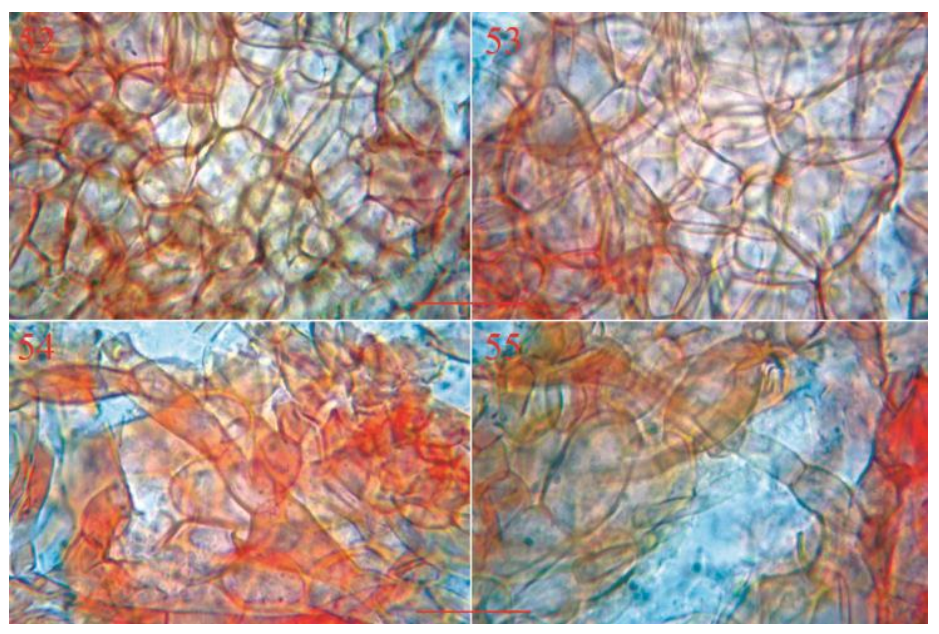

Figs 52-55 - Ascobolus elegans. Details of ectal excipulum. - Bars $=20 \mu \mathrm{m}$. 
Material examined - 1) GROSSETO, "La Principina" farm (Principina Terra resort), $0 \mathrm{~m}$, about one hundred gregarious, sometimes clustered, superficial specimens on horse dung in d.c., F. Doveri, 14.3.96, 331.3-Alberesese, MCVE 574. 2) FERRARA, S. Giustina wood (Mesola), 0 m, on horse dung in d.c., 5.97, F. Bersan \& G. Visentin, 187.1-Mesola, CLSM 01496 bis. 3) GORIZIA, Cona island, $0 \mathrm{~m}$, on horse dung in d.c., F. Bersan, 8.97, 109.1-Duino, CLSM 01496 ter. 4) GROSSETO, Principina Terra, $0 \mathrm{~m}$, on horse dung in d.c., F. Doveri, 2.4.98, 331.3-Alberese, CLSM 01496 quater. 5) MILANO, Monza park, 150 m, on horse dung in d.c., F. Doveri, 26.5.00, 118.1-Monza, CLSM 01496 penta.

TOTAL 5: horse 5.

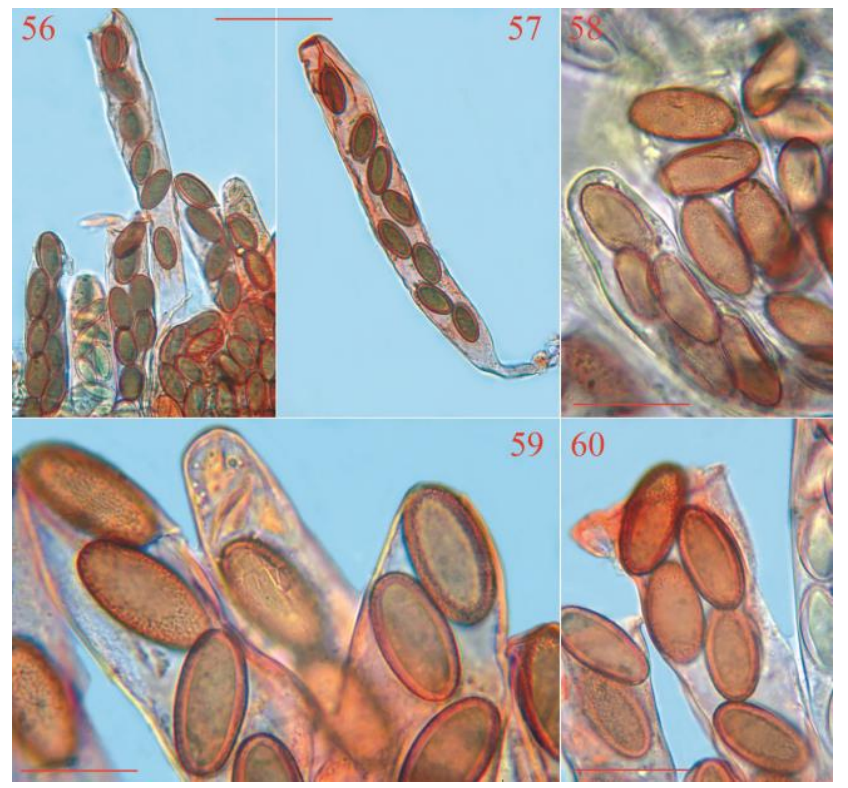

Figs 56-60 - Ascobolus elegans. Asci with mature ascospores. - Bars 56-57 $=80 \mu \mathrm{m} .58=35 \mu \mathrm{m}$. $59=25 \mu \mathrm{m} .60=30 \mu \mathrm{m}$.

Ascobolus furfuraceus Pers., Neues Mag. Bot. 1: 115, 1794.

Figs 61-67

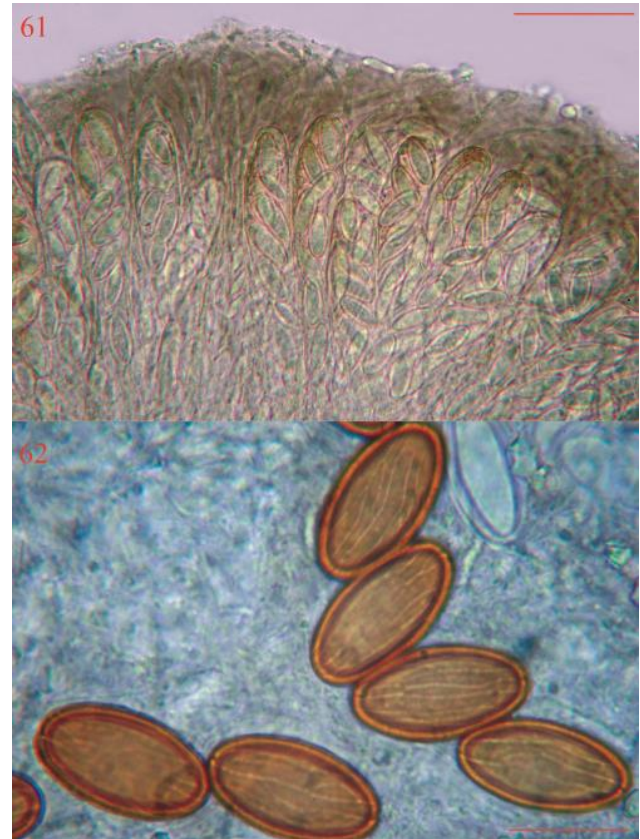

Figs 61-62 - Ascobolus furfuraceus. 61 Detail of humenial surface with paraphyses embedded in a gelatinous material. 62 Ascospores. - Bars $61=40 \mu \mathrm{m} .62=20 \mu \mathrm{m}$. 
Material examined - 1) LIVORNO: Botro delle Fontanelle, $200 \mathrm{~m}$, dozens of gregarious, sometimes crowded specimens, on cattle dung, F. Doveri, 29.10.92, 284.4-Collesalvetti, AMBLivorno 12692. 2) LIVORNO: Botro delle Fontanelle, $200 \mathrm{~m}$, on cattle duhg, F. Doveri, 29.8.93, 284.4-Collesalvetti, AMB-Livorno 12692 bis. 3) LIVORNO: Botro delle Fontanelle, $200 \mathrm{~m}$, on cattle dung, F. Doveri, 12.2.94, 284.4-Collesalvetti, AMB-Livorno 12692 ter. 4) UDINE, Pura Pass, $1450 \mathrm{~m}$, on marten dung, F. Bersan, 11.9.97, 031.3-Ampezzo, CLSM 12692 bis. 5) TRENTO, Panarotta refuge, $1800 \mathrm{~m}$, on cattle dung, A. De Vito, 25.9.97, 060.2-Pergine, CLSM 12692 ter. 6) TRENTO, Marzola refuge, $1800 \mathrm{~m}$, on roe deer dung in d.c., M. Floriani, 26.9.97, 060.2-Pergine, CLSM 12692 quater. 7) TREVISO, Scalon, 230 m, on sheep dung in d.c., E. Bizio, 6.6.97, 083.1Valdobbiadene, CLSM 12692 penta. 8) BERGAMO, Dossena, 900 m, on cattle dung, A. De Vito, 7.9.97, 077.3-San Pellegrino Terme, CLSM 12692 esa. 9) GROSSETO, Principina Terra, 0 m, on cattle dung in d.c., F. Doveri, 2.4.98, 331.3-Alberesese, CLSM 12692 epta. 10) PORDENONE, Frisanco, 500 m, 18.6.98, on deer dung in d.c., G. Zecchin, 18.6.98, 065.4-Maniago, CLSM 12692 octo. 11) VICENZA, Nogarole Vicentino (Mount Faldo), 600 m, on horse dung in d.c., A. Bizzi, 1.9.99, 124.1-Arzignano, CLSM 12692-ena. 12) COSENZA, S. Demetrio Corone, $800 \mathrm{~m}$, on goat dung in d.c., C. Lavorato, 10.5.99, 552.4-S. Demetrio Corone, CLSM 12692 deca. 13) MILANO, Monza Park, 150 m, on horse dung in d.c., F. Doveri, 26.5.00, 118.1-Monza, CLSM 12692-XII. 14) VERONA, Podesteria, $1500 \mathrm{~m}$, on cattle dung in d.c., M. Zugna, 5.10.00, 102.3-Bosco Chiesanuova, CLSM 12692-XIII. 15) AOSTA, Salati pass, 3000 m, on rock goat dung in d.c., L. Levorato, 23.8.00, 071.3-Gressoney la Trinité, CLSM 12692-XIV. 16) AOSTA, Bettaforca, 2800 $\mathrm{m}$, on rock goat dung in d.c., L. Levorato, 26.8.00, 071.3-Gressoney la Trinité, CLSM 12692-XV. 17) LECCE, Vanze, $10 \mathrm{~m}$, on horse dung in d.c., V. Sciurti \& F. Doveri, 24.11.01, 513.4-S. Foca, CLSM 12692-XVI. 18) TREVISO, Borso-loc. Mount Grappa, Campo Croce, $1200 \mathrm{~m}$, on roe deer (?) dung in d.c., A. Bizzi, 10.8.01, 083.3-Monte Grappa, CLSM 12692-XVII. 19) COSENZA, AcriContrada Mischinella, $1100 \mathrm{~m}$, on wild pig dung in d.c., C. Lavorato, 19.10.01, 552.3-Acri, CLSM 12692-XVIII. 20) COSENZA, Cozzo del Pesco, $1200 \mathrm{~m}$, on cattle dung in d.c., C. Lavorato, 9.9.01, 561.4-Savelli, CLSM 12692-XIX. 21) LECCO, Concenedo, $920 \mathrm{~m}$, on cattle dung in d.c., F. Doveri, 6.6.03, 076.4-Barzio, CLSM 12692-XX. 22) REGGIO CALABRIA, Tazza, $800 \mathrm{~m}$, on cattle dung, F. Doveri, 17.10.03, 602.3-Motta San Giovanni, CLSM 12692-XXI. 23) VICENZA, S. Zeno park, ? m, on roe deer dung in d.c., A. Bizzi, 6.11.03, 103.4-Schio, CLSM 12692-XXII. 24) LIVORNO, Palazzi di Cecina, 0 m, on cattle dung, F. Doveri, 30.4.06, 294.1-Cecina, CLSM 12692XXIV. 25) TRENTO, Dimaro, Malga di Sadron, 1450 m, on cattle dung, F. Doveri, 8.9.07, 042.1Malé, CLSM 12692-XXV. 26) PISA, San Miniato Basso, 0 m, on horse dung, F. Doveri, 13.4.09, 274.2-San Miniato, CLSM 12692-XXVI.

TOTAL 26: cattle 12; horse 4; roe deer 3 ; rock goat 2 ; deer 1 ; goat 1 ; marten 1 ; sheep 1 ; wild pig 1.

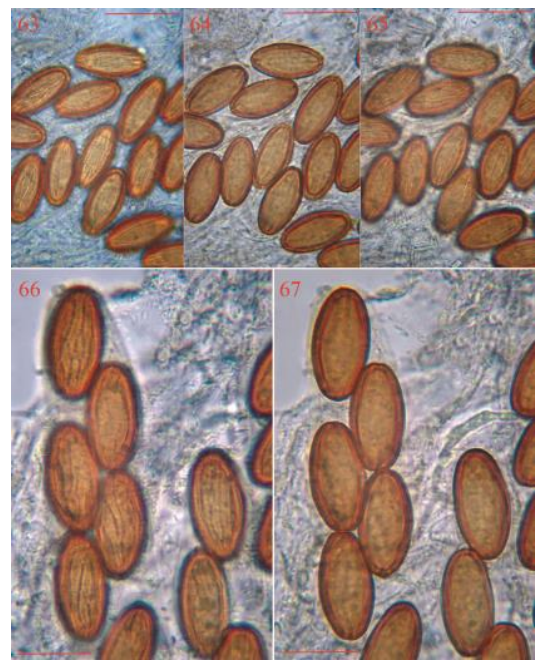

Figs 63-67 - Ascobolus furfuraceus. Ascospores in different focuses. - Bars $63-65=30 \mu \mathrm{m} .66-67$ $=15 \mu \mathrm{m}$. 


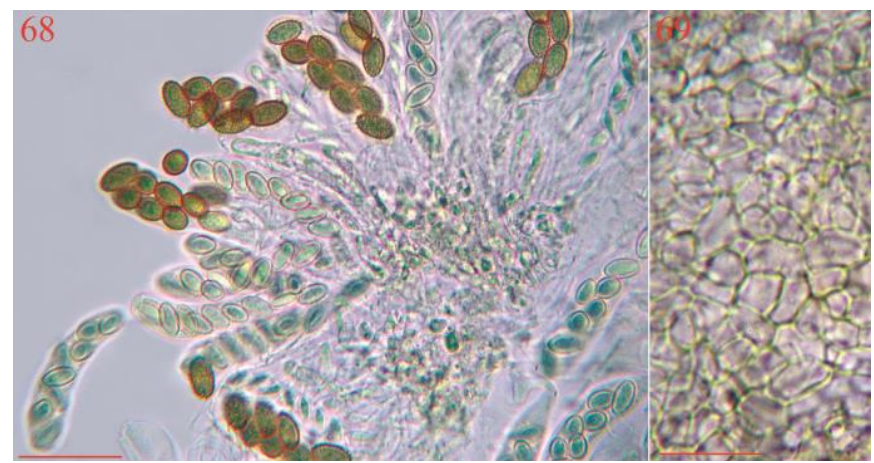

Figs 68-69-Ascobolus hawaiiensis. 68 Detail of humenial surface. 69 Detail of ectal excipulum. Bars $68=50 \mu \mathrm{m} .69=25 \mu \mathrm{m}$.

Material examined - 1) FERRARA, S. Giustina wood (Mesola), $0 \mathrm{~m}$, dozens of gregarious, superficial specimens on horse dung in d.c., F. Bersan \& G. Visentin, 5.97, 187.1-Mesola, MCVE 581. 2) VICENZA, Grancona, Mount Caldiero, $230 \mathrm{~m}$, on sheep dung in d.c., A. Bizzi, 10.00, 125.3-Montebello, CLSM 01097 bis. 3) VICENZA, Sarego, Mount Roccolo, $250 \mathrm{~m}$, on sheep dung in d.c., A. Bizzi, 10.00, 125.3-Montebello, CLSM 01097 ter. 4) POTENZA, Sasso di Castalda, $1000 \mathrm{~m}$, on sheep dung in d.c., A. Bizzi, 10.11.00, CLSM 01097 quater.

TOTAL 4: sheep 3; horse 1.

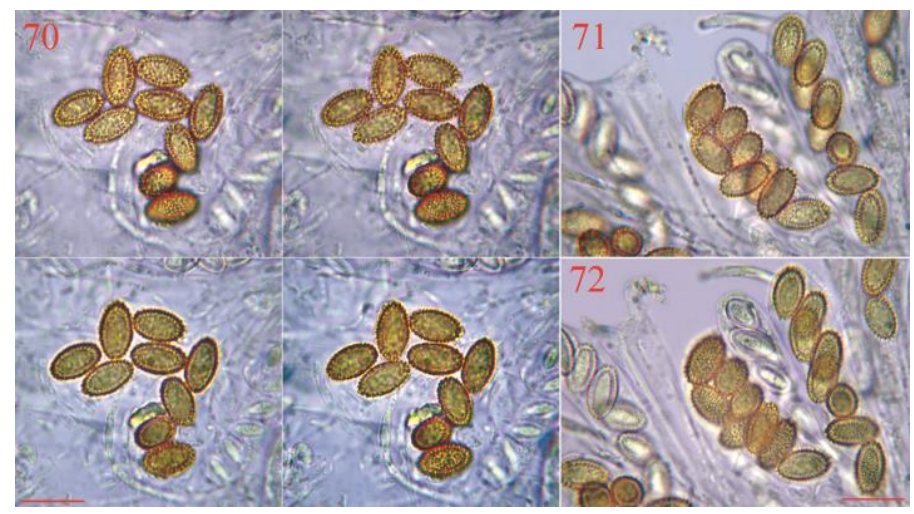

Figs 70-72 - Ascobolus hawaiiensis. 70 Free mature ascospores in different focuses. 71-72 Asci with biseriate ascospores in different focuses. - Bars 70-72 $=20 \mu \mathrm{m}$.

Ascobolus immersus Pers. ex Pers., Mycol. Eur. 1: 341, 1822.

Figs 73-78

Material examined - 1) TRENTO, Malga di Fondo, $1400 \mathrm{~m}$, thousands of gregarious specimens, often crowded, superficial or partly immersed in cattle dung, F. Doveri, 17.9.94, 026.3Fondo, CCD-Livorno 00594. 2) PISA, Calambrone, 0 m, on horse dung, F. Doveri, 10.96, 272.2Marina di Pisa, CCD-Livorno 00594 bis. 3) PISA, Calambrone, on horse dung, F. Doveri, 18.2.97, 272.2-Marina di Pisa, CCD-Livorno 00594 ter. 4) TREVISO, Scalon, $200 \mathrm{~m}$, on sheep dung in d.c., E. Bizio, 6.6.97, 083.1-Valdobbiadene, CLSM 00594 quater. 5) BELLUNO, Forcella Negher, 2300 m, on marmot dung in d.c., E. Bizio, 2.9.97, 045.1-Cencenighe Agordino, CLSM 00594 penta. 6) TRENTO, Laghestèl, 1000 m, on horse dung in d.c., F. Doveri, 25.9.97, 060.1-Cembra, CLSM 00594 esa. 7) BERGAMO, Dossena, 1000 m, on cattle dung in d.c., A. De Vito, 21.8.97, 077.3-San Pellegrino Terme, CLSM 00594 epta. 8) GROSSETO, Principina Terra, 0 m, on horse dung in d.c., F. Doveri, 2.4.98, 331.3-Alberesese, CLSM 00594 octo. 9) GROSSETO, Principina Terra, 0 m, on rabbit dung in d.c., F. Doveri, 2.4.98, 331.3-Alberesese, CLSM 00594 ena. 10) COSENZA, Fossiata, $1400 \mathrm{~m}$, on wolf dung in d.c., C. Lavorato, 22.5.98, 560.1-Monte Volpintesta, 00594 deca. 


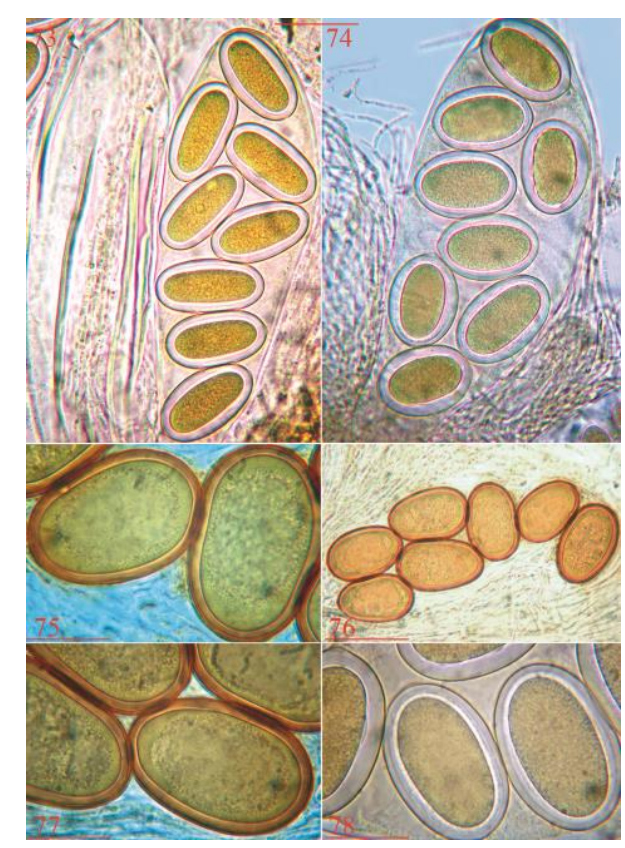

Figs 73-78 - Ascobolus immersus. 73 Ascus with immature, biseriate ascospores. 74 Ascus with ascospores, surrounded by paraphyses. 75-78 Ascospores in different stages of maturity. - Bars 73$74=50 \mu \mathrm{m} .75,77-78=25 \mu \mathrm{m} .76=60 \mu \mathrm{m}$.

11) COSENZA, Fossiata, $1400 \mathrm{~m}$, on fox dung in d.c., C. Lavorato, 22.5.98, 560.1-Monte Volpintesta, 00594-XI. 12) COSENZA, Orsomarso, $1300 \mathrm{~m}$, on donkey dung in d.c., C. Lavorato, 17.5.98, 533.2-Mormanno, CLSM 00594-XII. 13) VICENZA, Recoaro, $1000 \mathrm{~m}$, on sheep dung in d.c., A. Bizzi, 10.9.98, 102.1-Recoaro Terme, CLSM 00594-XIII. 14) MESSINA, Novara di Sicilia, on deer dung, G. Robich, 11.11.98, 613.4-Francavilla di Sicilia, CLSM 00594-XIV. 15) MESSINA, Randazzo, 1800 m, on sheep dung, A. Bizzi, 612.2-Randazzo, CLSM 00594-XV. 16) COSENZA, Rossano, 700 m, on wild pig dung in d.c., C. Lavorato, 22.12.98, 552.1-Corigliano Calabro, CLSM 00594-XVI. 17) COSENZA, Corigliano Calabro, $0 \mathrm{~m}$, on sheep dung, C. Lavorato, 22.12.98, 552.1-Corigliano Calabro, CLSM 00594-XVII. 18) BELLUNO, Forcella Negher, 2300 m, on rock goat dung in d.c., E. Bizio, 6.9.99, 045.1-Cencenighe Agordino, CLSM 00594-XVIII. 19) TRENTO, Malga Giumella (Pejo Valley), $1700 \mathrm{~m}$, on goat dung, M. Zugna, 8.9.99, 041.1-Corno dei Tre Signori, CLSM 00594-XIX. 20) TRENTO, Forcella Juribrutto, $2400 \mathrm{~m}$, on sheep dung in d.c., E. Bizio, 28.7.99, 045.4-Soraga di Fassa, CLSM 00594-XX. 21) AOSTA, Salati Pass, 2970 m, on rock goat dung in d.c., L. Levorato, 21.8.99., 071.3-Gressoney la Trinité, CLSM 00594-XXI. 22) VICENZA, Lugo, 350 m, on horse dung, A. Bizzi, 29.10.99, 103.1-Marostica, CLSM 00594-XXII. 23) VERONA, Roncà (Mount Calvarina), 600 m, on sheep dung in d.c., A. Bizzi, 25.9.99, 124.1Arzignano, CLSM 00594-XXIII. 24) NUORO, Cardedu (loc. Perda Pera), $0 \mathrm{~m}$, on sheep dung in d.c., A. Bizzi, 7.11.99, 541.1-Jerzu, CLSM 00594-XXIV. 25) NUORO, Tertenia (loc. Sucrabiolus), $150 \mathrm{~m}$, on sheep dung, A. Bizzi, 7.11.99, 541.2-Tertenia, CLSM 00594-XXV. 26) CAGLIARI, Villasimíus, $100 \mathrm{~m}$, on sheep dung in d.c., A. Bizzi, 9.11.99, 567.1-Villasimíus, CLSM 00594XXVI. 27) PISA, S. Rossore Park, $0 \mathrm{~m}$, on fallow deer dung in d.c., F. Doveri, 5.3.00, 272.1Migliarino, CLSM 00594-XXVII. 28) LIVORNO, Corbolone, $150 \mathrm{~m}$, on horse dung in d.c., G. Cacialli \& F. Doveri, 14.4.00, 284.4-Collesalvetti, CLSM 00594-XXIX. 29) VERCELLI, Mount Rosa (Gabiet Lake), 2500 m, on rock goat dung in d.c., G. Robich, 26.8.00, 071.2-Alagna Valsesia, CLSM 00594-XXX. 30) POTENZA, Sasso di Castalda, 1000 m, on sheep dung, A. Bizzi, 10.11.00, 488.2-Brienza, CLSM 00594-XXXI. 31) BELLUNO, Lozzo (Pian dei Buoi), $1800 \mathrm{~m}$, on hare dung in d.c., L. Levorato, 10.9.00, 030.4-Auronzo di Cadore, CLSM 00594-XXXII. 32) POTENZA, Monticchio, $600 \mathrm{~m}$, on sheep dung in d.c., A. Bizzi, 16.11.00, 451.1-Melfi, 00594-XXXIII. 33) BELLUNO, Lozzo (Pian dei Buoi), 1800 m, on roe deer dung in d.c., L. Levorato, 10.9.00, 030.4- 
Auronzo di Cadore, CLSM 00594-XXXIV. 34) AOSTA, Rio Endre, 2800 m, on rock goat dung in d.c., L. Levorato, 24.8.00, 071.3-Gressoney la Trinité, CLSM 00594-XXXV. 35) AOSTA, Rio Endre, $2800 \mathrm{~m}$, on marmot dung in d.c., L. Levorato, 24.8.00, 071.3-Gressoney la Trinité, CLSM 00594-XXXVI. 36) AOSTA, Salati Pass, 3000 m, on rock goat dung in d.c., L. Levorato, 23.8.00, 071.3-Gressoney la Trinité, CLSM 00594-XXXVII. 37) COSENZA, Rossano, $900 \mathrm{~m}$, on cattle dung in d.c., C. Lavorato, 22.9.00, 552.1-Corigliano Calabro, CLSM 00594-XXXVIII. 38) COSENZA, Rossano, $900 \mathrm{~m}$, on sheep dung in d.c., C. Lavorato, 22.9.00, 552.1-Corigliano Calabro, CLSM 00594-XXXIX. 39) COSENZA, Longobucco (loc. Pettinascura), $1580 \mathrm{~m}$, on sheep dung in d.c., C. Lavorato, 2.4.01, 560.1-Monte Volpintesta, CLSM 00594-XL. 40) COSENZA, Acri (loc. S. Zagaria), $800 \mathrm{~m}$, on donkey dung in d.c., C. Lavorato, 25.2.01, 552.3-Acri, CLSM 00594-XLI. 41) COSENZA, S. Giovanni in Fiore, $1600 \mathrm{~m}$, on roe deer dung in d.c., C. Lavorato, 26.2.01, 560.1-Monte Volpintesta, CLSM 00594-XLII. 42) COSENZA, Acri (Manche di Greca), $1100 \mathrm{~m}$, on goat dung in d.c., C. Lavorato, 15.2.01, 552.3-Acri, CLSM 00594-XLIII. 43) LECCE, Cape Prosciutto, 0 m, on sheep dung, P. Franchi \& M. Marchetti, 23.11.01, 511.3-Torre Colimena, CLSM 00594-XLIV. 44) LECCE, Pisignano-Vernole, $40 \mathrm{~m}$, on sheep dung in d.c., V. Sciurti \& F. Doveri, 23.11.01, 513.4-S. Foca, CLSM 00594-XLV. 45) LECCE, Vanze, $10 \mathrm{~m}$, on cattle dung in d.c., V. Sciurti \& F. Doveri, 24.11.01, 513.4-S. Foca, CLSM 00594-XLVI. 46) LECCE, Vanze, 10 $\mathrm{m}$, on goat dung in d.c., V. Sciurti \& F. Doveri, 24.11.01, 513.4-S. Foca, CLSM 00594-XLVII. 47) LECCE, S. Foca, Le Cesine Wood, 0 m, on fox (?) dung in d.c., F. Doveri, 23.11.01, 513.4-S. Foca, CLSM 00594-XLVIII. 48) L'AQUILA, Campo di Giove, $1100 \mathrm{~m}$, on goat dung in d.c., E. Bizio, 20.9.01, 370.3-Cansano, CLSM 00594-XLIX. 49) VERONA, Bosco Chiesanuova-loc. S. Giorgio, $1500 \mathrm{~m}$, on cattle dung, A. Bizzi, 12.10.01, 102.3-Bosco Chiesanuova, CLSM 00594-L. 50) VICENZA, Gallio-loc. Malga Molina, 1600 m, on horse dung in d.c., A. Bizzi, 16.8.01, 082.1Monte Lisser, CLSM 00594-LI. 51) TREVISO, Borso-loc. Mount Grappa, 1200 m, on roe deer (?) dung in d.c., A. Bizzi, 10.8.01, 083.3-Monte Grappa, CLSM 00594-LII. 52) VICENZA, Gallio-loc. Malga Molina, $1600 \mathrm{~m}$, on sheep dung in d.c., A. Bizzi, 16.8.01, 082.1-Monte Lisser, CLSM 00594-LIII. 53) TREVISO, Borso-loc. Mount Grappa, Campo Croce, $1200 \mathrm{~m}$, on sheep dung in d.c., A. Bizzi, 10.8.01, 083.3-Monte Grappa, CLSM 00594-LIV. 54) COSENZA, Spezzano della Sila, Mount Scuro, 1600 m, on horse dung in d.c., C. Lavorato, 4.9.01, 560.3-Spezzano della Sila, CLSM 00594-LV. 55) COSENZA, Spezzano della Sila, Mount Scuro, 1600 m, on cattle dung in d.c., C. Lavorato, 4.9.01, 560.3-Spezzano della Sila, CLSM 00594-LVI. 56) COSENZA, Cozzo del Pesco, 1200 m, on cattle dung in d.c., C. Lavorato, 9.9.01, 561.4-Savelli, CLSM 00594-LVII. 57) COSENZA, Corigliano Calabro-loc. Baraccone, $1000 \mathrm{~m}$, on cattle dung in d.c., C. Lavorato, 4.11.01, 552.1-Corigliano Calabro, CLSM 00594-LVIII. 58) COSENZA, Fagnano Castello, 800 m, on roe deer dung in d.c., C. Lavorato, 23.10.01, 551.4-Fagnano Castello, CLSM 00594-LIX. 59) COSENZA, S. Giovanni in Fiore-loc. Carlo Magno, 1400 m, on sheep dung in d.c., C. Lavorato, 22.9.01, 560.1-Monte Volpintesta, CLSM 00594-LX. 60) COSENZA, Acri-Contrada Mischinella, $1100 \mathrm{~m}$, on wild pig dung in d.c., C. Lavorato, 19.10.01, 552.3-Acri, CLSM 00594-LXI. 61) MACERATA, Pintura di Bolognola, $1600 \mathrm{~m}$, on wild rabbit dung, F. Doveri, 3.5.02, 325.1Bolognola, CLSM 00594-LXII. 62) MACERATA, Pintura di Bolognola, $1600 \mathrm{~m}$, on horse dung, F. Doveri, 3.5.02, 325.1-Bolognola, CLSM 00594-LXIII. 63) TRENTO, Roncone, $850 \mathrm{~m}$, on goat dung, F. Doveri, 11.9.02, 080.4-Roncone, CLSM 00594-LXIV. 64) TRENTO, Roncone, $850 \mathrm{~m}$, on sheep dung in d.c., F. Doveri, 11.9.02, 080.4-Roncone, CLSM 00594-LXV. 65) BELLUNO, Lorenzago,, $900 \mathrm{~m}$, on roe deer dung, A. Bizzi, 22.7.02, 030.3-Pieve di Cadore, CLSM 00594LXVI. 66) BELLUNO, Falcade-Le Buse, ?, on bird dung in d.c., E. Bizio, 15.8.02, 045.1Cencenighe Agordino, CLSM 00594-LXVII. 67) COSENZA, Campana, $1000 \mathrm{~m}$, on horse dung in d.c., C. Lavorato, 8.6.02, 553.3-Bocchigliero, CLSM 00594-LXVIII. 68) COSENZA, Tarsia, 300 $\mathrm{m}$, on sheep dung in d.c., C. Lavorato, 10.5.02, 543.2-Spezzano Albanese, CLSM 00594-LXIX. 69) COSENZA, Acri, $1000 \mathrm{~m}$, on wild pig dung in d.c., C. Lavorato, 10.5.02, 552.3-Acri, CLSM 00594-LXX. 70) PISA, S. Ruffino, 50 m, on sheep dung in d.c., F. Doveri, 22.3.03, 284.1-Casciana Terme, CLSM 00594-LXXI. 71) PISA, Chianni, 300 m, on sheep dung in d.c., F. Doveri, 22.3.03, 
284.2-Chianni, CLSM 00594-LXXII. 72) LECCO, Concenedo, $920 \mathrm{~m}$, on cattle dung in d.c., F. Doveri, 6.6.03, 076.4-Barzio, CLSM 00594-LXXIII. 73) LECCO, Premana (Piano di Camaggiore), $1190 \mathrm{~m}$, on cattle dung in d.c., A. Bizzi, 7.6.03, 055.3-Premana, CLSM 00594-LXXIV. 74) REGGIO CALABRIA, Tazza, $800 \mathrm{~m}$, on cattle dung, F. Doveri, 17.10.03, 602.3-Motta San Giovanni, CLSM 00594-LXXV. 75) REGGIO CALABRIA, Passo Petrulli (Aspromonte), 1100 m, on sheep dung, F. Doveri, 17.10.03, 602.4-Santo Stefano in Aspromonte, CLSM 00594-LXXVI. 76) UDINE, Piani Montasio, $1800 \mathrm{~m}$, on marmot dung in d.c., L. Levorato, 2.7.03, 033.3-Saletto, CLSM 00594-LXXVII. 77) BELLUNO, Croce d'Aune pass, $2000 \mathrm{~m}$, on roe deer dung, A. Bizzi, 22.6.03, 062.3-Fonzaso, CLSM 00594-LXXVIII. 78) PORDENONE, Pinedo (Val Settimana), 1200 $\mathrm{m}$, on cattle dung in d.c., A. Bizzi, 27.7.03, 047.2-Claut, CLSM 00594-LXXIX. 79) PORDENONE, Pinedo (Val Settimana), $1200 \mathrm{~m}$, on deer dung in d.c., A. Bizzi, 27.7.03, 047.2-Claut, CLSM 00594-LXXX. 80) BELLUNO, Croce d'Aune pass, $2000 \mathrm{~m}$, on sheep dung in d.c., A. Bizzi, 22.6.03, 062.3-Fonzaso, CLSM 00594-LXXXI. 81) VERONA, Molina, $800 \mathrm{~m}$, on horse dung, A. Bizzi, 18.9.03, 101.2-Dolcè, CLSM 00594-LXXXII. 82) VICENZA, Recoaro, $1000 \mathrm{~m}$, on rabbit dung in d.c., A. Bizzi, 22.5.04, 102.2-Valdagno, CLSM 00594-LXXXIII. 83) VICENZA, Recoaro, $1000 \mathrm{~m}$, on horse dung in d.c., A. Bizzi, 22.5.04, 102.2-Valdagno, CLSM 00594-LXXXIX. 84) CAMPOBASSO, Campitello di Sepino, 1350 m, on horse dung in d.c., F. Doveri, 14.10.04, 405.2Vinchiaturo, CLSM 00594-XC. 85) CAMPOBASSO, Campitello di Sepino, $1350 \mathrm{~m}$, on cattle dung in d.c., F. Doveri, 14.10.04, 405.2-Vinchiaturo, CLSM 00594-XCI. 86) CAMPOBASSO, Vinchiaturo, $500 \mathrm{~m}$, on sheep dung in d.c., F. Doveri, 14.10.04, 405.2-Vinchiaturo, CLSM 00594XCII. 87) VICENZA, Arsiero, Malga Melegnon, 1300 m, on cattle dung, A. Bizzi, 3.9.04, 081.1Caldonazzo, CLSM 00594-XCIII. 88) VICENZA, Malga Melegnon, $600 \mathrm{~m}$, on roe deer dung in d.c., A. Bizzi, 3.9.04, 081.1-Caldonazzo, CLSM 00594-XCIV. 89) COSENZA, Celico, loc. Lagarò, $1200 \mathrm{~m}$, on horse dung in d.c., C. Lavorato, 21.3.05, 560.4-Celico, CLSM 00594-XCV. 90) COSENZA, S. Fili-Carrera, ? m, on sheep dung in d.c., C. Lavorato, 16.11.04, 559.1-Rende, CLSM 00594-XCVI. 91) COSENZA, Longobucco, $1000 \mathrm{~m}$, on sheep dung in d.c., C. Lavorato, 10.8.04, 552.2-Longobucco, CLSM 00594-XCVII. 92) VICENZA, Sarego, Mount Roccolo, $250 \mathrm{~m}$, on sheep dung, A. Bizzi, 22.4.05, 125.2-Longare, CLSM 00594-XCVIII. 93) VICENZA, Sarego, Grandona-Mount Cocco, 230 m, on sheep dung, A. Bizzi, 13.4.05, 125.2-Longare, CLSM 00594XCIX. 94) CUNEO, Viola, loc. il Colletto, $1100 \mathrm{~m}$, on cattle dung, A. Bizzi, 14.9.05, 227.2Pamperato, CLSM 00594-C. 95) VICENZA, Grancona, Mount Caldiero, ? $\mathrm{m}$, on sheep dung in d.c., A. Bizzi, 7.8.05, 125.3-Montebello Vicentino, CLSM 00594-CI. 96) VICENZA, Grancona, Mount Caldiero, ? m, on sheep dung in d.c., A. Bizzi, 7.8.05, 125.3-Montebello Vicentino, CLSM 00594-CII. 97) LIVORNO, Lorenzago, 1600 m, on sheep dung, A. Bizzi, 26.6.05, 030.3-Pieve di Cadore, CLSM 00594-CIII. 98) BELLUNO Lorenzago, Casera Razzo, $1800 \mathrm{~m}$, on goat dung in d.c., A. Bizzi, 26.6.05, 030.3-Pieve di Cadore, CLSM 00594-CIV. 99) LIVORNO, Bibbona, La Pira farm holidays, $50 \mathrm{~m}$, on sheep dung, F. Doveri, 21.5.06, 294.2-Bibbona, CLSM 00594-CVI. 100) L'AQUILA, Piani del Sirente, $1050 \mathrm{~m}$, on hare dung in d.c., F. Doveri, 9.6.06, 368.1Ovindoli, CLSM 00594-CVII. 101) L'AQUILA, Celano, $800 \mathrm{~m}$, on roe deer dung in d.c., F. Doveri, 9.6.06, 368.2-Celano, CLSM 00594-CVIII. 102) FERRARA, Mesola wood, $0 \mathrm{~m}$, on fallow deer dung in d.c., A. Bizzi, 12.1.05, 187.1-Mesola, CLSM 00594-CIX. 103) VICENZA, Valdagno, Zovo pass, $600 \mathrm{~m}$, on horse dung in d.c., A. Bizzi, 18.5.06, 102.2-Valdagno, CLSM 00594-CX. 104) VERONA, Podesteria, $1600 \mathrm{~m}$, on cattle dung in d.c., A. Bizzi, 1.6.06, 102.3-Bosco Chiesanuova, CLSM 00594-CXI. 105) TRENTO, Mount Baldo, malga Gambon, $1250 \mathrm{~m}$, on horse dung in d.c., A. Bizzi, 25.5.06, 080.2-Riva del Garda, CLSM 00594-CXII. 106) TRENTO, Mount Baldo, $1400 \mathrm{~m}$, on cattle dung in d.c., A. Bizzi, 25.5.06, 080.2-Riva del Garda, CLSM 00594CXIII. 107) TRENTO, Brentonico, $1500 \mathrm{~m}$, on cattle dung in d.c., A. Bizzi, 25.5.06, 080.2-Riva del Garda, CLSM 00594-CXIV. 108) VERONA, Branchetto pass, $1600 \mathrm{~m}$, on cattle dung in d.c., A. Bizzi, 1.6.06, 102.3-Bosco Chiesanova, CLSM 00594-CXV. 109) TRENTO, Folgaria, Forte Kerle, $1300 \mathrm{~m}$, on cattle dung, A. Bizzi, 12.10.06, 081.3-Rovereto, CLSM 00594-CXVI. 110) VICENZA, Recoaro Terme, Mount Rasta, 950 m, on sheep dung in d.c., F. Doveri, 8.6.07, 102.1-Recoaro 
Terme, CLSM 00594-CXVII. 111) VICENZA, Recoaro Terme, Mount Rasta, $950 \mathrm{~m}$, on horse dung in d.c., F. Doveri, 8.6.07, 102.1-Recoaro Terme, CLSM 00594-CXVIII. 112) VICENZA, Recoaro Terme, Mount Rasta, $950 \mathrm{~m}$, on sheep dung in d.c., F. Doveri, 8.6.07, 102.1-Recoaro Terme, CLSM 00594-CXIX. 113) VICENZA, Recoaro Terme, Mount Rasta, 950 m, on horse dung in d.c., F. Doveri, 8.6.07, 102.1-Recoaro Terme, CLSM 00594-CXX. 114) L'Aquila, Gran Sasso, Campo Imperatore, $2400 \mathrm{~m}$, on roe deer dung in d.c., L. Levorato, 11.5.07, 349.2-Gran Sasso d'Italia, CLSM 00594-CXXI. 115) TRENTO, Folgarida, Malga Folgarida, 1600 m, on cattle dung, F. Doveri, 6.9.07, 042.2-Lago di Tovel, CLSM 00594-CXXII. 116) TRENTO, Dimaro, Malga di Sadron, 1450 m, on cattle dung in d.c., F. Doveri, 8.9.07, 042.1-Malé, CLSM 00594-CXXIII. 117) TRENTO, Folgarida, Malga Folgarida, 1600 m, on goat dung in d.c., F. Doveri, 6.9.07, 042.2-Lago di Tovel, CLSM 00594-CXXIV. 118) TRENTO, Folgarida, Malga Folgarida, $1600 \mathrm{~m}$, on donkey dung in d.c., F. Doveri, 6.9.07, 042.2-Lago di Tovel, CLSM 00594-CXXV. 119) TRENTO, Dimaro, Malghetta, $1100 \mathrm{~m}$, on hare dung in d.c., F. Doveri, 8.9.07, 042.1-Malé, CLSM 00594CXXVI. 120) VICENZA, Faldo, Mount Malo, $200 \mathrm{~m}$, on horse dung in d.c., A. Bizzi, 1.9.07, 102.2-Valdagno, CLSM 00594-CXXVII. 121) REGGIO EMILIA, Pratizzano pass, $1200 \mathrm{~m}$, on hare dung in d.c., L. Levorato, 23.5.08, 217.2-Ramiseto, 00594-CXXVIII. 122) TRENTO, Tres, Mount Corno, $1600 \mathrm{~m}$, on roe deer dung in d.c., A. Bizzi, 4.10.08, 043.4-Cles, CLSM 00594-CXXIX. 123) VICENZA, Lastebasse, Fiorentini, $1500 \mathrm{~m}$, on cattle dung in d.c., A. Bizzi, 13.7.08, 081.1Caldonazzo, CLSM 00594-CXXX. 124) LIVORNO, Bibbona, Faltona farm, 50 m, on sheep dung in d.c., 18.3.09, 295.3-Sassa, CLSM 00594-CXXXI. 125) PISA, Orciatico, $50 \mathrm{~m}$, on sheep dung in d.c., F. Doveri, 13.4.09, 285.3-Lajatico, CLSM 00594-CXXXII. 126) VICENZA, Solagna, Campeggia, $1000 \mathrm{~m}$, on mouflon dung in d.c., A. Bizzi, 2.6.09, 083.3-Monte Grappa, CLSM 00594-CXXXIII. 127) NOVARA, Druogno, Santa Maria, $850 \mathrm{~m}$, on horse dung, F. Doveri, 15.5.10, 116.1-Bellinzago, CLSM 00594-CXXXIV. 128) NOVARA, Alpe Devero, Baceno, 1600 $\mathrm{m}$, on chamois dung in d.c., D. Bolognini \& G. Baiano, 15.5.10, 035.1-Alpe Devero, CLSM 00594CXXXV. 129) NOVARA, Piana di Vigezzo, $1700 \mathrm{~m}$, on horse dung in d.c., F. Ferigo, 15.5.10, 052.4-Santa Maria Maggiore, CLSM 00594-CXXXVI. 130)VERONA, Ferrara di Monte Baldo, $1300 \mathrm{~m}$, on hare dung in d.c., L. Levorato, 11.8.10, 101.3-Caprino Veronese, CLSM 00594CXXXVII. 131) GROSSETO, Collacchia, 50 m, on sheep dung in d.c., F. Doveri, 25.3.11, 319.4Ribolla, CLSM 00594-CXXXVIII. 132) GROSSETO, Monterotondo Marittimo, Capannini, 100 m, on sheep dung in d.c., F. Doveri, 25.3.11, 306.4-Monterotondo Marittimo, CLSM 00594-CXXXIX. 133) GROSSETO, Monterotondo Marittimo, boraciferous lake, $250 \mathrm{~m}$, on horse dung in d.c., F. Doveri, 25.3.11, 306.4-Monterotondo Marittimo, CLSM 00594-CXL. 134) GROSSETO, Monterotondo Marittimo, boraciferous lake, $250 \mathrm{~m}$, on cattle dung in d.c., F. Doveri, 25.3.11, 306.4-Monterotondo Marittimo, CLSM 00594-CXLI. 135) GROSSETO, Gavorrano rail-way station, $70 \mathrm{~m}$, on horse dung in d.c., F. Doveri, 25.3.11, 318.1-Gavorrano, CLSM 00594-CXLII. 136) GROSSETO, Monterotondo Marittimo, boraciferous lake, $250 \mathrm{~m}$, on cattle dung in d.c., F. Doveri, 25.3.11, 306.4-Monterotondo Marittimo, CLSM 00594-CXLIII. 137) LIVORNO, Elba island, Campo nell'Elba, Prataccio, Valle Buia, $200 \mathrm{~m}$, on hare dung in d.c., C. Cotta, 15.9.11, 328.1-Marina di Campo, CLSM 00594-CXLIV. 138) TRENTO, Vervò, Non valley, Predaia refuge, $1300 \mathrm{~m}$, on hare dung in d.c., A. Bizzi, 3.10.10, 043.4-Cles, CLSM 00594-CXLV. 139) TRENTO, Vervò, Non valley, Predaia refuge, 1300 m, on roe deer dung in d.c., A. Bizzi, 3.10.10, 043.4-Cles, CLSM 00594-CXLVI.

TOTAL 139: sheep 39; cattle 25; horse 23; roe deer 11 ; goat 7 ; hare 7 ; rock goat 5 ; donkey 3 ; marmot 3; rabbit 3; wild pig 3; deer 2; fallow deer 2; fox 2; bird 1; chamois 1 ; mouflon 1 ; wolf 1 .

Ascobolus lineolatus Brumm., Persoonia, suppl. 1: 120, 1967.

Figs 79-81

Material examined - 1) LECCE, Punta Prosciutto, $0 \mathrm{~m}$, seven scattered, superficial specimens on fox (?) dung in d.c., P. Franchi \& M. Marchetti, 25.11.01, 511.3-Torre Colimena, CLSM 007.02. 2) PISA, La Sassa, 350 m, on roe deer dung in d.c., F. Doveri, 15.10.12, 295.3Sassa, CLSM 007.02 ter. 3) LIVORNO, Quercianella, 0 m, on fallow deer dung in d.c., F. Doveri, 


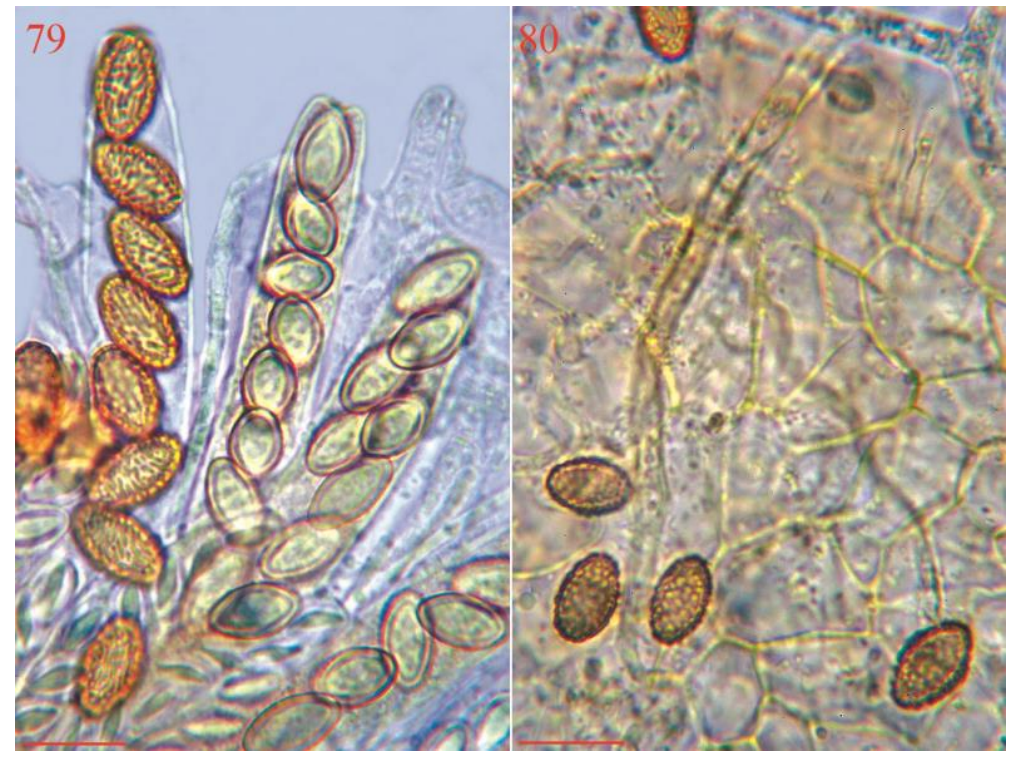

Figs 79-80 - Ascobolus lineolatus. 79 Asci with ascospores in different stages of maturity. 80 Detail of ectal excipulum and some free ascospores. - Bars $73-74=50 \mu \mathrm{m} .75,77-78=25 \mu \mathrm{m} .76$ $=60 \mu \mathrm{m}$.

19.12.12, 284.3-Rosignano Marittimo, CLSM 007.02 quater. 4) LIVORNO, Quercianella, 0 m, on roe deer dung in d.c., F. Doveri, 19.12.12, 284.3-Rosignano Marittimo, CLSM 007.02 penta. 5) LIVORNO, Quercianella, $0 \mathrm{~m}$, on wild pig dung in d.c., F. Doveri, 31.5.13, 284.3-Rosignano Marittimo, CLSM 007.02 esa. 6) LIVORNO, Quercianella, $50 \mathrm{~m}$, on wild pig dung in d.c., F. Doveri, 31.5.13, 284.3-Rosignano Marittimo, CLSM 007.02 epta.

TOTAL 6: roe deer 2; wild pig 2; fallow deer 1; fox 1.

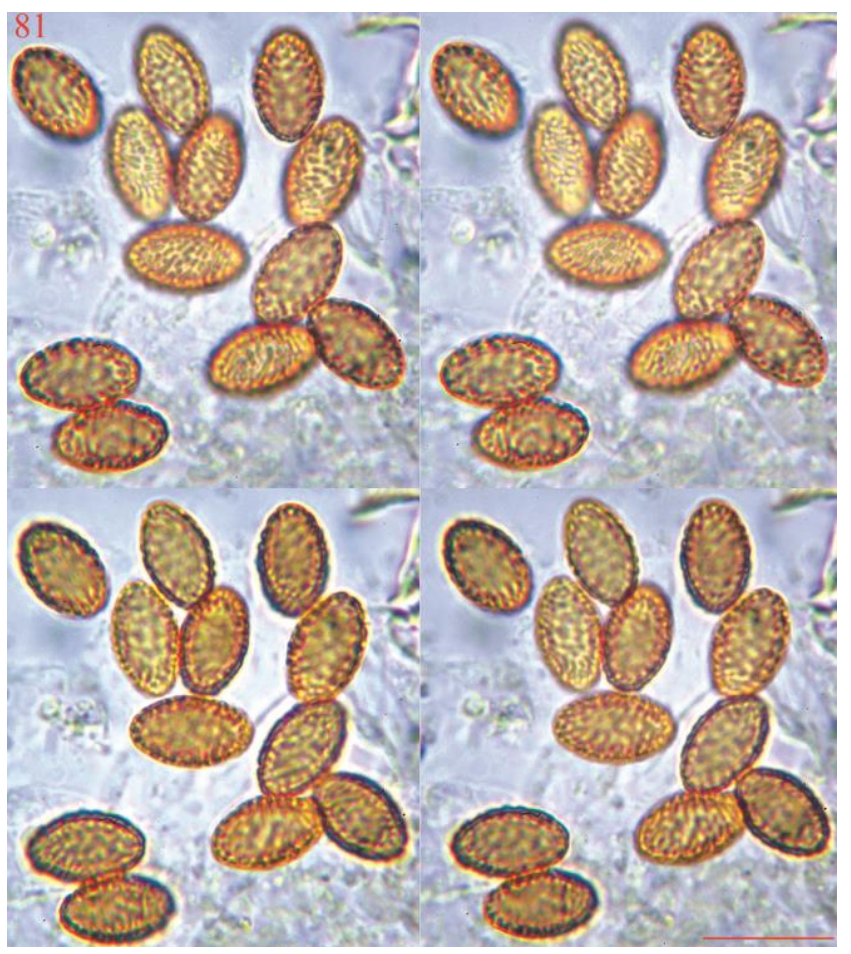

Fig 81 - Ascobolus lineolatus. Ascospores in four different focuses. - Bar $=12 \mu \mathrm{m}$. 


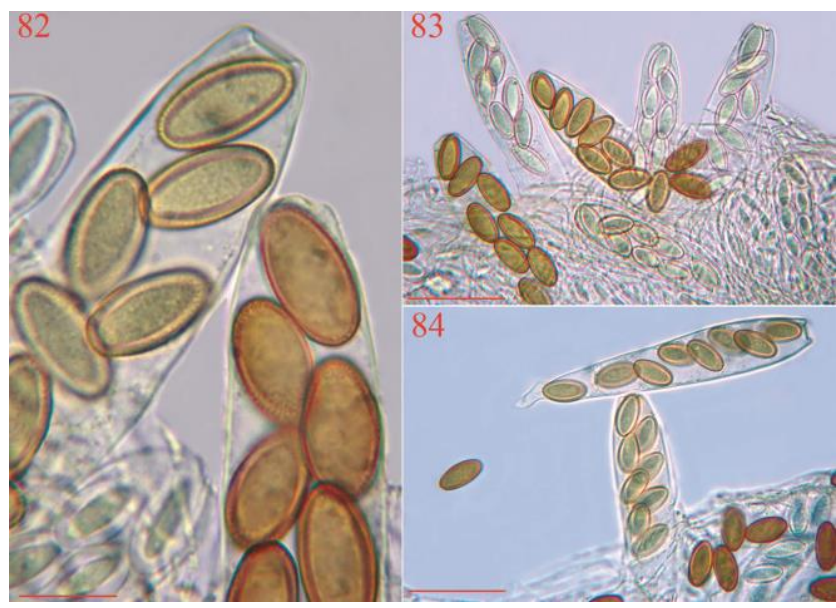

Figs 82-84 - Ascobolus mancus. Asci with ascospores in different stages of maturity. - Bars $82=$ $15 \mu \mathrm{m} .83-84=50 \mu \mathrm{m}$.

Material examined - 1) PISA, Calambrone stables, $0 \mathrm{~m}$, about ten gregarious, superficial specimens, on horse dung in d.c., F. Doveri, 30.11.95, 272.2-Marina di Pisa, MCVE 495. 2) COSENZA, Orsomarso, $1300 \mathrm{~m}$, on donkey dung in d.c., C. Lavorato, 17.5.98, 533.2-Mormanno, CLSM 00896 bis. 3) COSENZA, Corigliano, $300 \mathrm{~m}$, on goat dung in d.c., C. Lavorato, 16.5.98, 552.1-Corigliano Calabro, CLSM 00896 ter. 4) LECCE, Vanze, $10 \mathrm{~m}$, on cattle dung in d.c., V. Sciurti \& F. Doveri, 24.11.01, 513.4-S. Foca, CLSM 00896 quater. 5) VICENZA, Barberino, S. Giovanni in Monte, ? m, on horse dung in d.c., A. Bizzi, 26.11.05, 125.2-Longare, CLSM 00896 penta. 6) PISA, San Miniato Basso, 0 m, on horse dung in d.c., F. Doveri, 13.4.09, 274.2-San Miniato, CLSM 00896 esa.

TOTAL 6: horse 3; cattle 1; donkey 1; goat 1.

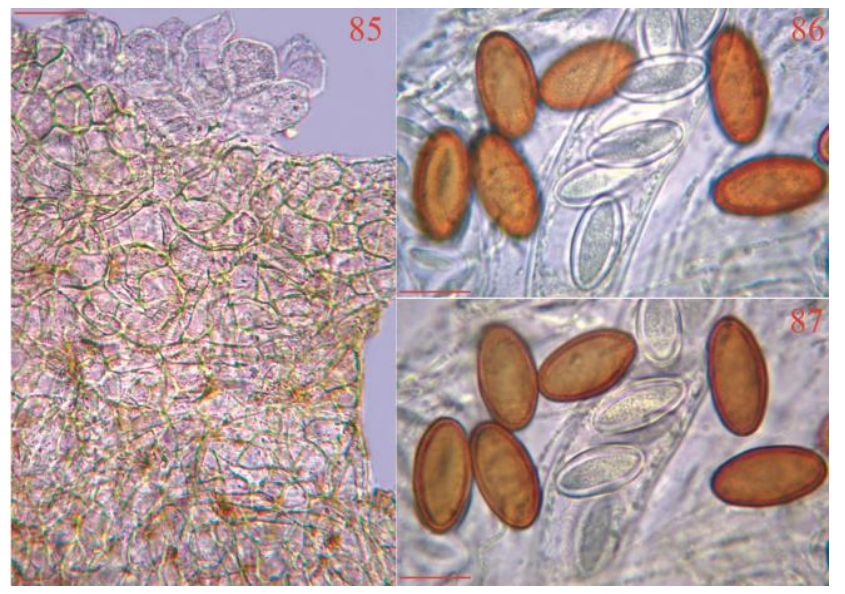

Figs 85-87 - Ascobolus mancus. 85 Detail of medullary (above) and ectal (below) excipulum. 8687 Ascospores in different focuses. - Bars $85=15 \mu \mathrm{m} .86-87=12 \mu \mathrm{m}$.

Ascobolus michaudii Boud., Hist. Class. Discom. Eur.: 71- 72, 1907.

Figs 88-91

Material examined - 1) COSENZA, Acri (loc. S. Zagaria), $800 \mathrm{~m}$, about thirty scattered or gregarious, superficial specimens, on donkey dung in d.c., C. Lavorato, 3.9.01, 552.3-Acri, CLSM 015.01. 2) REGGIO CALABRIA, Tazza, 800 m, on cattle dung in d.c., F. Doveri, 17.10.03, 602.3Motta San Giovanni, CLSM 015.01 bis. 3) REGGIO CALABRIA, Cardito (Aspromonte), $900 \mathrm{~m}$, on ostrich dung in d.c., A. Bizzi, 16.10.03, 602.1-Gambarie, CLSM 015.01 ter. 4) PORDENONE, 


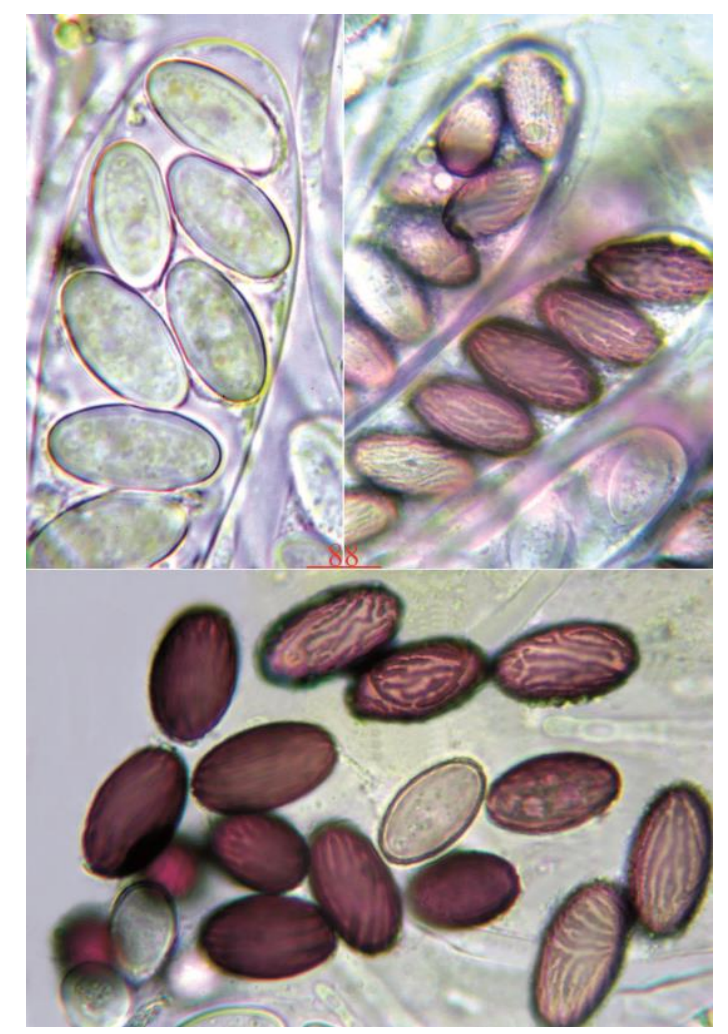

Fig 88 - Ascobolus michaudii. Free ascospores and ascospores inside asci in different stages of maturity. - Bar $10 \mu \mathrm{m}$.

Pinedo (Val Settimana), $1200 \mathrm{~m}$, on deer dung in d.c., A. Bizzi, 27.7.03, 047.2-Claut, CLSM 015.01 quater. 5) VICENZA, Recoaro, $1000 \mathrm{~m}$, on rabbit dung in d.c., A. Bizzi, 22.5.04, 102.2Valdagno, CLSM 015.01-esa. 6) CAMPOBASSO, Vinchiaturo, $500 \mathrm{~m}$, on sheep dung in d.c., F. Doveri, 14.10.04, 405.2-Vinchiaturo, CLSM 015.01 epta. 7) COSENZA, Longobucco, $1000 \mathrm{~m}$, on sheep dung in d.c., C. Lavorato, 10.8.04, 552.2-Longobucco, CLSM 015.01-octo. 8) VICENZA, Barberino, S. Giovanni in Monte, ? m, on horse dung in d.c., A. Bizzi, 26.11.05, 125.2-Longare, CLSM 015.01-ena. 9) UDINE, Ampezzo, Nount Sesilis, 1500 m, on sheep dung in d.c., A. Bizzi, 25.6.05, 031.3-Ampezzo, CLSM 015.01 deca. 10) LIVORNO, Bibbona, La Pira farm holidays, 50 $\mathrm{m}$, on sheep dung, F. Doveri, 21.5.06, 294.2-Bibbona, CLSM 015.01-XI. 11) L'AQUILA, Celano, $800 \mathrm{~m}$, on sheep dung in d.c., F. Doveri, 9.6.06, 368.2-Celano, CLSM 015.01-XII. 12) VENEZIA, Chioggia, Nordio wood, $0 \mathrm{~m}$, on fallow deer dung in d.c., L. Levorato, 8.4.06, 148.2-Chioggia, CLSM 015.01-XIII. 13) VERONA, Soave, 350 m, on horse dung in d.c., A. Bizzi, 17.4.06, 124.2Soave, CLSM 015.01-XIV. 14) VICENZA, Faldo, Mount Malo, 500 m, on horse dung in d.c., A. Bizzi, 2.12.06, 102.2-Valdagno, CLSM 015.01-XV. 15) TRENTO, Folgarida, Malga Folgarida, $1600 \mathrm{~m}$, on cattle dung in d.c., F. Doveri, 6.9.07, 042.2-Lago di Tovel, CLSM 015.01-XVI. 16) VENEZIA, Caorle, loc. Brussa, $0 \mathrm{~m}$, on horse dung in d.c., A. Bizzi, 27.10.07, 129.1-Caorle, CLSM 015.01-XVII. 17) LIVORNO, Suvereto, La Suveraia farm, $0 \mathrm{~m}$, on horse dung in d.c., F. Doveri, 18.3.09, 306.3-Suvereto, CLSM 015.01-XVIII. 18) PISA, Volterra, Villamagna, Vallicella farm, $200 \mathrm{~m}$, on sheep dung in d.c., F. Doveri, 13.4.09, 285.3-Lajatico, CLSM 015.01-XIX. 19) VICENZA, Solagna, Campeggia, $1000 \mathrm{~m}$, on mouflon dung in d.c., A. Bizzi, 2.6.09, 083.3-Monte Grappa, CLSM 015.01-XX. 20) NOVARA, Druogno, Santa Maria, 850 m, on horse dung in d.c., F. Doveri, 15.5.10, 116.1-Bellinzago, CLSM 015.01-XXI. 21) VENEZIA, Caroman, $0 \mathrm{~m}$, on peewit gull dung (Larus sp.) in d.c., L. Levorato, 18.4.10, 148.2-Chioggia, CLSM 015.01-XXII. 22) GROSSETO, Monterotondo Marittimo, boraciferous lake, $250 \mathrm{~m}$, on cattle dung in d.c., F. Doveri, 25.3.11, 306.4-Monterotondo Marittimo, CLSM 015.01-XXIII. 23) GROSSETO, Gavorrano railway station, $70 \mathrm{~m}$, on cattle dung in d.c., F. Doveri, 25.3.11, 318.1-Gavorrano, CLSM 015.01- 
XXIV. 24) GROSSETO, Monterotondo Marittimo, boraciferous lake, $250 \mathrm{~m}$, on cattle dung in d.c., F. Doveri, 25.3.11, 306.4-Monterotondo Marittimo, CLSM 015.01-XXV. 25) VENEZIA, Chioggia, Nordio wood, 0 m, on roe deer dung in d.c., Cerello R. and Robich G., 27.4.13, 148.2-Chioggia, CLSM 015.01-XXVI.

TOTAL 25: horse 6; sheep 6; cattle 5; bird 1; deer 1; donkey 1 ; fallow deer 1 ; mouflon 1 ; ostrich 1 ; rabbit 1 ; roe deer 1 .

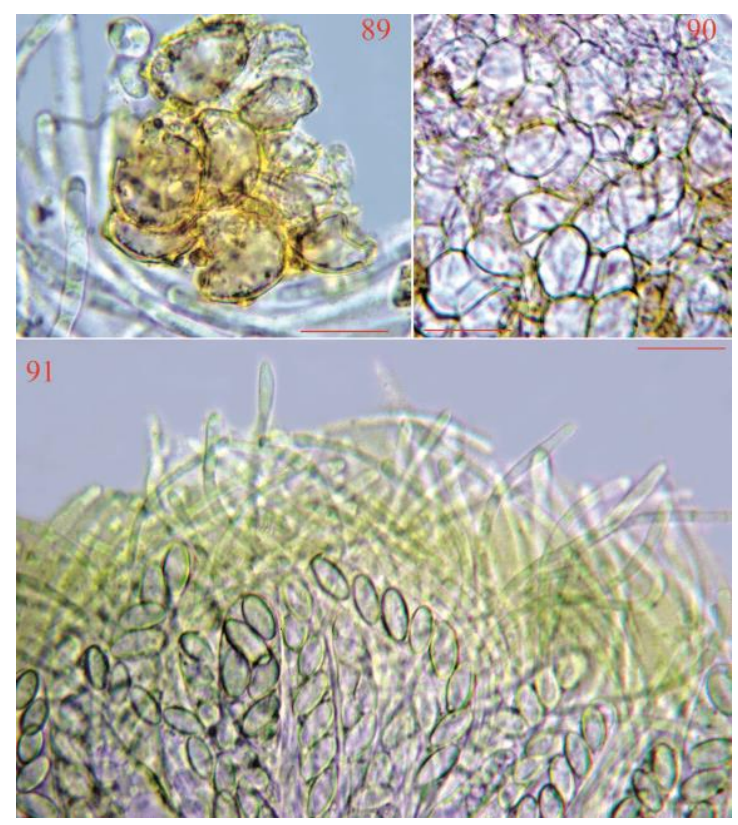

Figs 89-91 - Ascobolus michaudii. 89 Globular cells of an excipular grain. 90 Detail of ectal excipulum. 91 Hymenial surface with paraphyses embedded in a yellow gelatinous material. - Bars $89=15 \mu \mathrm{m} .90=50 \mu \mathrm{m} .91=30 \mu \mathrm{m}$.

Ascobolus perforatus Brumm., Persoonia 11: 356, 1981.

Figs 92-93

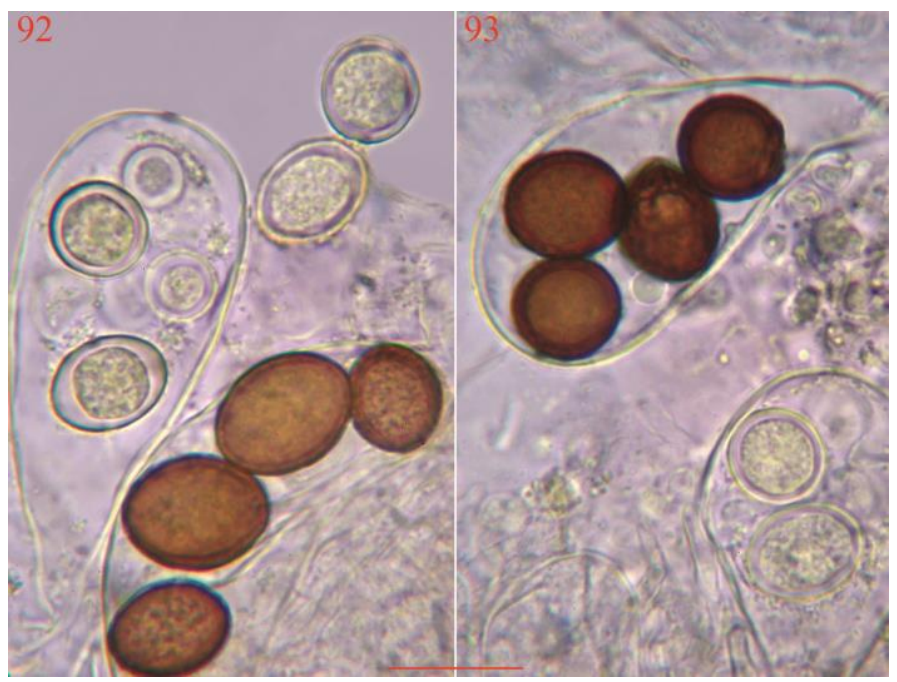

Figs 92-93 - Ascobolus perforatus. 92-93 Asci with ascospores in different stages of maturity. $\mathrm{Bar}=20 \mu \mathrm{m}$.

Material examined - PISA, Monteverdi Marittimo, Allumiere farm, $200 \mathrm{~m}$, six scattered, superficial specimens, on rabbit dung in d.c., F. Doveri, 25.3.11, 306.4-Monteverdi Marittimo, CLSM 006.11. 
Ascobolus aff. pseudocainii Prokhorov, Mikol. Fitopat. 24: 404, 1990.

Figs 94-98

Material examined - 1) UDINE, Fleons gorge, $1400 \mathrm{~m}$, about ten gregarious, superficial specimens, on deer dung in d.c, F. Bersan \& F. Doveri, 26.6.98, 031.1-Rigolato, CLSM 04998. 2) BELLUNO, Croce d'Aune pass, 2000 m, on roe deer dung in d.c., A. Bizzi, 22.6.03, 062.3-Fonzaso, CLSM 04998 bis.

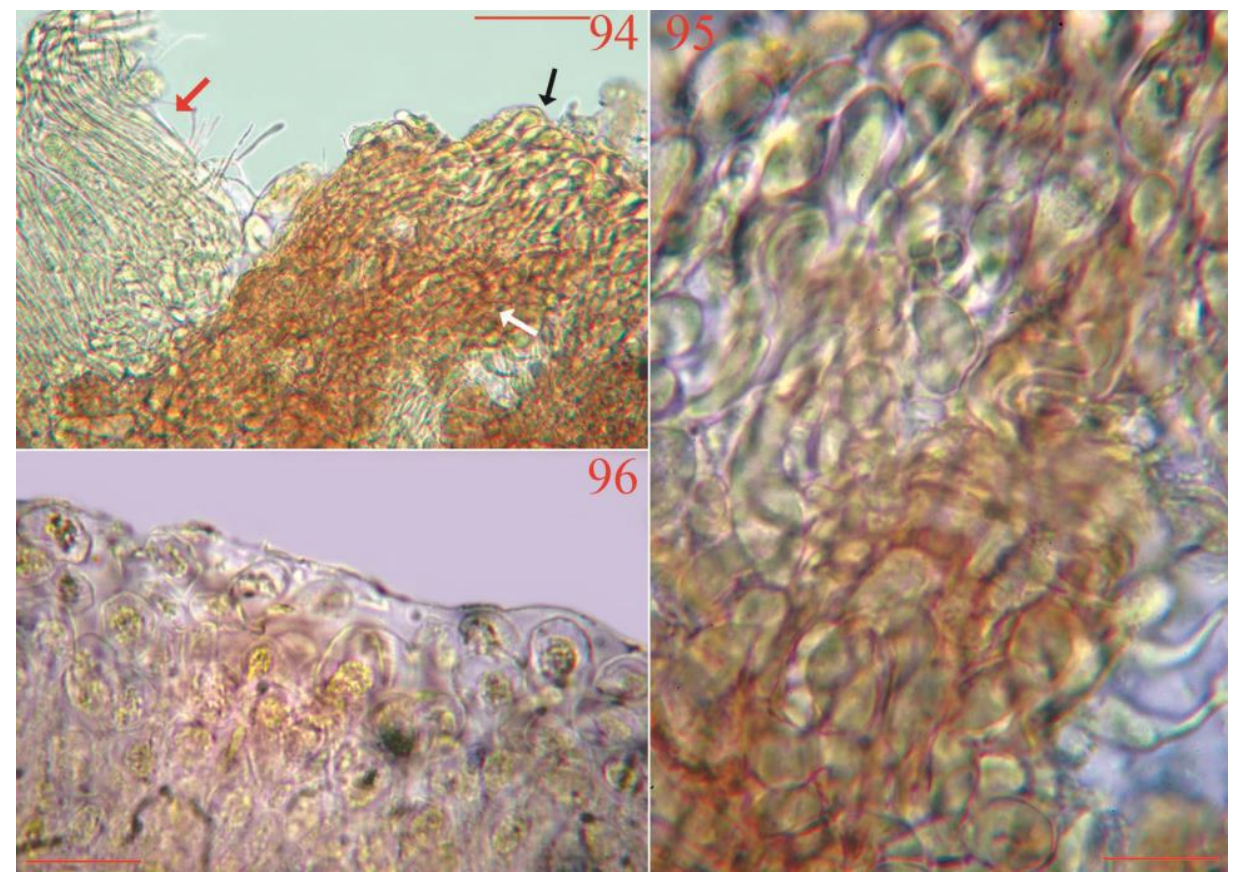

Figs 94-96 - Ascobolus aff. pseudocainii. 94 Ectal excipulum in the upper part (white arrow) and at the margin (black arrow) of receptacle. 95 Detail of ectal excipulum at the margin. 96 Paraphyses embedded in a hyaline gelatinous material. - Bars $94=60 \mu \mathrm{m} .95=30 \mu \mathrm{m} .96=12 \mu \mathrm{m}$.

TOTAL 2: deer 1; roe deer 1.

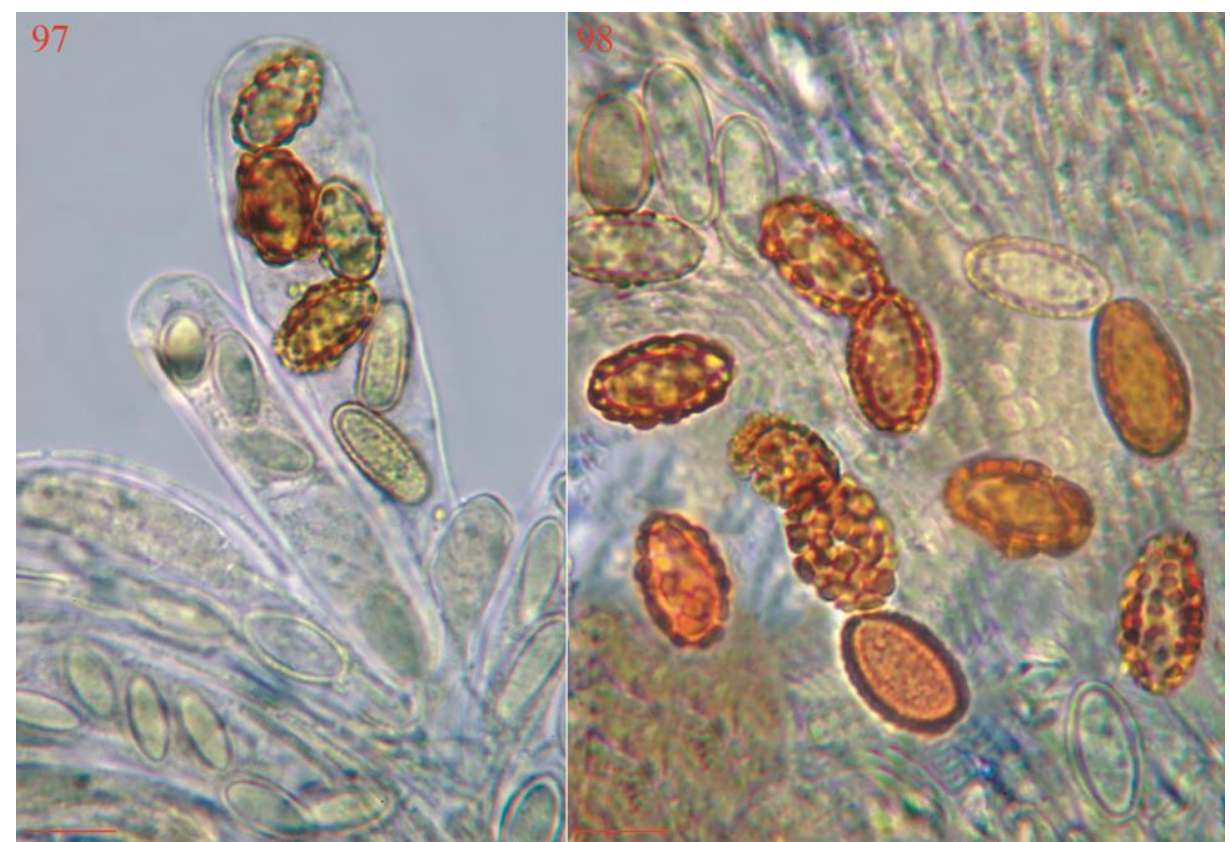

Figs 97-98 - Ascobolus aff. pseudocainii. 97 Asci with ascospores in different stages of maturity. 98 Free ascospores. - Bars $97=10 \mu \mathrm{m} .98=8 \mu \mathrm{m}$. 


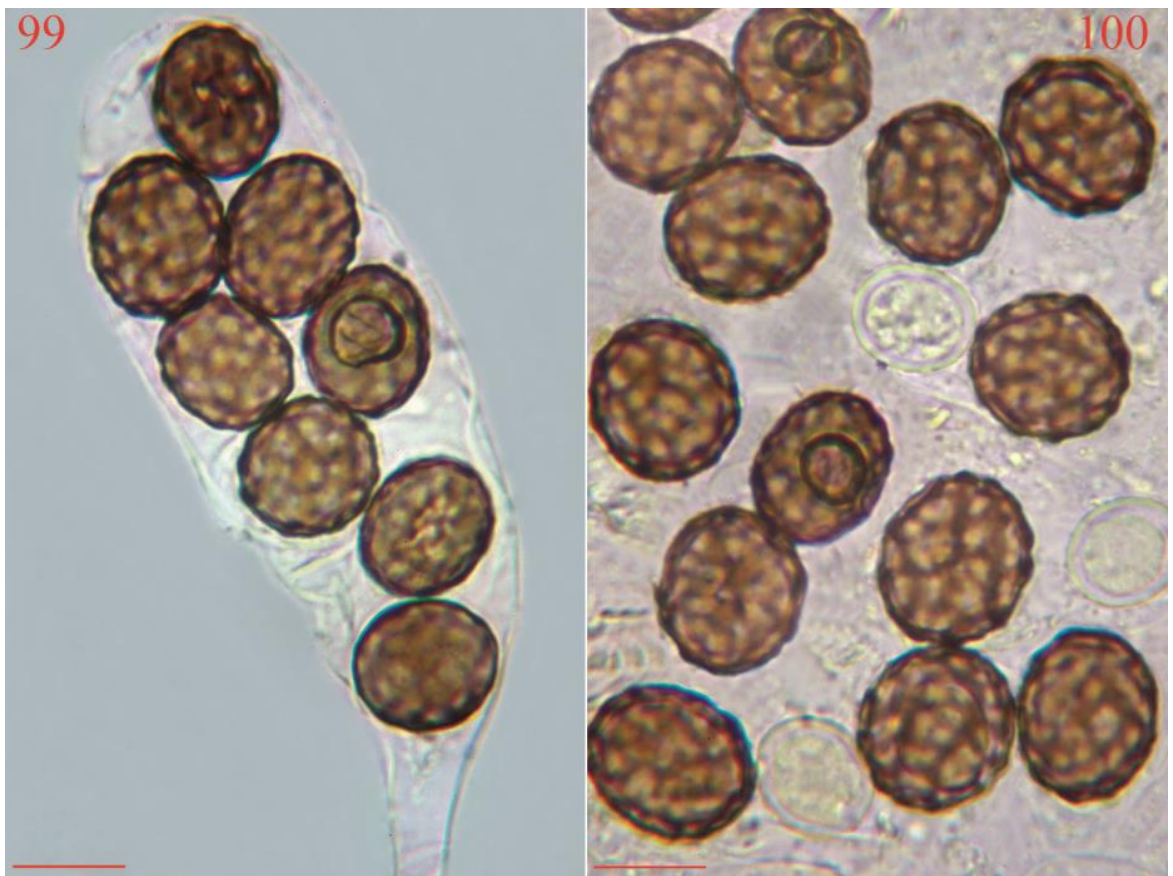

Figs 99-100 - Ascobolus reticulatus. 99 Ascus with biseriate ascospores. 100 Mature ascospores. Bars $99=12 \mu \mathrm{m} .100=10 \mu \mathrm{m}$.

Material examined - LIVORNO, Bibbona, La Pira farm holidays, $50 \mathrm{~m}$, about ten solitary, superficial specimens, on goose dung in culture, F. Doveri, 21.5.06, 294.2-Bibbona, CLSM 004.06.

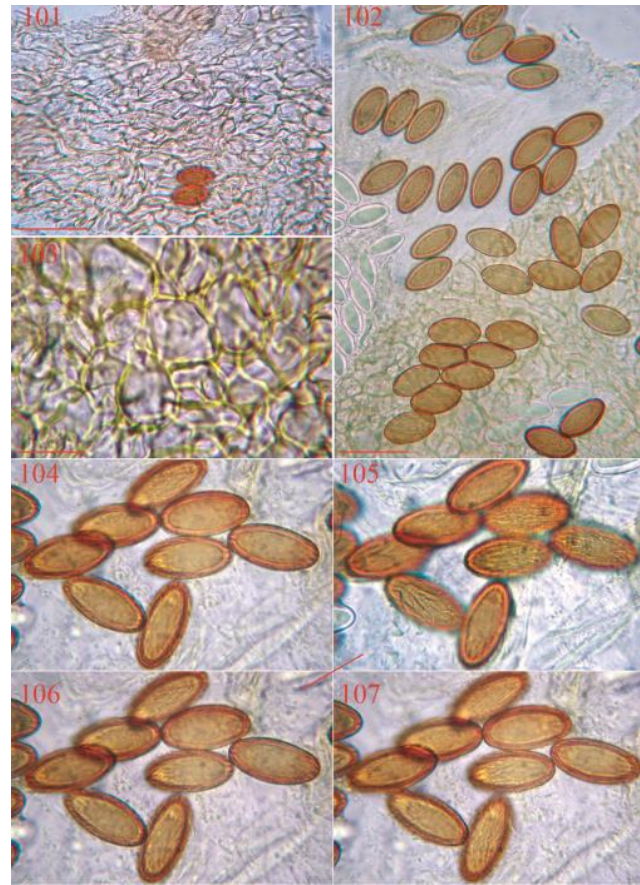

Figs 101-107 - Ascobolus roseopurpurascens. 101 Upper part of ectal excipulum. 102 Mature ascospores in the foreground. 103 Detail of the lower part of ectal excipulum. 103-107 Mature ascospores in different focuses. Bars $101=50 \mu \mathrm{m} .102=35 \mu \mathrm{m} .103,104-107=20 \mu \mathrm{m}$. 
Material examined - TRENTO, Roncone, dozens of gregarious, often crowded, superficial specimens on dung of an unidentified herbivore, G. Medardi \& C. Gallinari, 23.1.99, 079.1-Pieve di Bono, CLSM 00499.

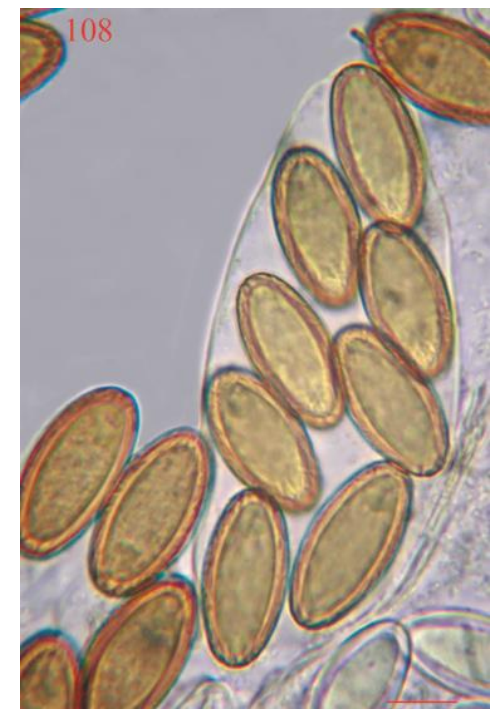

Fig 108 - Ascobolus roseopurpurascens. Ascus with biseriate ascospores, and free ascospores. Bar $=10 \mu \mathrm{m}$.

Ascobolus sacchariferus

Figs 11-19

Material examined - 1) VENEZIA, Caroman, $0 \mathrm{~m}$, about fifty supericial, gregarious specimens on peewit gull dung (Larus sp.) in d.c., L. Levorato, 18.4.10, 148.2-Chioggia, CLSM 004.11. 2) GROSSETO, Monterotondo Marittimo, boraciferous lake, $250 \mathrm{~m}$, on cattle dung in d.c., F. Doveri, 25.3.11, 306.4-Monterotondo Marittimo, CLSM 004.11 bis. 3) PISA, La Sassa, 350 m, on roe deer dung in d.c., F. Doveri, 15.10.12, 295.3-Sassa, CLSM 004.11 ter. 4) LIVORNO, Quercianella, $0 \mathrm{~m}$, on fallow deer dung in d.c., F. Doveri, 19.12.12, 284.3-Rosignano Marittimo, CLSM 004.11 quater.

TOTAL 4: bird 1; cattle 1; fallow deer 1; roe deer 1.

Ascobolus stictoideus Speg., Michelia 1: 474, 1879.

Figs 109-112

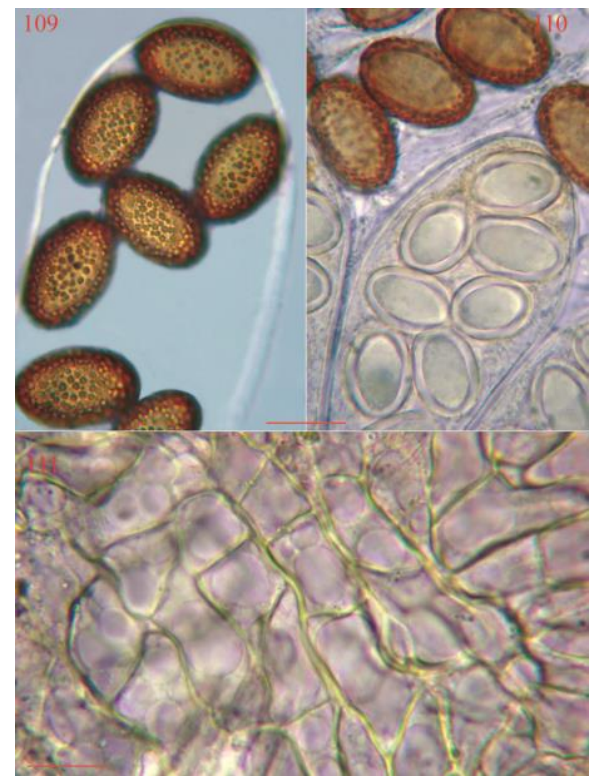

Figs 109-111 - Ascobolus stictoideus. 109-110 Asci with ascospores in different stages of maturity. 111 Detail of ectal excipulum. Bars $109-110=20 \mu \mathrm{m} .111=12 \mu \mathrm{m}$. 
Material examined - 1) FERRARA, S. Giustina wood (Mesola), $0 \mathrm{~m}$, dozens of gregarious specimens, almost fully immersed in horse dung, F. Bersan \& G. Visentin, 3.5.97, 187.1-Mesola, MCVE 580. 2) ROVIGO, Porto Caleri, $0 \mathrm{~m}$, on horse dung in d.c., F. Doveri, 5.97, 169.2Contarina, CLSM 00797 bis. 3) COSENZA, S. Demetrio Corone, $800 \mathrm{~m}$, on rat dung in d.c., C. Lavorato, 14.1.00, 552.4-S. Demetrio Corone, CLSM 00797 ter. 4) LIVORNO, Palazzi di Cecina, 0 m, on cattle dung, F. Doveri, 30.4.06, 294.1-Cecina, CLSM 00797 quater. 5) PISTOIA, Serravalle Pistoiese, 200 m, on duck dung, F. Doveri, 5.5.07, 262.1-Pistoia, CLSM 00797 penta.

TOTAL 5: horse 2; cattle 1; duck 1; rat 1.

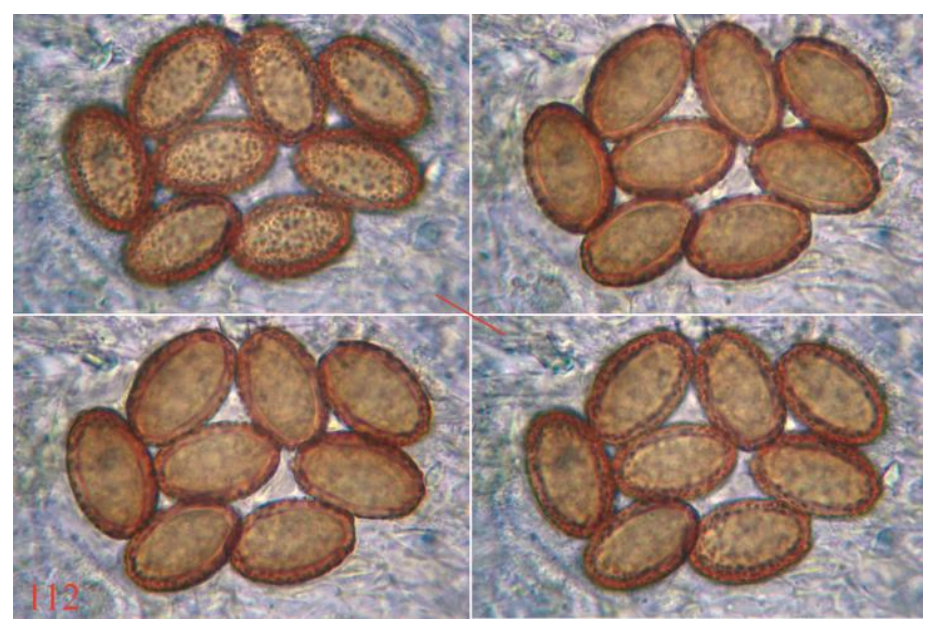

Fig 112 - Ascobolus stictoideus. Mature ascospores in different focuses. Bar $=20 \mu \mathrm{m}$.

Saccobolus beckii Heimerl, Jahresb. K. K. Ober-Realsch. Bez. Sechs. Wien 15: 18, 1889.

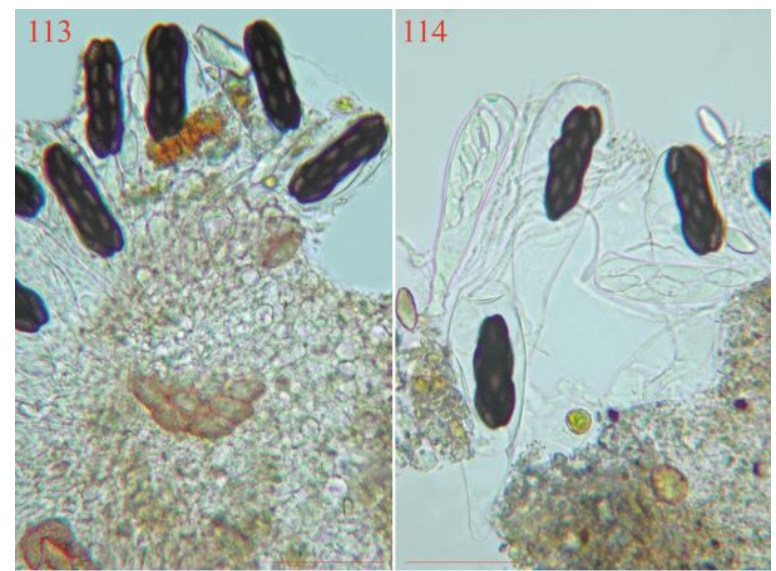

Figs 113-116

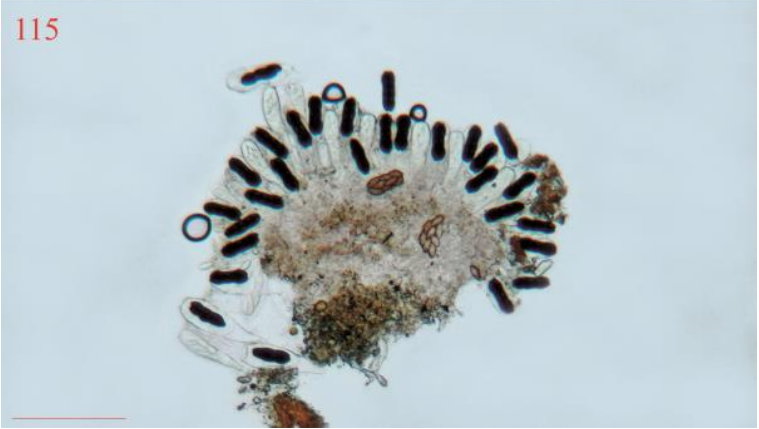

Figs 113-115 - Saccobolus beckii. 113-114 Details of longitudinal section of receptacle with asci containing spore clusters. 115 Longitudinal section of receptacle. Bars $113=60 \mu \mathrm{m} .114=20 \mu \mathrm{m}$. $115=150 \mu \mathrm{m}$. 
Material examined - 1) UDINE, Fleons gorge, $1400 \mathrm{~m}$, dozens of superficial, gregarious specimens on chamois dung in d.c., F. Bersan \& F. Doveri, 26.6.98, 031.1-Rigolato, CLSM 04898. 2) UDINE, Bruna Valley, $1700 \mathrm{~m}$, on deer dung, A. Bizzi, 28.6.98, 033.4-Malborghetto Valbruna, CLSM 04898 bis. 3) BELLUNO, Tambre d'Alpago, on deer dung in d.c., A. Bizzi, 18.7.98, 064.4Farra d'Alpago, CLSM 04898 ter. 4) FERRARA, Mesola, 0 m, on deer dung, A. Bizzi \& G. Zecchin, 17.4.99, 187.1-Mesola, CLSM 04898 quater. 5) TRENTO, Cort Montagne, $1100 \mathrm{~m}$, on roe deer dung, M. Castoldi \& G. Robich, 3.5.99, 059.3-Tione di Trento, CLSM 04898 penta. 6) BELLUNO, Tambre d'Alpago (loc. Pian Rosada), 1000 m, on deer dung, L. Levorato, 8.7.00, 064.4-Farra d'Alpago, CLSM 04898 esa. 7) BELLUNO, Tambre d'Alpago (loc. Pian Rosada), $1000 \mathrm{~m}$, on fallow deer dung in d.c., ?, 8.7.00, 064.4-Farra d'Alpago, CLSM 04898 epta. 8) BELLUNO, Croce d'Aune pass, $2000 \mathrm{~m}$, on roe deer dung, A. Bizzi, 22.6.03, 062.3-Fonzaso, CLSM 04898 octo. 9) BELLUNO, Tambre d'Alpago, $800 \mathrm{~m}$, on deer dung in d.c., G. Consiglio, 10.7.04, 064.4-Farra d'Alpago, CLSM 04898 ena. 10) BELLUNO, Lorenzago, $900 \mathrm{~m}$, on roe deer dung in d.c., A. Bizzi, 24.7.04, 030.3-Pieve di Cadore, CLSM 04898 deca. 11) BELLUNO, Tambre d'Alpago, Cansiglio Wood, loc. Pian Rosanda, 1000 m, on deer dung in d.c., L. Levorato, 16.7.05, 064.4-Farra d'Alpago, CLSM 04898-XI. 12) BELLUNO, Tambre d'Alpago, Cansiglio wood-Pian Rosanda, 1000 m, on deer dung in d.c., A. Bizzi, 16.7.05, 064.4-Farra d'Alpago, CLSM 04898-XII. 13) OGLIASTRA, Gairo Taquisara, Leperccei, $900 \mathrm{~m}$, on cattle dung in d.c., L. Arras, 2.12.06, 531.3-Ussàssai, CLSM 04898-XIII. 14) VICENZA, Lastebasse, Fiorentini, $1500 \mathrm{~m}$, on roe deer dung in d.c., A. Bizzi, 13.7.08, 081.1-Caldonazzo, CLSM 04898-XIV. 15) AOSTA, Salati Pass, $3000 \mathrm{~m}$, on chamois in d.c., L. Levorato, 28.8.08, 071.3-Gressoney la Trinité, CLSM 04898-XV. 16) BOLZANO, Aurina valley, $1300 \mathrm{~m}$, on deer dung in d.c., A. Bizzi, 16.7.11, 003.3-Valle Aurina, CLSM 04898-XVI.

TOTAL 16: deer 8; roe deer 4; chamois 2; cattle 1; fallow deer 1.

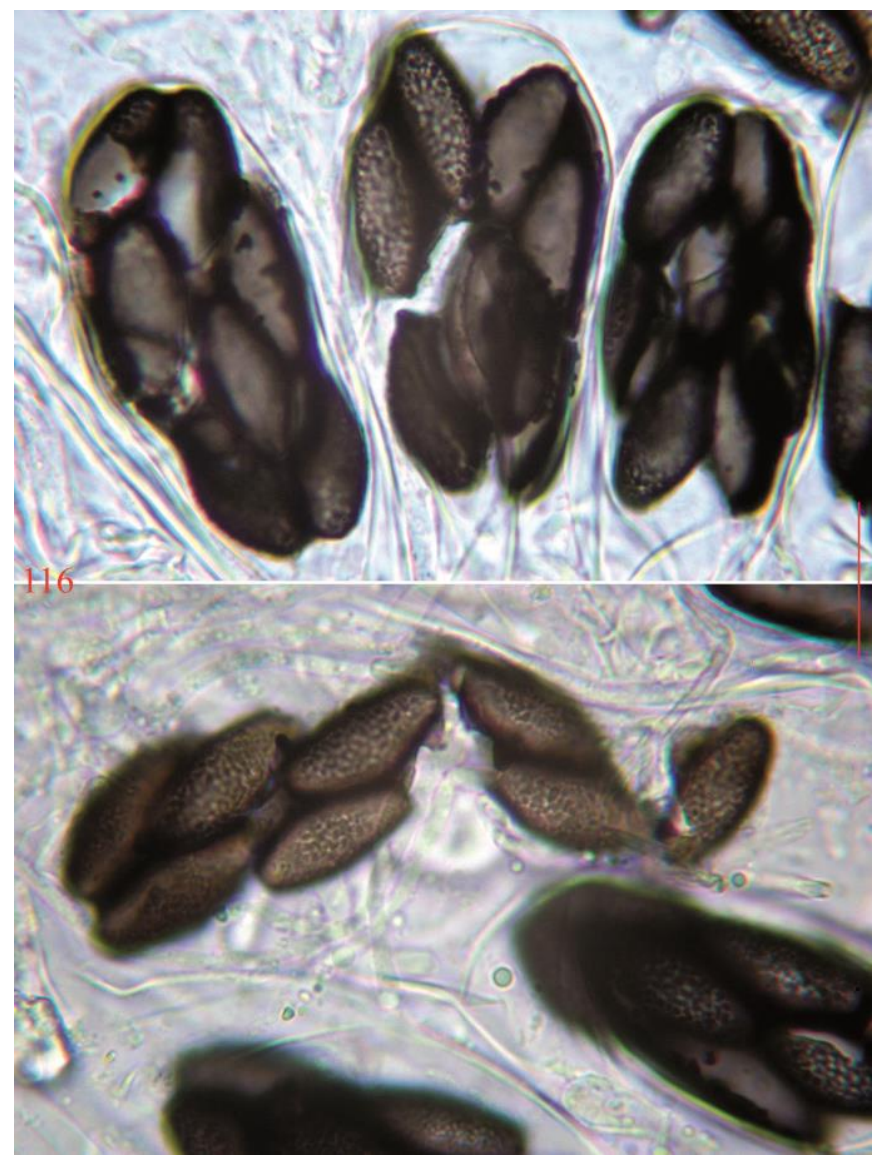

Fig 116 - Saccobolus beckii. Spore clusters. Bar $=15 \mu \mathrm{m}$. 


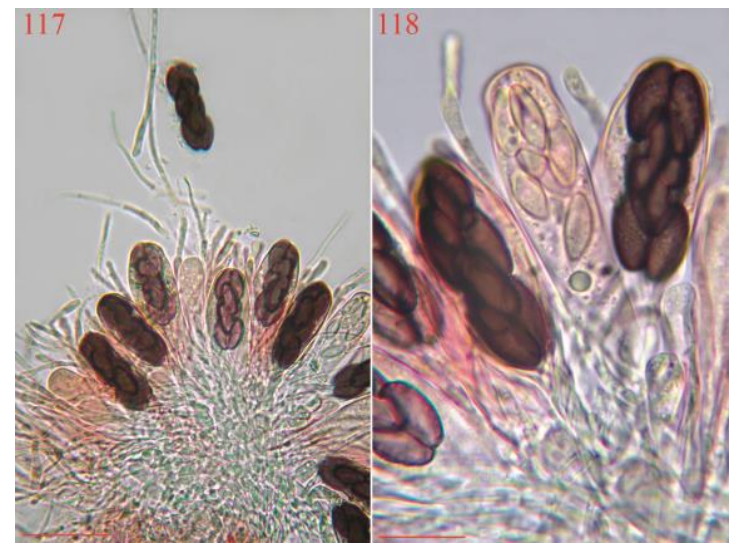

Figs 117-118 - Saccobolus caesariatus. 117 Hymenial surface. 118 Detail of hymenial surface with paraphyses and asci containing spore clusters. Bars $117=50 \mu \mathrm{m} .118=20 \mu \mathrm{m}$.

Material examined - 1) TRENTO, Forcella Juribrutto, $2400 \mathrm{~m}$, about fifty gregarious, superficial specimens, on sheep dung in d.c., E. Bizio, 28.7.99, 045.4-Soraga di Fassa, CLSM 02299. 2) AOSTA, Salati Pass, $3000 \mathrm{~m}$, on rock goat dung in d.c., L. Levorato, 24.8.00, 071.3Gressoney la Trinité, CLSM 02299 bis. 3) RAVENNA, Lido di Classe, 0 m, on wild rabbit dung in d.c., F. Doveri, 6.4.01, 241.3-Cervia, CLSM 02299 ter. 4) VENEZIA, Forte di S. Andrea, 0 m, on goat dung in d.c., E. Bizio, 3.6.01,128.3-Venezia, CLSM 02299 quater. 5) COSENZA, Mount Scuro, $1600 \mathrm{~m}$, on cattle dung in d.c., C. Lavorato, 4.9.01, 560.3-Spezzano della Sila, CLSM 02299 penta. 6) ROVIGO, Albarella isle, $0 \mathrm{~m}$, on wild rabbit dung, A. Bizzi, 16.11.02, 170.3-Porto Levante, CLSM 02299 esa. 7) VENEZIA, Caorle, loc. Brussa, 0 m, on wild rabbit dung in d.c., A. Bizzi, 10.10.04, 129.1-Caorle, CLSM 02299 epta. 8) L'Aquila, Gran Sasso, Campo Imperatore, $2400 \mathrm{~m}$, on sheep dung in d.c., L. Levorato, 11.5.07, 349.2-Gran Sasso d'Italia, CLSM 02299 octo. 9) TRENTO, Dimaro, Malga di Sadron, 1450 m, on cattle dung in d.c., F. Doveri, 8.9.07, 042.1Malé, CLSM 02299 ena. 10) VICENZA, Lastebasse, Fiorentini, 1500 m, on cattle dung in d.c., A. Bizzi, 13.7.08, 081.1-Caldonazzo, CLSM 02299 deca. 11) TRENTO, Vervò, Non valley, Predaia refuge, $1300 \mathrm{~m}$, on hare dung in d.c., A. Bizzi, 3.10.10, 043.4-Cles, CLSM 02299-XI.

TOTAL 11: cattle 3; wild rabbit 3; sheep 2; goat 1; hare 1; rock goat 1 .

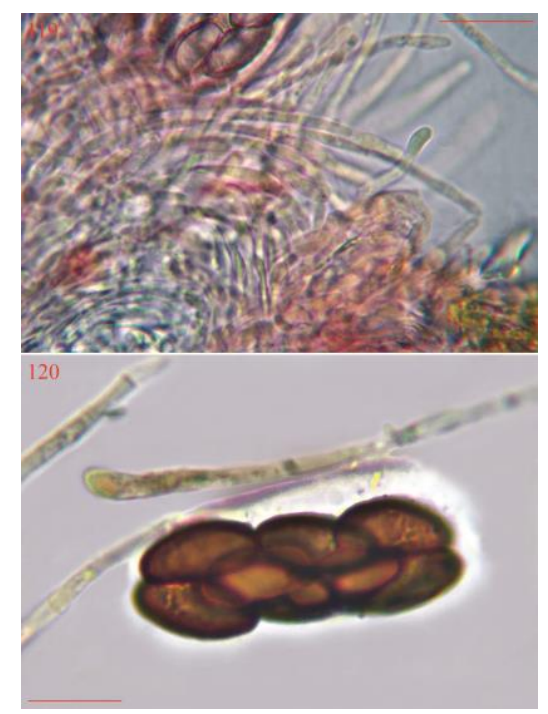

Figs 119-120 - Saccobolus caesariatus. 119 Hyphae at the base of receptacle. 120 Spore cluster and paraphyses. Bars $119=20 \mu \mathrm{m} .120=15 \mu \mathrm{m}$. 


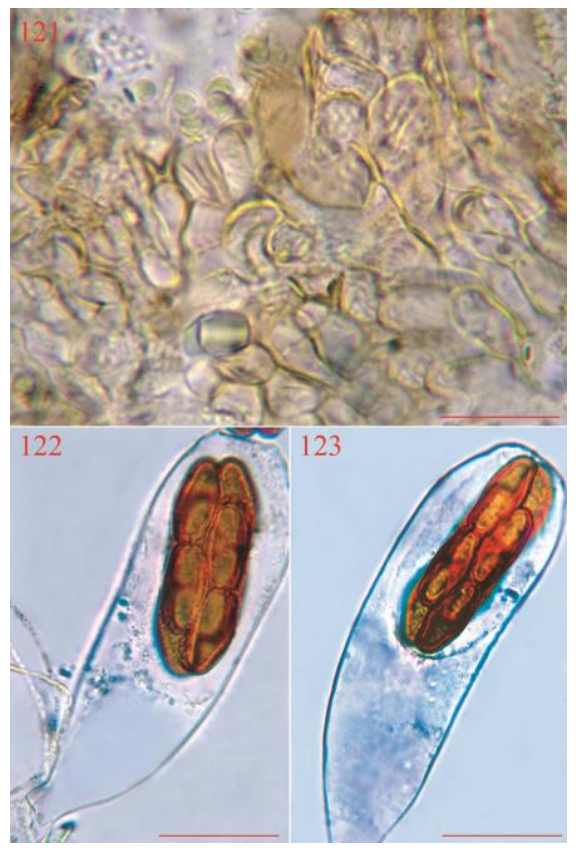

Figs 121-123 - Saccobolus citrinus. 121 Detail of ectal excipulum near the margin. 122-123 Asci with spore clusters. Bars $121=40 \mu \mathrm{m} .122-123=25 \mu \mathrm{m}$.

Material examined - 1) FERRARA, S. Giustina (Mesola), $0 \mathrm{~m}$, about ten gregarious, superficial specimens on deer dung in d.c., A. Bizzi \& G. Zecchin, 18.4.99, 187.1-Mesola, CLSM 01299. 2) ROVIGO, Albarella isle, $0 \mathrm{~m}$, on deer dung in d.c., G. Robich, 14.5.99, 170.3-Porto Levante. 3) VICENZA, Lugo, $350 \mathrm{~m}$, on horse dung in d.c., A. Bizzi, 29.10.99, 103.1-Marostica, CLSM 01299 quater. 4) PISA, S. Rossore park, 0 m, on fallow deer dung, F. Doveri, 5.3.00, 272.1Migliarino, CLSM 01299 penta. 5) VICENZA, Sarego (Mount Roccolo), $250 \mathrm{~m}$, on sheep dung in d.c., A. Bizzi, 9.00, 125.3-Montebello, CLSM 01299 esa. 6) MACERATA, Piobbico, 500 m, on cattle dung in d.c., F. Doveri, 2.5.02, 325.1-Bolognola, CLSM 01299 epta. 7) BELLUNO, Lorenzago,, $900 \mathrm{~m}$, on roe deer dung in d.c., A. Bizzi, 22.7.02, 030.3-Pieve di Cadore, CLSM 01299 octo. 8) ROVIGO, Albarella isle, 0 m, on roe deer dung in d.c., A. Bizzi, 16.11.02, 170.3Porto Levante, CLSM 01299 ena. 9) FERRARA, Mesola wood, 0 m, on roe deer dung in d.c., L. Levorato, 8.11.02, 187.1-Mesola, CLSM 01299 deca. 10) CAMPOBASSO, Vinchiaturo, $500 \mathrm{~m}$, on sheep dung in d.c., F. Doveri, 14.10.04, 405.2-Vinchiaturo, CLSM 01299-XI. 11) UDINE, Ampezzo, Pura pass, $1500 \mathrm{~m}$, on cattle dung in d.c., F. Bersan, 26.6.05, 031.3-Ampezzo, CLSM 01299-XII.

TOTAL 11: roe deer 3; cattle 2; deer 2; sheep 2; fallow deer 1; horse 1.

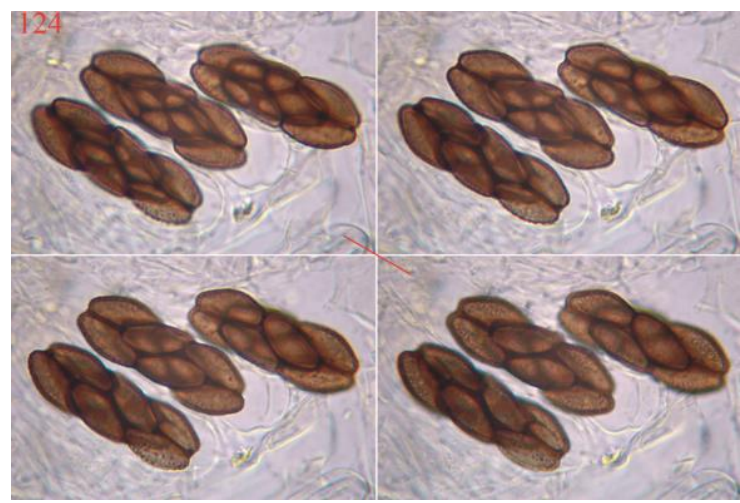

Fig 124 - Saccobolus citrinus. Spore clusters in four different focuses. Bar $=20 \mu \mathrm{m}$. 
Saccobolus depauperatus (Berk. \& Broome) E.C. Hansen, Vidensk. Meddel. Nathurist. Foren.: 87, 1876. Figs 125-128

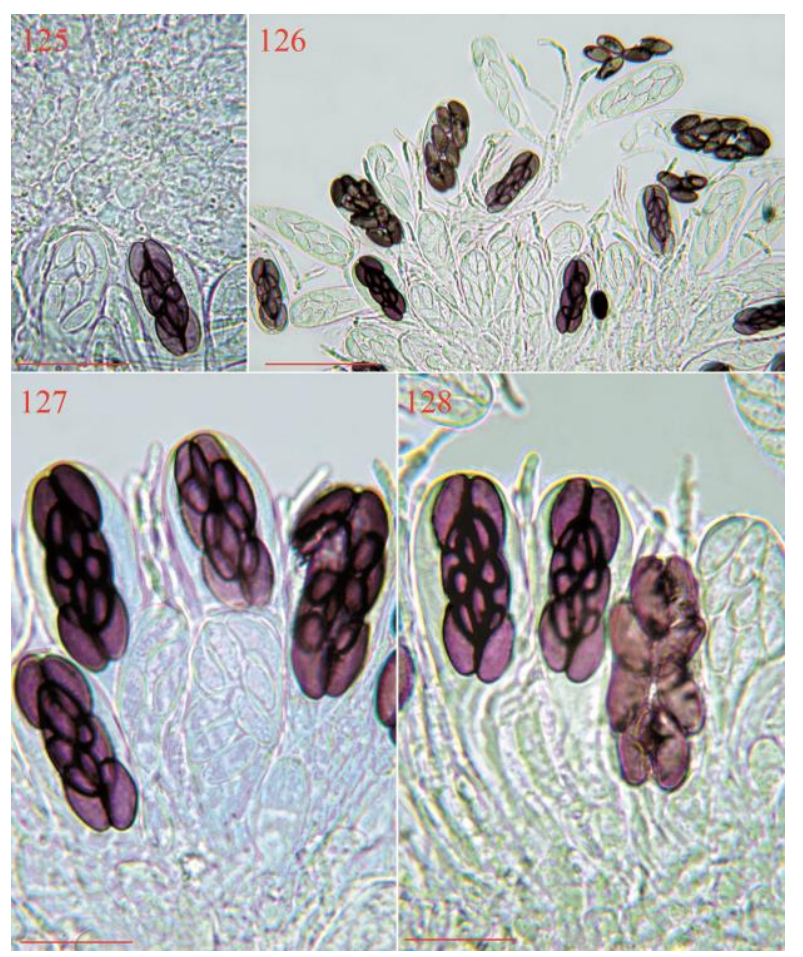

Figs 125-128 - Saccobolus depauperatus. 125 Detail of ectal excipulum and asci with spore clusters. 126 Detail of hymenial surface. 127-128 Paraphyses interspacing asci with spore clusters. Bars $125=30 \mu \mathrm{m} .126=50 \mu \mathrm{m} .127-128=20 \mu \mathrm{m}$.

Material examined - 1) PISA, Calambrone stables, $0 \mathrm{~m}$, dozens of gregarious specimens, many of which crowded, on horse dung in d.c., F. Doveri, 9.95, 272.2-Marina di Pisa, MCVE 492. 2) PISA, Calambrone stables, 0 m, on horse dung in d.c., F. Doveri, 28.11.95, 272.2-Marina di Pisa, CLSM 03195. 3) ROVIGO, Porto Caleri, $0 \mathrm{~m}$, on horse dung in d.c., F. Doveri, 5.97, 169.2Contarina, CLSM 03195 ter. 4) GORIZIA, Cona isle, 0 m, on horse dung in d.c., F. Bersan, 8.97, 109.1-Duino, AMB 6143 and CLSM 03195 quater. 5) TREVISO, Scalon, $200 \mathrm{~m}$, on sheep dung in d.c., E. Bizio, 6.6.97, 083.1-Valdobbiadene, CLSM 03195 penta. 6) BELLUNO, Forcella Negher, $2300 \mathrm{~m}$, on marmot dung in d.c., E. Bizio, 2.9.97, 045.1-Cencenighe Agordino, CLSM 03195 esa. 7) GROSSETO, Principina Terra, $0 \mathrm{~m}$, on horse dung in d.c., F. Doveri, 2.4.98, 331.3-Alberesese, CLSM 03195 epta. 8) UDINE, Fleons gorge, 1400 m, on deer dung in d.c., F. Bersan \& F. Doveri, 26.6.98, 031.1-Rigolato, CLSM 03195 octo. 9) TRENTO, Baselga di Piné, $1300 \mathrm{~m}$, on hare dung, A. Bizzi, 5.9.98, 060.1-Cembra, CLSM 03195 ena. 10) VICENZA, Nogarole Vicentino (Mount Faldo), 600 m, on horse dung in d.c., A. Bizzi, 1.9.99, 124.1-Arzignano, CLSM 03195 deca. 11) VICENZA, Lugo, $350 \mathrm{~m}$, on horse dung in d.c., A. Bizzi, 29.10.99, 103.1-Marostica, CLSM 03195XI. 12) VICENZA, Lugo, $350 \mathrm{~m}$, on horse dung in d.c., A. Bizzi, 30.10.99, 103.1-Marostica, CLSM 03195-XII. 13) VICENZA, Sarego (Mount Giaretta), 250 m, on horse dung in d.c., A. Bizzi, 10.00, 125.3-Montebello, CLSM 03195-XIII. 14) LECCE, Lizzanello stables, 40 m, on horse dung, V. Sciurti \& F. Doveri, 23.11.01, 512.1-Lecce, CLSM 03195-XIV. 15) VICENZA, Gallio-loc. Campomulo, $1400 \mathrm{~m}$, on horse dung in d.c., A. Bizzi, 16.8.01, 082.1-Monte Lisser, CLSM 03195XV. 16) VICENZA, Gallio-loc. Malga Molina, $1600 \mathrm{~m}$, on sheep dung in d.c., A. Bizzi, 16.8.01, 082.1-Monte Lisser, CLSM 03195-XVI. 17) COSENZA, Celico, $1200 \mathrm{~m}$, on horse dung in d.c., C. Lavorato, 4.9.01, 560.3-Spezzano della Sila, CLSM 03195-XVII. 18) VENEZIA, Alberoni, 0 m, on horse dung in d.c., A. Bizzi, 23.2.02, 148.1-Alberoni, CLSM 03195-XVIII. 19) TRENTO, Roncone, 850 m, on goat dung in d.c., F. Doveri, 11.9.02, 080.4-Roncone, CLSM 03195-XIX. 20) 
BELLUNO, Lorenzago, $900 \mathrm{~m}$, on roe deer dung in d.c., A. Bizzi, 22.7.02, 030.3-Pieve di Cadore, CLSM 03195-XX. 21) TRENTO, Regole di Malosco, $900 \mathrm{~m}$, on sheep (?) dung in d.c., G. Robich, 5.10.02, 026.3-Fondo, CLSM 03195-XXI. 22) ROVIGO, Albarella isle, $0 \mathrm{~m}$, on roe deer dung, A. Bizzi, 16.11.02, 170.3-Porto Levante, CLSM 03195-XXII. 23) BELLUNO, Lorenzago, $900 \mathrm{~m}$, on roe deer dung, L. Levorato, 23.3.03, 030.3-Pieve di Cadore, CLSM 03195-XXIII. 24) LECCO, Concenedo, $920 \mathrm{~m}$, on donkey dung in d.c., F. Doveri, 6.6.03, 076.4-Barzio, CLSM 03195-XXIV. 25) REGGIO CALABRIA, Tazza, $800 \mathrm{~m}$, on cattle dung in d.c., F. Doveri, 17.10.03, 602.3-Motta San Giovanni, CLSM 03195-XXV. 26) REGGIO CALABRIA, Passo Petrulli (Aspromonte), 1100 $\mathrm{m}$, on sheep dung in d.c., F. Doveri, 17.10.03, 602.4-Santo Stefano in Aspromonte, CLSM 03195XXVI. 27) VICENZA, Nagarole, Mount Faldo, 700 m, on hare dung in d.c., A. Bizzi, 20.4.04, 102.2-Valdagno, CLSM 03195-XXVII. 28) CAMPOBASSO, Campitello di Sepino, 1350 m, on horse dung in d.c., F. Doveri, 14.10.04, 405.2-Vinchiaturo, CLSM 03195-XXVIII. 29) CAMPOBASSO, Vinchiaturo, $500 \mathrm{~m}$, on sheep dung in d.c., F. Doveri, 14.10.04, 405.2Vinchiaturo, CLSM 03195-XXIX. 30) COSENZA, Celico, loc. Lagarò, $1200 \mathrm{~m}$, on horse dung in d.c., C. Lavorato, 21.3.05, 560.4-Celico, CLSM 03195-XXX. 31) GROSSETO, Follonica, 0 m, on fallow deer dung in d.c., F. Doveri, 5.5.2005, 318.4-Follonica, CLSM 03195-XXXI. 32) VICENZA, Barberino, S. Giovanni in Monte, ? m, on horse dung in d.c., A. Bizzi, 26.11.05, 125.2Longare, CLSM 03195-XXXII. 33) LIVORNO, Palazzi di Cecina, 0 m, on cattle dung, F. Doveri, 30.4.06, 294.1-Cecina, CLSM 03195-XXXIII. 34) FERRARA, Santa Giustina, Mesola wood, 0 m, on donkey dung in d.c., A. Bizzi, 10.11.05, 187.1-Mesola, CLSM 03195-XXXIV. 35) VICENZA, Valdagno, Zovo pass, $600 \mathrm{~m}$, on horse dung in d.c., A. Bizzi, 18.5.06, 102.2-Valdagno, CLSM 03195-XXXV. 36) FERRARA, Lido di Volano, Mesola wood, $0 \mathrm{~m}$, on horse dung in d.c., A. Bizzi, 18.5.06, 187.1-Mesola, CLSM 03195-XXXVI. 37) TRENTO, Dimaro, Malga di Sadron, $1450 \mathrm{~m}$, on cattle dung in d.c., F. Doveri, 8.9.07, 042.1-Malé, CLSM 03195-XXXVII. 38) TRENTO, Folgarida, Malga Folgarida, $1600 \mathrm{~m}$, on donkey dung in d.c., F. Doveri, 6.9.07, 042.2-Lago di Tovel, CLSM 03195-XXXVIII. 39) TRENTO, Vigo Rendena, $600 \mathrm{~m}$, on horse dung in d.c., F. Doveri, 6.9.07, 059.3-Tione di Trento, CLSM 03195-XXXIX. 40) VICENZA, Lonigo, 0 m, on horse dung in d.c., A. Bizzi, 19.8.07, 146.4-Lonigo, CLSM 03195-XL. 41) VICENZA, Gambugliano, $300 \mathrm{~m}$, on horse dung in d.c., A. Bizzi, 1.9.07, 125.4-Montebello Vicentino, CLSM 03195-XLI. 42) VENEZIA, Caorle, loc. Brussa, $0 \mathrm{~m}$, on horse dung in d.c., A. Bizzi, 27.10.07, 129.1-Caorle, CLSM 03195-XLII. 43) LIVORNO, Suvereto, La Suveraia farm, 0 m, on horse dung in d.c., F. Doveri, 18.3.09, 306.3-Suvereto, CLSM 03195-XLIII. 44) PISA, San Miniato Basso, 0 $\mathrm{m}$, on horse dung in d.c., F. Doveri, 13.4.09, 274.2-San Miniato, CLSM 03195-XLIV. 45) PISA, Volterra, Villamagna, Vallicella farm, $200 \mathrm{~m}$, on sheep dung in d.c., F. Doveri, 13.4.09, 285.3Lajatico, CLSM 03195-XLV. 46) PISA, Berignone wild park, $80 \mathrm{~m}$, on horse dung in d.c., F. Doveri, 6.9.09, 295.1-Mazzolla, CLSM 03195-XLVI. 47) PISA, Berignone wild park, $80 \mathrm{~m}$, on horse dung in d.c., F. Doveri, 6.9.09, 295.1-Mazzolla, CLSM 03195-XLVII. 48) NOVARA, Druogno, Santa Maria, $850 \mathrm{~m}$, on horse dung in d.c., F. Doveri, 15.5.10, 116.1-Bellinzago, CLSM 03195-XLVIII. 49) GROSSETO, Collacchia, $50 \mathrm{~m}$, on sheep dung in d.c., F. Doveri, 25.3.11, 319.4-Ribolla, CLSM 03195-XLIX. 50) GROSSETO, Monterotondo Marittimo, boraciferous lake, $250 \mathrm{~m}$, on horse dung in d.c., F. Doveri, 25.3.11, 306.4-Monterotondo Marittimo, CLSM 03195-L. 51) GROSSETO, Monterotondo Marittimo, boraciferous lake, $250 \mathrm{~m}$, on cattle dung in d.c., F. Doveri, 25.3.11, 306.4-Monterotondo Marittimo, CLSM 03195-LI. 52) LIVORNO, Elba island, ?, ?, on sheep dung in d.c., L. Levorato, 9.4.11, ?, CLSM 03195-LII. 53) LIVORNO, Elba island, ?, ?, on sheep dung in d.c., L. Levorato, 9.4.11, ?, CLSM 03195-LIII. 54) LIVORNO, Quercianella, $0 \mathrm{~m}$, on fallow deer dung in d.c., F. Doveri, 19.12.12, 284.3-Rosignano Marittimo, CLSM 03195-LIV. 55) LIVORNO, Quercianella, $0 \mathrm{~m}$, on fallow deer dung in d.c., F. Doveri, 19.12.12, 284.3-Rosignano Marittimo, CLSM 03195-LV. 56) LUCCA, Viareggio, $0 \mathrm{~m}$, on horse dung in d.c., G. Cacialli, 30.3.13, 260.2-Viareggio, CLSM 03195-LVI.

TOTAL 56: horse 29; sheep 9; cattle 4; donkey 3; fallow deer 3; roe deer 3; hare 2; deer 1; goat 1 ; marmot 1 . 


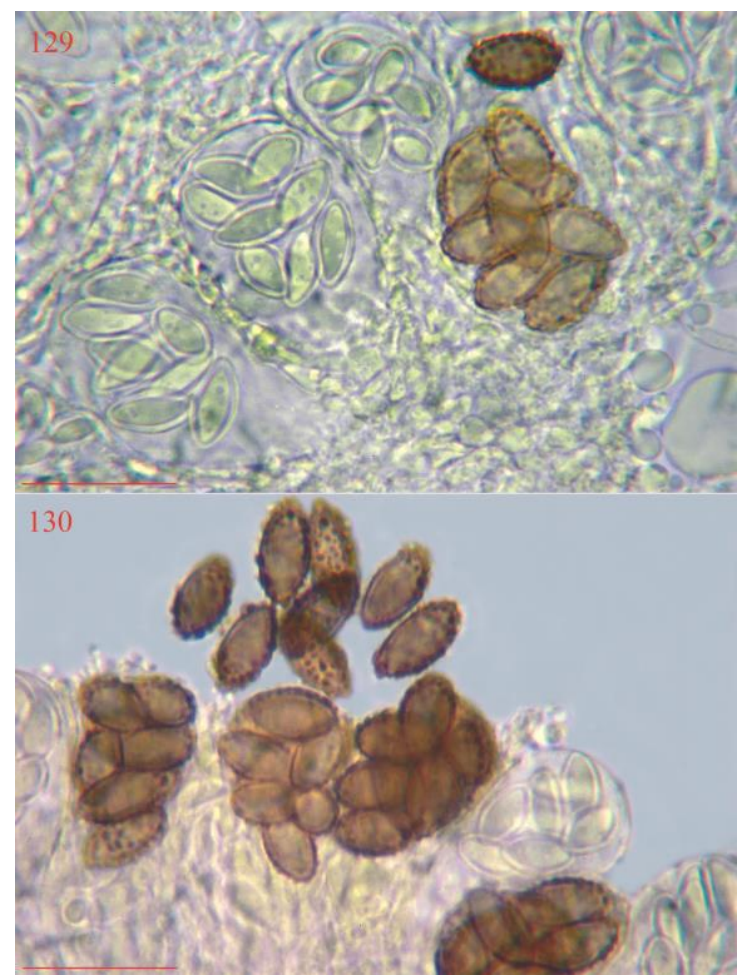

Figs 129-130 - Saccobolus dilutellus. 129 Longitudinal section of receptacle with cells of the ectal excipulum, immature asci, and a spore cluster. 130 Free ascospores above spore clusters. Bars $=20$ $\mu \mathrm{m}$.

Material examined - 1) ROVIGO, Porto Caleri, $0 \mathrm{~m}$, dozens of gregarious specimens on wild rabbit dung, E. Bizio, 22.11.97, 169.2-Contarina, CLSM 01098. 2) COSENZA, S. Demetrio Corone, $800 \mathrm{~m}$, on rat dung in d.c., C. Lavorato, 14.1.00, 552.4-S. Demetrio Corone, CLSM 01098 bis. 3) LIVORNO, Elba island, Campo nell'Elba, Prataccio, Valle Buia, $200 \mathrm{~m}$, on wild pig dung in d.c., C. Cotta, 15.10.10, 328.1-Marina di Campo, CLSM 01098 ter.

TOTAL 3: rat 1; wild pig 1; wild rabbit 1.

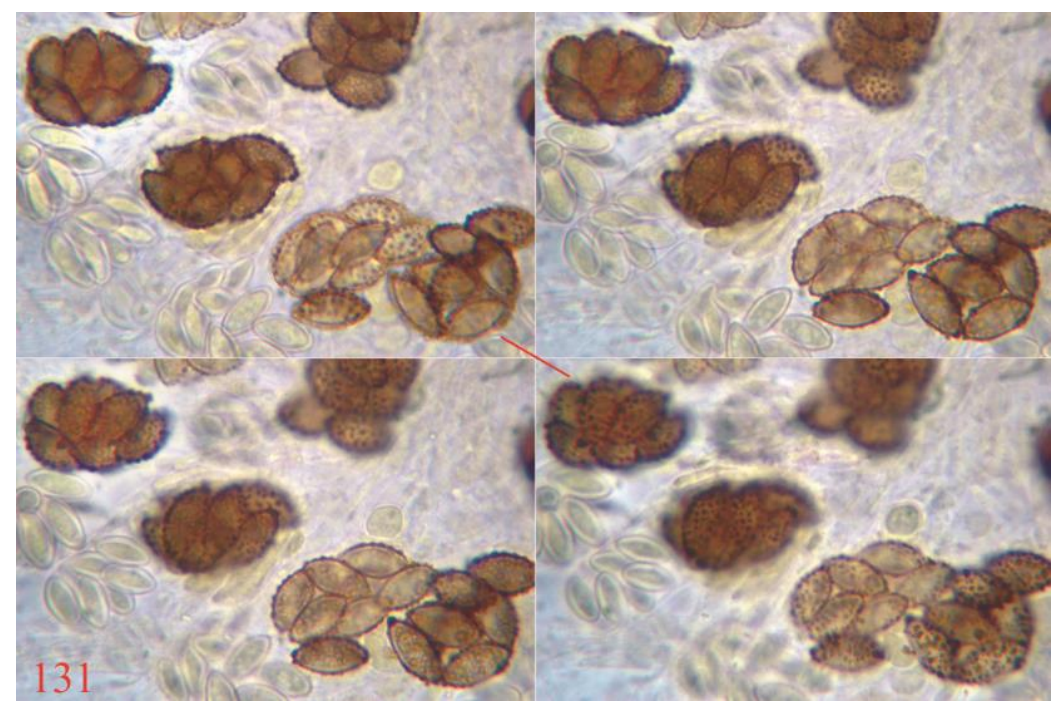

Fig 131 - Saccobolus dilutellus. Spore clusters in different focuses. Bar $=12 \mu \mathrm{m}$. 


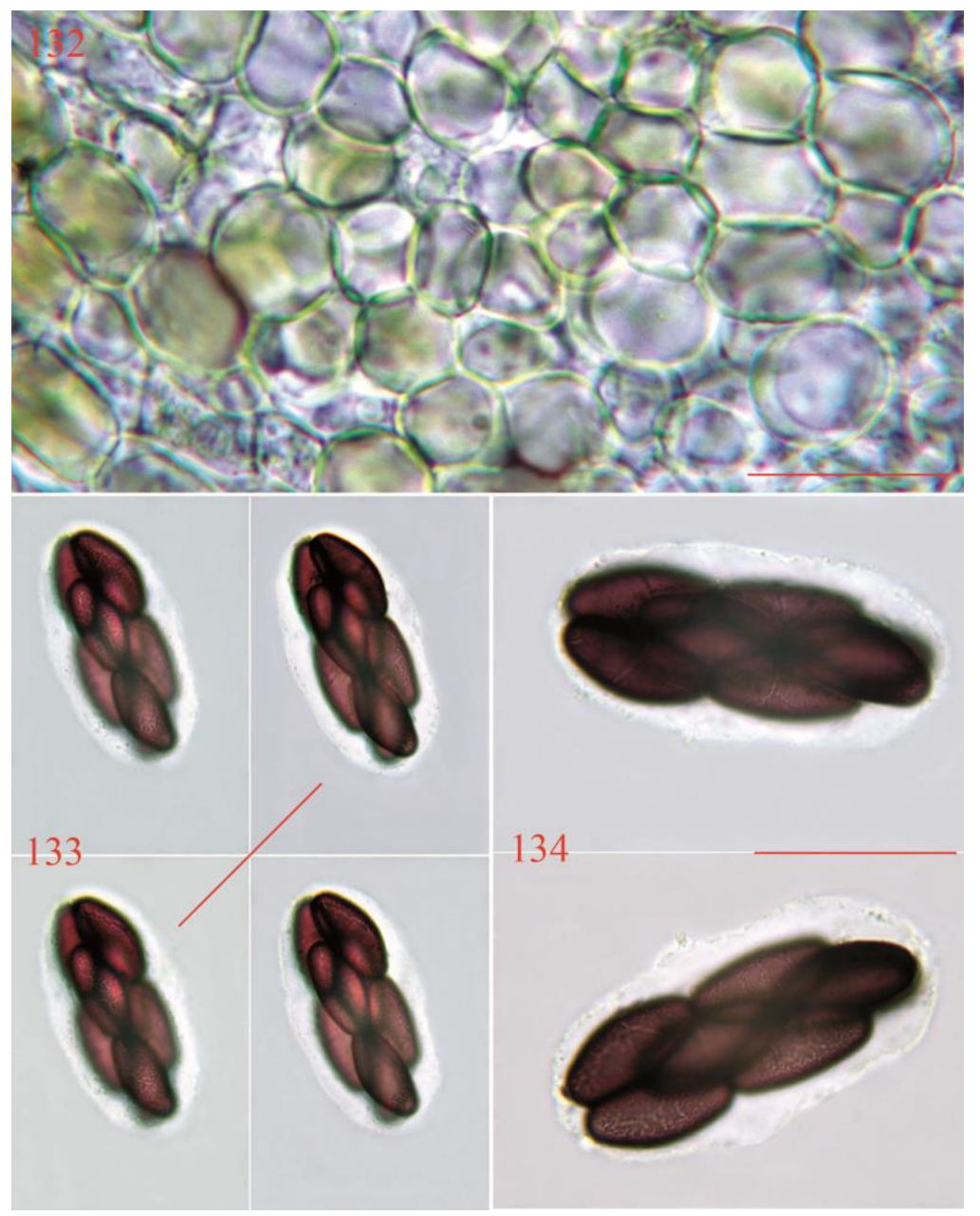

Figs 132-134 - Saccobolus glaber. 132 Detail of ectal excipulum. 133-134 Spore clusters in different focuses. Bars $132=12 \mu \mathrm{m} .133=60 \mu \mathrm{m} .134=35 \mu \mathrm{m}$.

Material examined - 1) BRESCIA: Lavenone, $400 \mathrm{~m}$, dozens of gregarious, superficial specimens, some of which crowded, on dung of unidentified herbivore, G. Medardi, 4.91, 100.4Vestone, MCVE-ERB2 10748. 2) COSENZA, San Demetrio Corone (Contrada Poggio), 600 m, on pig dung in d.c., C. Lavorato, 16.5.98, 552.4-San Demetrio Corone, CLSM 02596 ter. 3) PISA, Tirrenia farm, $0 \mathrm{~m}$, on cattle dung, F. Doveri, 13.5.00, 272.2-Marina di Pisa, CLSM 02596 quater. 4) VICENZA, Sarego (Mount Roccolo), 250 m, on sheep dung, A. Bizzi, 10.00, 125.3-Montebello, CLSM 02596 penta. 5) VERONA, S. Giorgio, $1500 \mathrm{~m}$, on cattle dung in d.c., A. Bizzi, 12.10.01, 102.3-Bosco Chiesanuova, CLSM 02596 esa. 6) PORDENONE, Pinedo Settimana valley, $1200 \mathrm{~m}$, on cattle dung in d.c., A. Bizzi, 27.7.03, 047.2-Claut, CLSM 02596 epta. 7) LIVORNO, Palazzi di Cecina, 0 m, on cattle dung, F. Doveri, 30.4.06, 294.1-Cecina, CLSM 02596 octo. 8) VICENZA, Recoaro Terme, Mount Rasta, 950 m, on sheep dung in d.c., F. Doveri, 8.6.07, 102.1-Recoaro Terme, CLSM 02596 ena. 9) VICENZA, Schio, S. Caterina di Tretto, $800 \mathrm{~m}$, on cattle dung in d.c., A. Bizzi, 1.9.07, 103.4-Malo, CLSM 02596 deca. 10) NOVARA, Druogno, Santa Maria, 850 m, on cattle dung in d.c., F. Doveri, 15.5.10, 116.1-Bellinzago, CLSM 02596-XI. 11) GROSSETO, Monterotondo Marittimo, Capannini, 100 m, on sheep dung in d.c., F. Doveri, 25.3.11, 306.4Monterotondo Marittimo, CLSM 02596-XII.

TOTAL 11: cattle 6; sheep 3; pig 1; unidentified herbivore 1. 


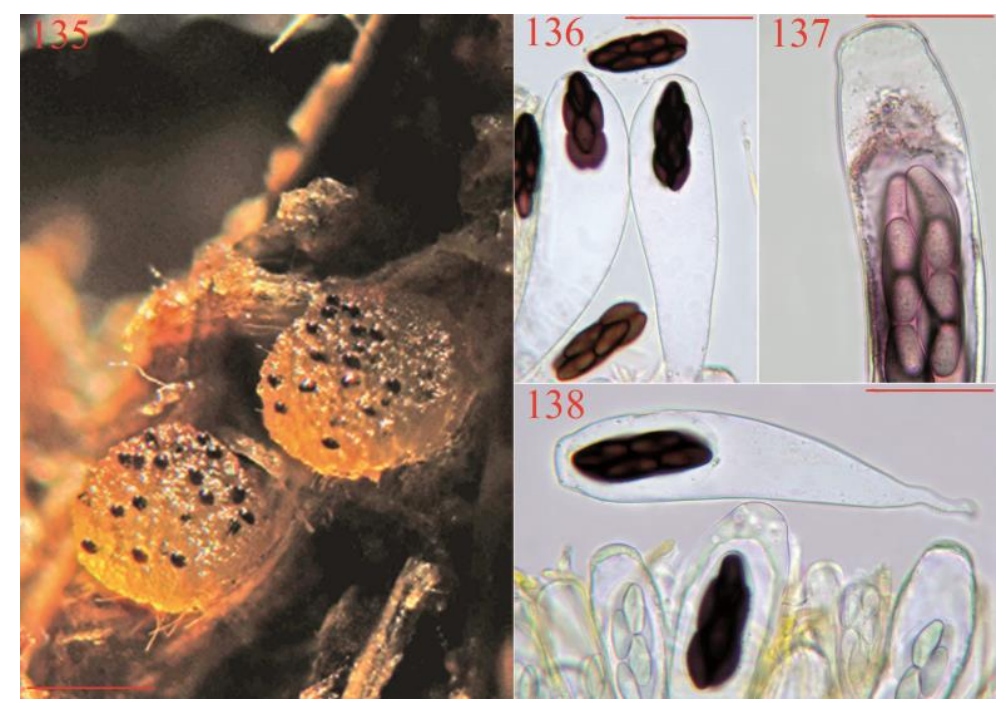

Figs 135-138 - Saccobolus glaber. 135 Ascomata on dung. 136-137 Asci with spore clusters. 138 Asci interspaced with paraphyses containing yellow pigments. Bars $135=400 \mu \mathrm{m} .136=70 \mu \mathrm{m}$. $137=35 \mu \mathrm{m} .138=50 \mu \mathrm{m}$.

Saccobolus minimus Velen., Monogr. Discom. Boh.: 370, 1934.

Figs 139-142

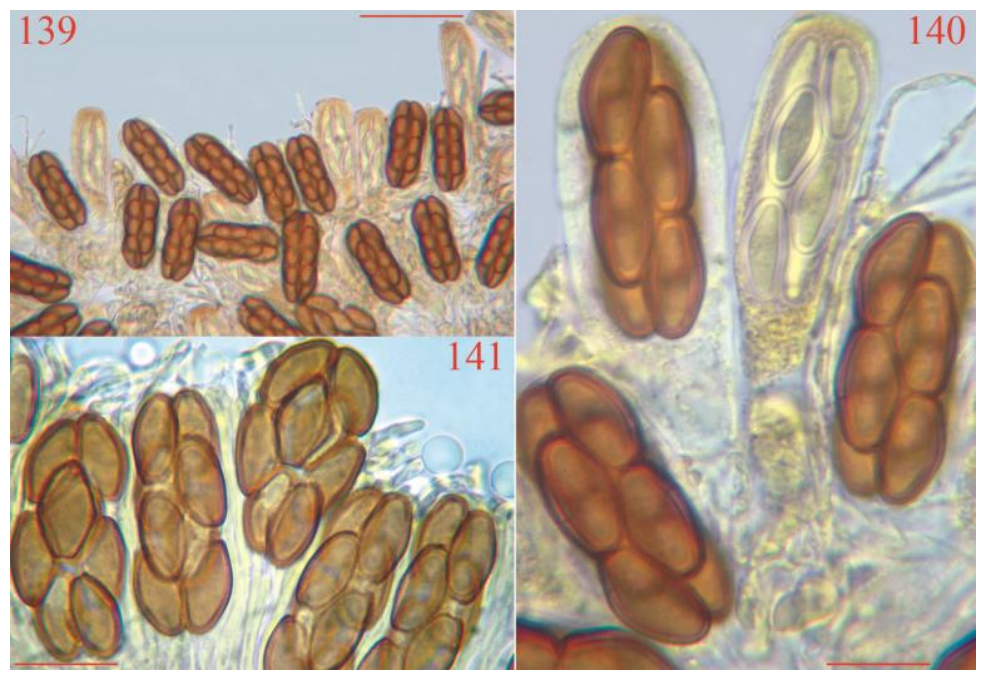

Figs 139-141 - Saccobolus minimus. 139 Detail of hymenial surface with numerous spore clusters. 140-141 Asci with spore clusters and exceeding paraphyses. Bars $139=45 \mu \mathrm{m} .140=12 \mu \mathrm{m} .141$ $=20 \mu \mathrm{m}$.

1) PISA, Calambrone stables, $0 \mathrm{~m}$, hundreds of gregarious specimens, many of which crowded, on horse dung in d.c., F. Doveri, 6.95, 272.2-Marina di Pisa, MCVE 491. 2) COSENZA, Corigliano Calabro, $300 \mathrm{~m}$, on goat dung in d.c., C. Lavorato, 16.5.98, 552.1-Corigliano Calabro, CLSM 02495 ter. 3) VICENZA, Sarego, 250 m, on horse dung in d.c., A. Bizzi, 20.8.99, 125.3Longare, CLSM 02495 quater. 4) VERCELLI, Mount Rosa (Gabiet lake), $2500 \mathrm{~m}$, on rock goat dung in d.c., G. Robich, 26.8.00, 071.2-Alagna Valsesia, 02495 penta. 5) VICENZA, Mount Caldiero, $230 \mathrm{~m}$, on sheep dung in d.c., A. Bizzi, 9.00, 125.3-Montebello, CLSM 02495 esa. 6) VENEZIA, Forte di S. Andrea, 0 m, on goat dung in d.c., E. Bizio, 3.6.01, 128.3-Venezia, CLSM 02495 epta. 7) LECCE, Vanze, $10 \mathrm{~m}$, on cattle dung in d.c., V. Sciurti \& F. Doveri, 24.11.01, 513.4-S. Foca, CLSM 02495 octo. 8) COSENZA, Acri-Contrada Mischinella, 1100 m, on wild pig dung in d.c., C. Lavorato, 19.10.01, 552.3-Acri, CLSM 02495 ena. 9) TRENTO, Regole di 
Malosco, 900 m, on sheep (?) dung in d.c., G. Robich, 5.10.02, 026.3-Fondo, CLSM 02495 deca. 10) RAVENNA, Ponte Bottole pinewood, $0 \mathrm{~m}$, on fallow deer dung in d.c., A. Bizzi, 11.11.02, 223.1-Marina Romea, CLSM 02495-XI. 11) PORDENONE, Pinedo (Val Settimana), 1200 m, on deer dung in d.c., A. Bizzi, 27.7.03, 047.2-Claut, CLSM 02495-XII. 12) Vicenza, Grancona, ? m, on sheep dung in d.c., A. Bizzi, 20.4.04, 125.3-Montebello Vicentino, CLSM 02495-XIV. 13) VICENZA, S. Zeno park, ? m, on roe deer dung in d.c., A. Bizzi, 6.11.03, 103.4-Schio, CLSM 02495-XV. 14) GROSSETO, Follonica, $0 \mathrm{~m}$, on fallow deer dung in d.c., F. Doveri, 5.5.2005, 318.4-Follonica, CLSM 02495-XVI. 15) BELLUNO, Lorenzago, Carera Rallo, 1600 m, on donkey dung, L. Levorato, 26.6.05, 030.3-Pieve di Cadore, CLSM 02495-XVII. 16) VICENZA, Barberino, S. Giovanni in Monte, ? m, on horse dung in d.c., A. Bizzi, 26.11.05, 125.2-Longare, CLSM 02495XVIII. 17) VICENZA, Grancona, Mount Caldiero, ? m, on sheep dung in d.c., A. Bizzi, 7.8.05, 125.3-Montebello Vicentino, CLSM 02495-XIX. 18) LIVORNO, Bibbona, La Pira farm holidays, $50 \mathrm{~m}$, on horse dung, F. Doveri, 21.5.06, 294.2-Bibbona, CLSM 02495-XX. 19) L'AQUILA, Celano, 800 m, on horse dung in d.c., F. Doveri, 22.6.06, 368.2-Celano, CLSM 02495-XXI. 20) VICENZA, Faldo, Mount Malo, 500 m, on horse dung in d.c., A. Bizzi, 2.12.06, 102.2-Valdagno, CLSM 02495-XXII. 21) VICENZA, Recoaro Terme, Mount Rasta, 950 m, on horse dung in d.c., F. Doveri, 8.6.07, 102.1-Recoaro Terme, CLSM 02495-XXIII. 22) VICENZA, Faldo, Mount Malo, $200 \mathrm{~m}$, on horse dung in d.c., A. Bizzi, 1.9.07, 102.2-Valdagno, CLSM 02495-XXIV. 23) LIVORNO, Elba island, ?, ?, on sheep dung in d.c., L. Levorato, 9.4.11, ?, CLSM 02495-XXV. 24) VENEZIA, Chioggia, Nordio wood, $0 \mathrm{~m}$, on roe deer dung in d.c., Cerello R. and Robich G., 27.4.13, 148.2-Chioggia, CLSM 02495-XXVI.

TOTAL 24: horse 8; sheep 5; goat 2; fallow deer 2; roe deer 2; cattle 1; deer 1; donkey 1; rock goat 1 ; wild pig 1 .

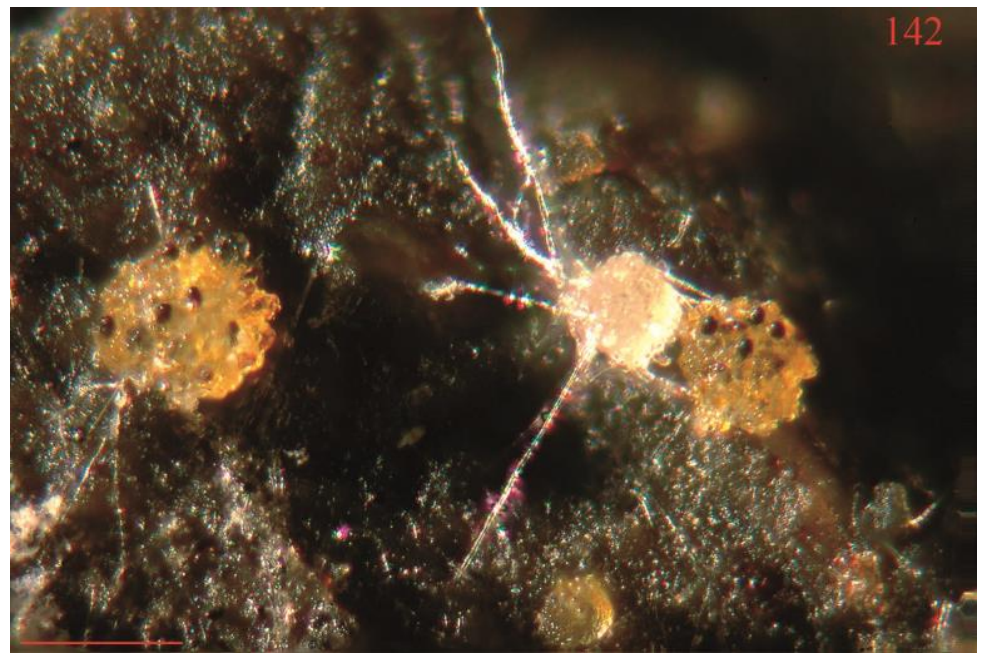

Fig 142 - Saccobolus minimus. Ascomata on dung. Bar $=300 \mu \mathrm{m}$.

Saccobolus obscurus

Figs $21-25$

TOTAL 3: fallow deer 1; sheep 1; wild rabbit 1.

Saccobolus saccoboloides (Seaver) Brumm., Persoonia suppl. 1: 168, 1967.

Figs 143-146

Material examined - 1) GROSSETO, Principina terra, $0 \mathrm{~m}$, eight isolated, superficial specimens, hidden in niches cattle dung in d.c., F. Doveri, 2.4.98, 331.3-Alberesese, CLSM 03298. 2) VICENZA, Gallio-loc. Malga Molina, $1600 \mathrm{~m}$, on horse dung in d.c., A. Bizzi, 16.8.01, 082.1Monte Lisser, CLSM 03298 bis. 3) TREVISO, Borso-loc. Mount Grappa, Campo Croce, 1200 m, on roe deer (?) dung in d.c., A. Bizzi, 10.8.01, 083.3-Monte Grappa, CLSM 03298 ter. 4) L'AQUILA, Celano, 800 m, on sheep dung in d.c., F. Doveri, 9.6.06, 368.2-Celano, CLSM 03298 penta. 


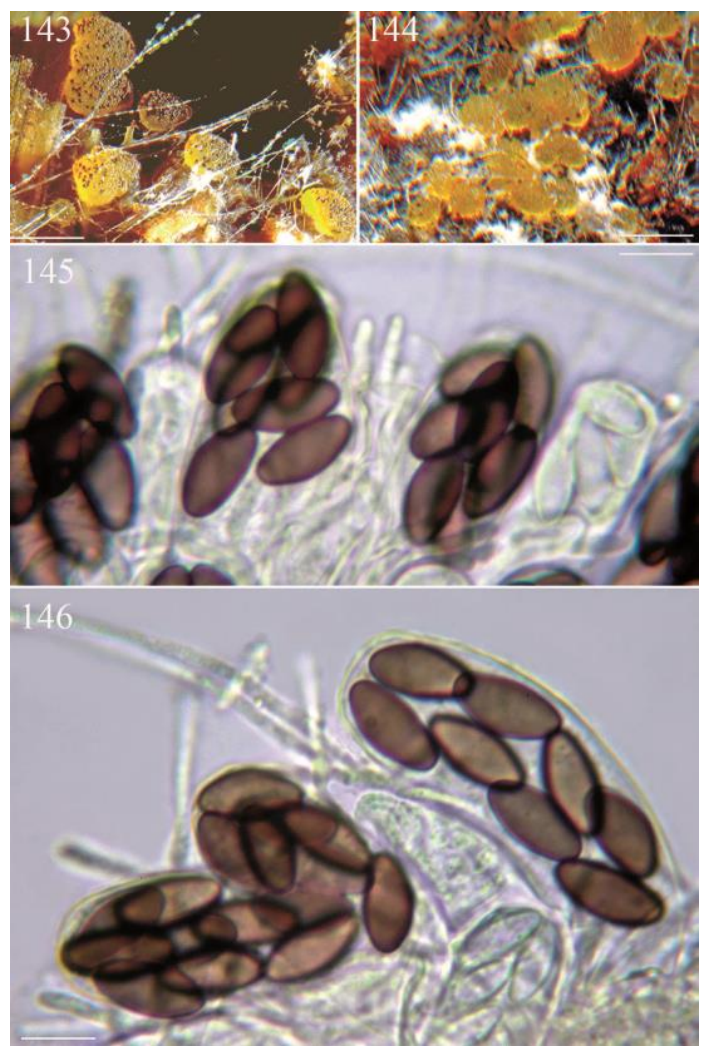

Figs 143-146 - Saccobolus saccoboloides. 143-144 Crowded ascomata on dung. 145-146 Asci with very loose spore clusters. Bars 143-144 = 250 $\mu \mathrm{m} .145-146=12 \mu \mathrm{m}$.

TOTAL 4: cattle 1; horse 1; roe deer 1; sheep 1.

Saccobolus succineus Brumm., Persoonia 5: 229, 1969.

Figs 147-153

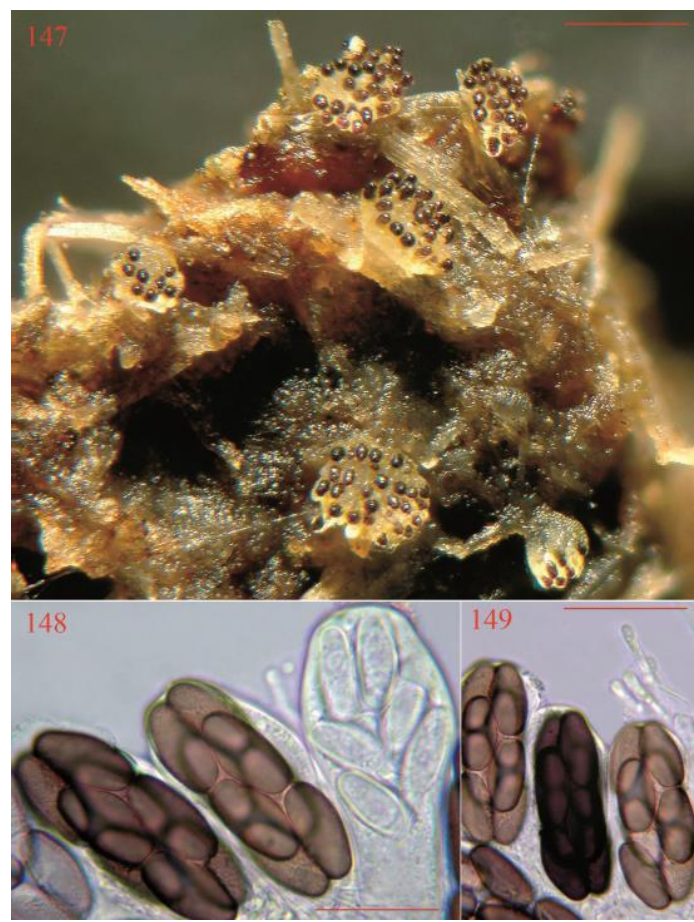

Figs 147-149 - Saccobolus succineus. 147 Ascomata on dung. 148-149 Asci with spore clusters. Bars $147=200 \mu \mathrm{m} .148=25 \mu \mathrm{m} .149=35 \mu \mathrm{m}$. 
Material examined - 1) LECCO, Cassina Valsassina, $850 \mathrm{~m}$, dozens of gregarious, superficial specimens on cattle dung in d.c., F. Doveri, 6.6.03, 076.4-Barzio, CLSM 012.03. 2) VERONA, Molina, $800 \mathrm{~m}$, on horse dung in d.c., A. Bizzi, 18.9.03, 101.2-Dolcè, CLSM 012.03 bis. 3) CAMPOBASSO, Campitello di Sepino, $1350 \mathrm{~m}$, on cattle dung in d.c., F. Doveri, 14.10.04, 405.2-Vinchiaturo, CLSM 012.03 ter. 4) CUNEO, Viola, loc. il Colletto, $1100 \mathrm{~m}$, on cattle dung, A. Bizzi, 14.9.05, 227.2-Pamperato, CLSM 012.03 quater. 5) VICENZA, Valli del Pasubio, Balasso refuge, $900 \mathrm{~m}$, on cattle dung in d.c., A. Bizzi, 14.5.06, 102.1-Recoaro Terme, CLSM 012.03 penta. 6) TRENTO, Folgaria, Forte Kerle, $1300 \mathrm{~m}$, on cattle dung in d.c., A. Bizzi, 12.10.06, 081.3Rovereto, CLSM 012.03 esa. 7) TRENTO, Dimaro, Malghetta, $1100 \mathrm{~m}$, on cattle dung in d.c., F. Doveri, 8.9.07, 042.1-Malé, CLSM 012.03 epta. 8) VICENZA, Faldo, Mount Malo, $200 \mathrm{~m}$, on horse dung in d.c., A. Bizzi, 1.9.07, 102.2-Valdagno, CLSM 012.03 octo. 9) LIVORNO, Montioni, $0 \mathrm{~m}$, on cattle dung in d.c., F. Doveri, 18.3.09, 306.3-Suvereto, CLSM 012.03 ena. 10) GROSSETO, Monterotondo Marittimo, boraciferous lake, $250 \mathrm{~m}$, on cattle dung in d.c., F. Doveri, 25.3.11, 306.4-Monterotondo Marittimo, CLSM 012.03 deca.

TOTAL 10: cattle 8; horse 2 .

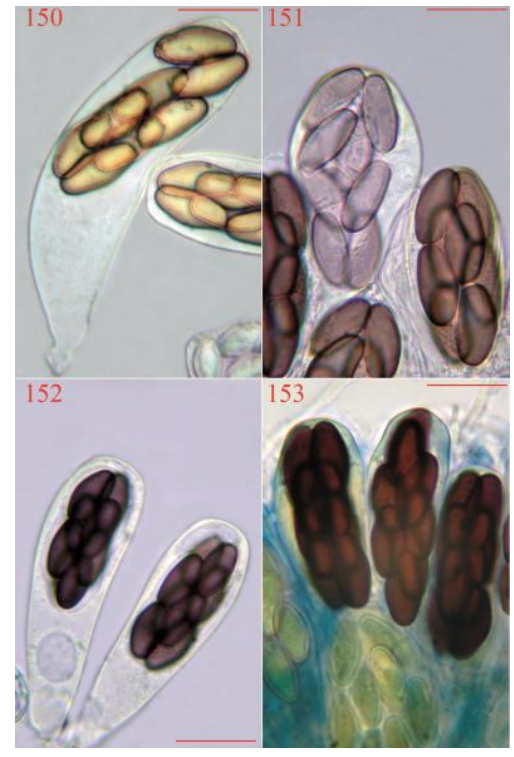

Figs 150-153 - Saccobolus succineus. 150-152 Asci with spore clusters. 153 Amyloid asci with spore clusters. Bars $150-151=20 \mu \mathrm{m} .152=25 \mu \mathrm{m} .153=22 \mu \mathrm{m}$.

Saccobolus truncatus Velen., Monogr. Discom. Boh. 1: 370, 1934.

Figs $154-158$

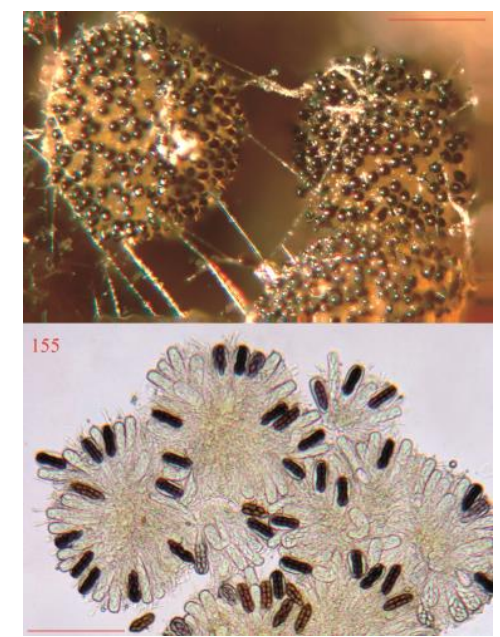

Figs 154-155 - Saccobolus truncatus. 154 Ascomata on dung. 155 Hymenial surface. Bars $154=$ $100 \mu \mathrm{m} .155=120 \mu \mathrm{m}$. 
Material examined - 1) LECCO, Vendrogno, $1190 \mathrm{~m}$, hundreds of gregarious or isolated, superficial specimens, on goat dung in d.c., A. Bizzi, 7.6.03, 055.3-Premana, CLSM 014.03. 2) REGGIO CALABRIA, Gambarie, $1300 \mathrm{~m}$, on cattle dung in d.c., A. Bizzi, 17.10.03, 602.1Gambarie, CLSM 014.03 bis. 3) COSENZA, S.Fili-loc. Carrera, ? m, on sheep dung in d.c., C. Lavorato, 16.11.04, 559.1-Rende, CLSM 014.03 ter. 4) LIVORNO, Bibbona, La Pira farm holidays, $50 \mathrm{~m}$, on horse dung, F. Doveri, 21.5.06, 294.2-Bibbona, CLSM 014.03 quater. 5) LIVORNO, Bibbona, La Pira farm holidays, $50 \mathrm{~m}$, on sheep dung, F. Doveri, 21.5.06, 294.2Bibbona, CLSM 014.03 penta. 6) L'AQUILA, Ovindoli, 1350 m, on cattle dung in d.c., F. Doveri, 9.6.06, 368.1-Ovindoli, CLSM 014.03 esa. 7) SIENA, Costafabbri, $150 \mathrm{~m}$, on horse dung in d.c., F. Doveri, 19.8.06, 296.1-Siena, CLSM 014.03 epta. 8) VICENZA, Valli del Pasubio, Balasso refuge, $900 \mathrm{~m}$, on cattle dung in d.c., A. Bizzi, 14.5.06, 102.1-Recoaro Terme, CLSM 014.03 octo. 9) VICENZA, Valdagno, Zovo pass, $600 \mathrm{~m}$, on horse dung in d.c., A. Bizzi, 18.5.06, 102.2-Valdagno, CLSM 014.03 ena. 10) VICENZA, Recoaro Terme, Mount Rasta, 950 m, on rabbit dung in d.c., F. Doveri, 8.6.07, 102.1-Recoaro Terme, CLSM 014.03 deca. 11) VICENZA, Gambugliano, 300 m, on horse dung in d.c., A. Bizzi, 1.9.07, 125.4-Montebello Vicentino, CLSM 014.03-XII. 12) PISA, Chiecinella, $70 \mathrm{~m}$, on horse dung in d.c., F. Doveri, 13.4.09, 274.3-Montopoli in Val d'Arno, CLSM 014.03-XIII. 13) PISA, Berignone wild park, $80 \mathrm{~m}$, on horse dung in d.c., F. Doveri, 6.9.09, 295.1-Mazzolla, CLSM 014.03-XIV. 14) PISA, Berignone wild park, $80 \mathrm{~m}$, on horse dung in d.c., F. Doveri, 6.9.09, 295.1-Mazzolla, CLSM 014.03-XV. 15) LIVORNO, Elba island, Campo nell'Elba, Prataccio, Valle Buia, 200 m, on wild pig dung in d.c., C. Cotta, 15.10.10, 328.1-Marina di Campo, CLSM 014.03-XVI. 16) GROSSETO, Monterotondo Marittimo, boraciferous lake, 250 $\mathrm{m}$, on cattle dung in d.c., F. Doveri, 25.3.11, 306.4-Monterotondo Marittimo, CLSM 014.03-XVII. 17) GROSSETO, Gavorrano rail-way station, $70 \mathrm{~m}$, on cattle dung in d.c., F. Doveri, 25.3.11, 318.1-Gavorrano, CLSM 014.03-XVIII. 18) GROSSETO, Gavorrano rail-way station, $70 \mathrm{~m}$, on horse dung in d.c., F. Doveri, 25.3.11, 318.1-Gavorrano, CLSM 014.03-XIX. 19) GROSSETO, Monterotondo Marittimo, boraciferous lake, $250 \mathrm{~m}$, on cattle dung in d.c., F. Doveri, 25.3.11, 306.4-Monterotondo Marittimo, CLSM 014.03-XX. 20) LIVORNO, Elba island, ?, ?, on sheep dung in d.c., L. Levorato, 9.4.11, ?, CLSM 014.03-XXI.

TOTAL 20: horse 8; cattle 6; sheep 3; goat 1; rabbit 1; wild pig 1.

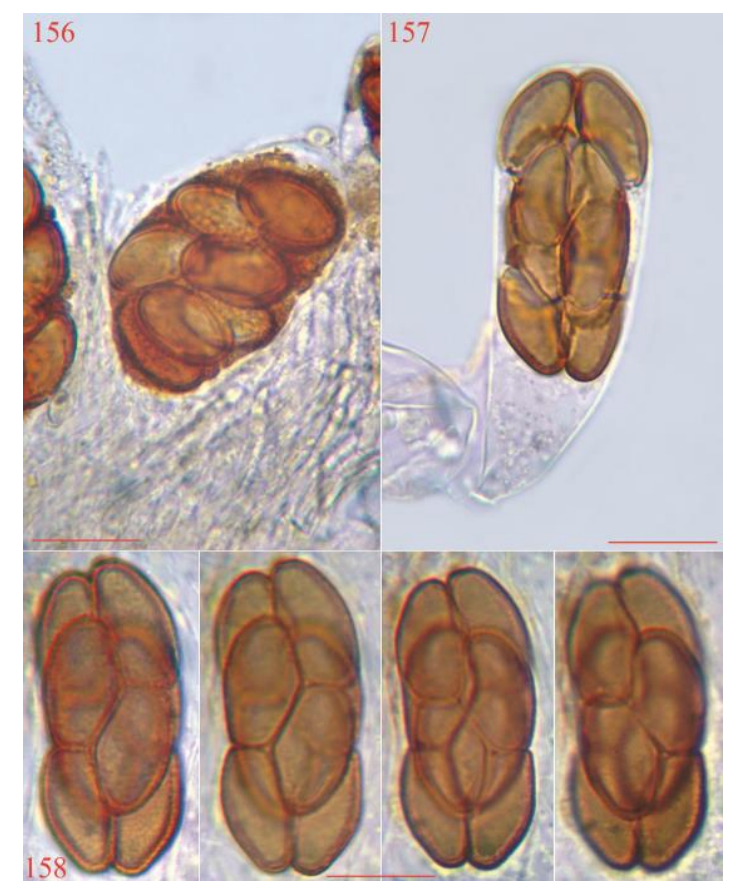

Figs 156-158 - Saccobolus truncatus. 156 Contracted spore cluster. 157 Ascus with a spore cluster. 158 Spore cluster in four different focuses. Bars 156-157 $=13 \mu \mathrm{m} .158=12 \mu \mathrm{m}$. 


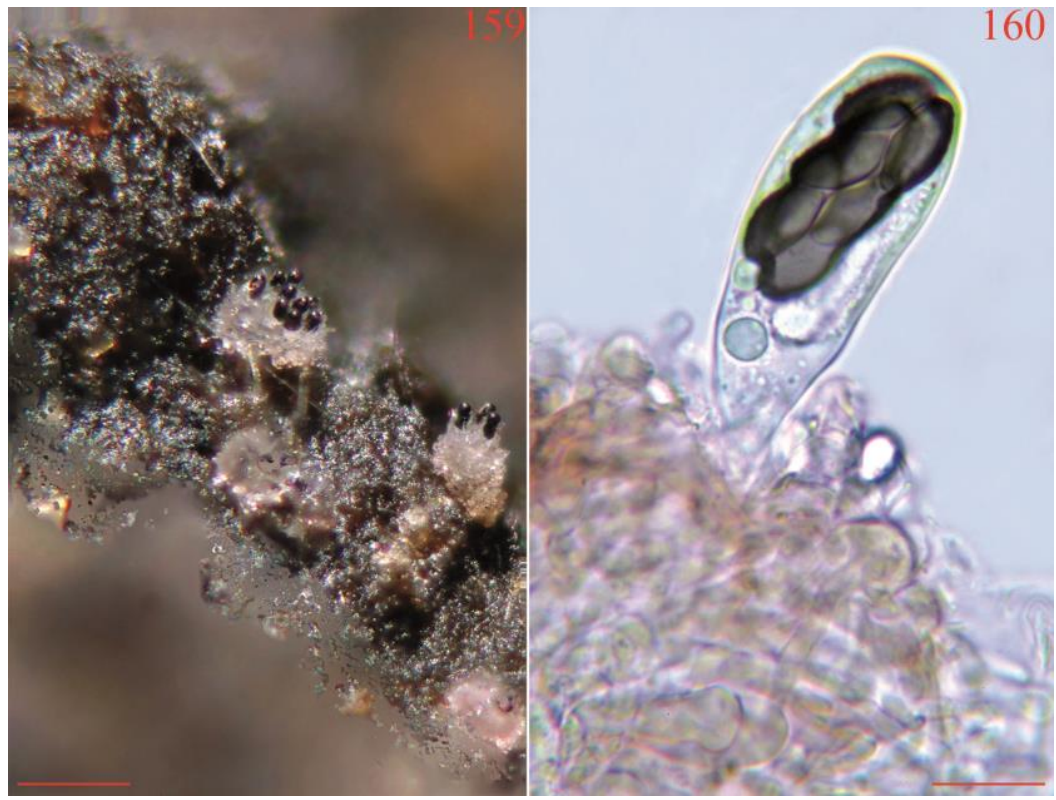

Figs 159-160 - Saccobolus verrucisporus. 159 Ascomata on dung. 160 Ascus with a spore cluster above violet pigmented cells of ectal excipulum. Bars $159=200 \mu \mathrm{m} .160=20 \mu \mathrm{m}$.

Material examined - 1) BELLUNO, Tambre d'Alpago, Cansiglio Wood (loc. Campon), $1000 \mathrm{~m}$, ten gregarious, superficial specimens, on cervine dung, A. Bizzi, 17.7.99, 064.4-Farra d'Alpago, CLSM 02799. 2) TRENTO, Centa S. Nicolò (loc. Frisanco), 1100 m, on roe deer dung in d.c., ?, 24.8.00, 081.1-Caldonazzo, CLSM 02799 bis. 3) COSENZA, S. Giovanni in Fiore-loc. Pettinascura, $1600 \mathrm{~m}$, on roe deer dung in d.c., C. Lavorato, 26.2.01, 560.1-Monte Volpintesta, CLSM 02799 ter. 4) TRENTO, Centa S. Nicolò-Palude refuge, 1000 m, on roe deer dung in d.c., A. Bizzi, 1.9.01, 081.1-Caldonazzo, CLSM 02799 quater. 5) VICENZA, Posina, ?, on roe deer dung in d.c., A. Bizzi, 25.5.02, 081.2-Castana, CLSM 02799 penta. 6) BELLUNO, Lorenzago, 900 m, on roe deer dung in d.c., L. Levorato, 23.3.03, 030.3-Pieve di Cadore, CLSM 02799 esa. 7) PORDENONE, Pinedo (Val Settimana), $1200 \mathrm{~m}$, on deer dung, A. Bizzi, 27.7.03, 047.2-Claut, CLSM 02799 epta. 8) BELLUNO, Campon-Farra d'Alpago, 1000 m, on deer dung in d.c., A. Bizzi, 5.7.03, 064.4-Farra d'Alpago, CLSM 02799 octo. 9) BELLUNO, Croce d'Aune pass, $2000 \mathrm{~m}$, on sheep dung in d.c., A. Bizzi, 22.6.03, 062.3-Fonzaso, CLSM 02799 ena. 10) BELLUNO, CamponFarra d'Alpago, $1000 \mathrm{~m}$, on horse dung in d.c., A. Bizzi, 5.7.03, 064.4-Farra d'Alpago, CLSM 02799 deca. 11) PORDENONE, Pinedo (Val Settimana), $1300 \mathrm{~m}$, on roe deer dung in d.c., A. Bizzi, 27.7.03, 047.2-Claut, CLSM 02799-XI. 12) PORDENONE, Pinedo (Val Settimana), $1300 \mathrm{~m}$, on deer dung, A. Bizzi, 27.7.03, 047.2-Claut, CLSM 02799-XII. 13) BELLUNO, Tambre d'Alpago, $800 \mathrm{~m}$, on deer dung in d.c., G. Consiglio, 10.7.04, 064.4-Farra d'Alpago, CLSM 02799-XIII. 14) BELLUNO, Lorenzago, $900 \mathrm{~m}$, on roe deer dung in d.c., A. Bizzi, 24.7.04, 030.3-Pieve di Cadore, CLSM 02799-XIV. 15) BELLUNO, Tambre d'Alpago, $800 \mathrm{~m}$, on deer dung in d.c., A. Bizzi, 10.7.04, 064.4-Farra d'Alpago, CLSM 02799-XV. 16) VICENZA, Pauna, $600 \mathrm{~m}$, on roe deer dung in d.c., A. Bizzi, 18.7.04, 102.1-Recoaro Terme, CLSM 02799-XVI. 17) BELLUNO, Tambre d'Alpago, Cansiglio wood-Pian Rosanda, 1000 m, on deer dung in d.c., A. Bizzi, 16.7.05, 064.4Farra d'Alpago, CLSM 02799-XVII. 18) BELLUNO, Tambre d'Alpago, Cansiglio wood-Pian Rosanda, $1000 \mathrm{~m}$, on deer dung in d.c., A. Bizzi, 16.7.05, 064.4-Farra d'Alpago, CLSM 02799XVIII. 19) L'Aquila, Gran Sasso, Campo Imperatore, $2400 \mathrm{~m}$, on roe deer dung in d.c., L. Levorato, 11.5.07, 349.2-Gran Sasso d'Italia, CLSM 02799-XIX. 20) TRENTO, Tres, Mount Corno, $1600 \mathrm{~m}$, on roe deer dung in d.c., A. Bizzi, 4.10.08, 043.4-Cles, CLSM 02799-XX. 21) VICENZA, Lastebasse, Fiorentini, $1500 \mathrm{~m}$, on roe deer dung in d.c., A. Bizzi, 13.7.08, 081.1- 
Caldonazzo, CLSM 02799-XXI. 22) VICENZA, Lastebasse, Fiorentini, $1500 \mathrm{~m}$, on roe deer dung, A. Bizzi, 13.7.08, 081.1-Caldonazzo, CLSM 02799-XXII. 23) LIVORNO, Elba island, Campo nell'Elba, Prataccio, Valle Buia, 200 m, on mouflon dung in d.c., C. Cotta, 15.10.10, 328.1-Marina di Campo, CLSM 02799-XXIII. 24) UDINE, Forni di Sopra, $1400 \mathrm{~m}$, on deer dung in d.c., A. Bizzi, 15.9.11, 030.2-Forni di Sopra, CLSM 02799-XXIV. 25) BOLZANO, Aurina valley, $1300 \mathrm{~m}$, on deer dung in d.c., A. Bizzi, 16.7.11, 003.3-Valle Aurina, CLSM 02799-XXV. 26) GROSSETO, Giglio island, Castello, 400 m, on wild rabbit dung in d.c., S. Rum, 22.3.12, 352.4-Giglio, CLSM 02799-XXVI. 27) POTENZA, Satriano Moliterno, $600 \mathrm{~m}$, on cattle dung in d.c., L. Levorato, 26.5.12, 488.2-Brienza, CLSM 02799-XXVI.

TOTAL 27: roe deer 12; deer 10; cattle 1; horse 1; mouflon 1; sheep 1; wild rabbit 1 .

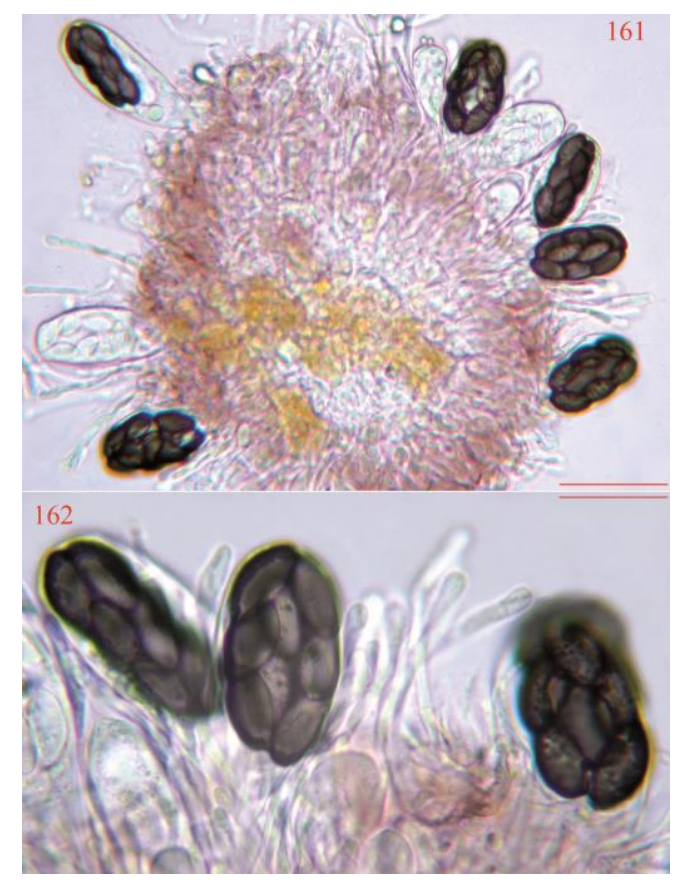

Figs 161-162 - Saccobolus verrucisporus. 161 Transverse section of receptacle. 162 Asci with spore clusters and apices of paraphyses. Bars $161=35 \mu \mathrm{m} .162=20 \mu \mathrm{m}$.

Saccobolus versicolor (P. Karst.) P. Karst., Acta Soc. Fauna Fl. Fenn. 2 (6): 123, 1885. Figs 163-165

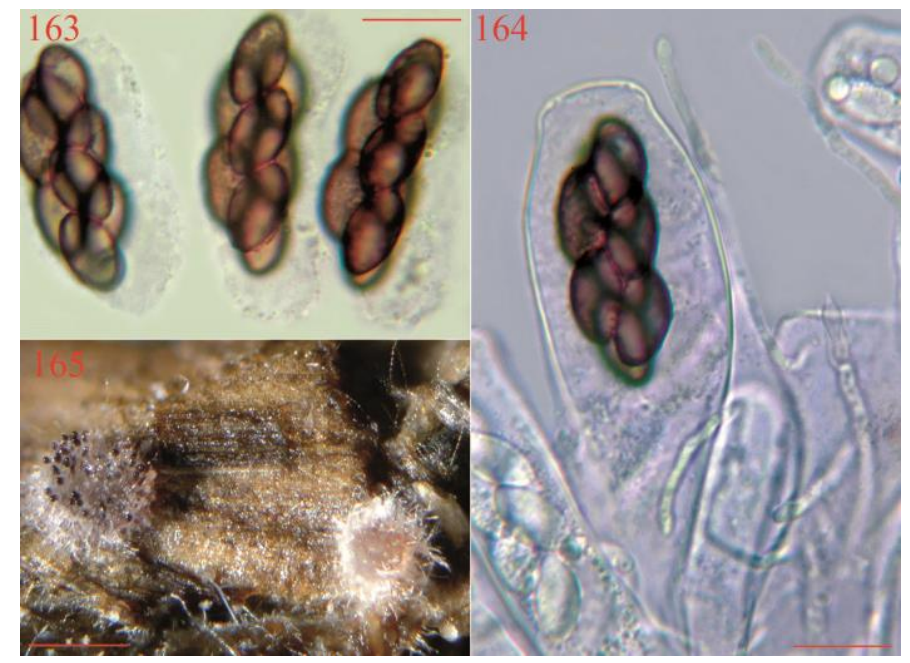

Figs 163-165 - Saccobolus versicolor. 163 Spore clusters. 164 Ascus with spore cluster and apices of paraphyses. 165 Ascomata on dung. Bars 163-164 $=20 \mu \mathrm{m} .165=500 \mu \mathrm{m}$. 
Material examined - 1) TRENTO, Brenta Valley, $1000 \mathrm{~m}$, dozens of gregarious specimens on cattle dung, G. Medardi, 5.96, 042.3-Madonna di Campiglio, MCVE 578. 2) VENEZIA, Alberoni, $0 \mathrm{~m}$, on wild rabbit dung in d.c., E. Bizio, 5.97, 148.1-Alberoni, CLSM 04696 bis. 3) VENEZIA, Caroman, 0 m, on wild rabbit dung in d.c., F. Doveri, 5.97, 148.2-Chioggia, CLSM 04696 ter. 4) TRENTO, Panarotta refuge, $1800 \mathrm{~m}$, on cattle dung in d.c., A. De Vito, 25.9.97, 060.2-Pergine, CLSM 04696 quater. 5) COSENZA, Orsomarso, $1300 \mathrm{~m}$, on donkey dung in d.c., C. Lavorato, 17.5.98, 533.2-Mormanno, CLSM 04696 penta. 6) UDINE, Mount Crostis, $2000 \mathrm{~m}$, on cattle dung in d.c., F. Bersan, 26.6.98, 031.1-Rigolato, CLSM 04696 esa. 7) COSENZA, Fossiata, $1400 \mathrm{~m}$, on wolf dung in d.c., C. Lavorato, 22.5.98, 560.1-Monte Volpintesta, CLSM 04696 epta. 8) CATANIA, Etna park, 1200 m, on wild rabbit dung, G. Robich, 9.11.98, 612.2-Randazzo, CLSM 04696 octo. 9) TRENTO, Cort Montagne, $1100 \mathrm{~m}$, on roe deer dung, M. Castoldi \& G. Robich, 3.5.99, 059.3-Tione di Trento, CLSM 04696 ena. 10) PISA, S. Rossore park, 0 m, on wild rabbit dung, F. Doveri, 5.3.00, 272.1-Migliarino, CLSM 04696 deca. 11) VERCELLI, Mount Rosa (Rio Endre peat bog), $2600 \mathrm{~m}$, on rock goat dung in d.c., G. Robich, 24.8.00, 071.2-Alagna Valsesia, CLSM 04696-XI. 12) AOSTA, Salati Pass, 3000 m, on rock goat dung in d.c., L. Levorato, 23.8.00, 071.3-Gressoney la Trinité, CLSM 04696-XII. 13) AOSTA, Bettaforca, $2800 \mathrm{~m}$, on rock goat dung in d.c., L. Levorato, 26.8.00, 071.3-Gressoney la Trinité, CLSM 04696-XIII. 14) RAVENNA, Lido di Savio, 0 m, on wild rabbit dung in d.c., Carchia, 6.4.01, 241.4-Lido di Savio, CLSM 04696-XIV. 15) COSENZA, Serra Pedace (loc. Silvana Manzio), $1450 \mathrm{~m}$, on cattle dung in d.c., C. Lavorato, 2.2.01, 560.1-Monte Volpintesta, CLSM 04696-XV. 16) COSENZA, Longobucco (loc. Pettinascura), $1580 \mathrm{~m}$, on sheep dung in d.c., C. Lavorato, 2.4.01, 560.1-Monte Volpintesta, CLSM 04696-XVI. 17) COSENZA, Acri (Manche di Greca), $1100 \mathrm{~m}$, on goat dung in d.c., C. Lavorato, 15.2.01, 552.3-Acri, CLSM 04696-XVII. 18) COSENZA, Mount Scuro, $1600 \mathrm{~m}$, on horse dung in d.c., C. Lavorato, 4.9.01, 560.3-Spezzano della Sila, CLSM 04696-XVIII. 19) MACERATA, Pintura di Bolognola, 1600 m, on cattle dung, F. Doveri, 3.5.02, 325.1-Bolognola, CLSM 04696XIX. 20) REGGIO CALABRIA, Gambarie, $1300 \mathrm{~m}$, on cattle dung in d.c., A. Bizzi, 17.10.03, 602.1-Gambarie, CLSM 04696-XX. 21) VERONA, Molina, 800 m, on horse dung in d.c., A. Bizzi, 18.9.03, 101.2-Dolcè, CLSM 04696-XXI. 22) VICENZA, Arsiero, Malga Melegnon, 1300 m, on cattle dung, A. Bizzi, 3.9.04, 081.1-Caldonazzo, CLSM 04696-XXII. 23) CUNEO, Viola, loc. il Colletto, $1100 \mathrm{~m}$, on cattle dung, A. Bizzi, 14.9.05, 227.2-Pamperato, CLSM 04696-XXIII. 24) BELLUNO, Canale d'Agordo, Geres, $1400 \mathrm{~m}$, on cattle dung in d.c., A. Bizzi, 26.6.05, 045.1Cencenighe Agordino, CLSM 04696-XXIV. 25) VENEZIA, Ca' Savio, 0 m, on hare dung in d.c., L. Levorato, 24.3.06, 128.3-Venezia, CLSM 04696-XXV. 26) TRENTO, Mount Baldo, malga Gambon, $1250 \mathrm{~m}$, on horse dung in d.c., A. Bizzi, 25.5.06, 080.2-Riva del Garda, CLSM 04696XXVI. 27) VERONA, Branchetto pass, $1600 \mathrm{~m}$, on cattle dung in d.c., A. Bizzi, 1.6.06, 102.3Bosco Chiesanova, CLSM 04696-XXVII. 28) TRENTO, Folgarida, Malga Folgarida, 1600 m, on cattle dung in d.c., F. Doveri, 6.9.07, 042.2-Lago di Tovel, CLSM 04696-XXVIII.

TOTAL 28: cattle 11; wild rabbit 5 ; horse 4 ; rock goat 2 ; donkey 1 ; goat 1 ; hare 1 ; roe deer 1 ; sheep 1 ; wolf 1 .

\section{Updated key to coprophilous Ascobolus from Italy}

(partly based on van Brummelen 1967 and Doveri 2004)

1) Ascomata superficial, small to very small, $<0.7 \mathrm{~mm}$ diam., discoidal or pulvinate from the beginning, or subglobose but only in an early phase, with the hymenial surface exposed from the first (gymnohymenial development according to van Brummelen 1967). Excipulum often reduced. Episporium reticulate, pitted, or echinulate

1*) Ascomata superficial or immersed, very small to large, up to $10 \mathrm{~mm}$ diam., always globose, subglobose or pyriform at first, so hymenial surface never exposed in an early phase (cleistohymenial development). Excipulum usually well developed. Episporium differently ornamented 
2) Ascomata white, pulvinate. Ectal excipulum scarcely developed, a textura globulosa-angularis. Asci amyloid, cylindric-claviform. Ascospores 13.5-15 × $8 \mu \mathrm{m}(14.5-17 \times 8-10$, van Brummelen 1967), ellipsoidal or narrowly ellipsoidal, warted-echinulate

sect. Heimerlia-A. aglaosporus (Figs 1-10)

2*) Ascomata usually dirty whitish, pulvinate or discoidal. Ectal excipulum a textura prismatica of cells perpendicular to the hymenial surface. Asci non-amyloid, broadly clavate to saccate. Ascospores usually broadly ellipsoidal (average $\mathrm{Q}=1.22$ ), sometimes subglobose or ellipsoidal, not echinulate (sect. Pseudascodesmis Brumm.)

3) Ascomata 400-700 $\mu \mathrm{m}$ diam. Asci 55-90 $\times 25-37 \mu \mathrm{m}$, usually 8-spored but only with two to six maturing ascospores. Ascospores pitted, 16-21 × 13-16 $\mu \mathrm{m}$................... A. perforatus (Figs 92-93) 3*) Ascomata 150-200 $\mu \mathrm{m}$ diam. Asci 130-160 × 30-35 $\mu \mathrm{m}, 8$-spored. Ascospores reticulate, 15$18 \times 13-14 \mu \mathrm{m}(13-19.5 \times 13-15.5$, van Brummelen 1967) .................. A. reticulatus (Figs 99-100) 4) Ascomata small, usually $<0.8 \mathrm{~mm}$ diam., remaining globose, subglobose, pyriform, turbinate also at maturity, sometimes immersed. Hymenial surface exposed during a late phase only ("telohymenial" according to van Brummelen, 1967) or rarely not exposed at all. Asci clavate, broadly clavate or saccate, rarely cylindric-clavate, usually with a dome-shaped apex. Ascospores ellipsoidal, exceptionally subglobose. Episporium smooth, dotted, or warted, sometimes with sparse, very thin crevices, never longitudinally striate ................ sect. Dasyobolus (Sacc.) Brumm. 5 4*) Ascomata larger, up to $10 \mathrm{~mm}$ diam., rarely less than $0.5 \mathrm{~mm}$ diam., often cup-shaped and flattening at maturity, superficial, with a hymenial surface exposed in an earlier phase ..................9

5) Ascomata brownish, yellow-brown or greenish yellow. Ascospores smooth or densely dotted,

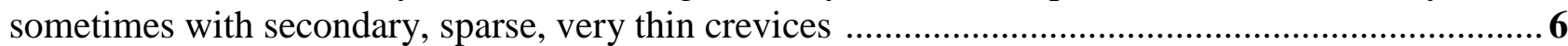

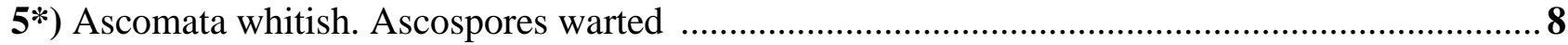
6) Ascomata immersed, erumpent at maturity. Asci broadly clavate to sacciform. Ascopores smooth, 56-63 × 31.5-38 $\mu \mathrm{m}(58-71 \times 28-36$, van Brummelen 1967) ......A. immersus (Figs 73-78) 6*) Ascomata superficial. Asci cylindric-clavate or clavate. Ascospores smaller, smooth or usually dotted

7) Ascospores 21-24 × 11-12 $\mu \mathrm{m}$ (19-23 × 10-12, van Brummelen 1967) ..A. mancus (Figs 82-87)

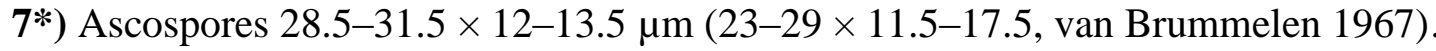

A. elegans (Figs 52-60)

8) Ascomata immersed with asci exposed. Asci clavate or clavate-saccate. Ascospores $25.5-28.5 \times$ 17-18 $\mu \mathrm{m}(26.5-30.5 \times 16-17.5$, van Brummelen 1967) ....................... stictoideus (Figs 109-112) 8*) Ascomata superficial. Asci clavate. Ascospores 14.5-20.5 × 8.5-10.5 $\mu \mathrm{m}$ ( 18.5-21 × 10-11.5, van Brummelen, 1967)

A. hawaiiensis (Figs 68-72)

9) Ascomata up to $0.5 \mu \mathrm{m}$ diam. (up to $1.5 \mu \mathrm{m}$, van Brummelen 1967), slowly expanding, sessile, lenticular, discoidal, pulvinate at maturity. Asci narrowly cylindric to cylindric-clavate. Ascospores spherical with rounded warts or truncate tubercles [sect. Sphaeridiobolus (Boud.) Brumm.], 11.5-13 $\mu \mathrm{m}$ diam. (10.5-13.5, van Brummelen 1967). Receptacles dirty whitish ..A. brassicae (Figs 32-38) 9*) Ascomata usually larger, soon expanding and becoming obconical or cup-shaped, sessile or pedunculate. Asci quite long, cylindric-clavate. Ascospores ellipsoidal or elipsoid-fusiform, exceptionally globose, often with longitudinal, sometimes anastomosing striae or crevices, rarely warted but never when globose, exceptionally dotted or smooth ........ sect. Ascobolus ss. Brumm. 10

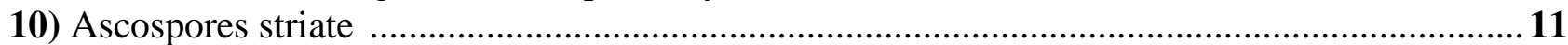

10*) Ascospores with a different ornamentation or smooth ...............................................18

11) Ascomata white or dirty whitish. Ascospores with longitudinal, sometimes anastomosing striae.

11*) Ascomata coloured, with a distinct margin. Ascospores longitudinally or transversely striate 13 12) Ascomata up to $0.4 \mathrm{~mm}$ diam., obconical to turbinate or subcylindric, smooth, lacking a differentiated margin. Ascospores 20.5-21.5 × 9.5-10.5 $\mu \mathrm{m}(20-36 \times 11-14$, van Brummelen, 1967) A. albidus (Figs 26-31) 12*) Ascomata up to $1.25 \mathrm{~mm}$ diam., obconical, cup- or saucer-shaped, finely granulose, with a 
distinct margin. Ascospores 18-19 × 8.5-9.5 $\mu \mathrm{m}(16-20 \times 9-10$, van Brummelen 1967)

A. sacchariferus (Figs 11-19)

13) Ascomata 0.4-0.7 mm diam., greenish yellow, finely granulose, with a crenulate margin. Ascospores densely and transversely striate, 11.5-12.5 × 6-7 $\mu \mathrm{m}(13-14.5 \times 7.5-8.5$, van Brummelen 1967) A. lineolatus (Figs 79-81)

13*) Ascospores larger, longitudinally striate.

14) Ascomata $0.7-1 \mathrm{~mm}$ diam., pinkish purple, smooth, usually with a regular margin. Ascospores 25-28.5 × 12-13.5 $\mu \mathrm{m}(18-28 \times 10-12$, van Brummelen 1967), with anastomosing striae

A. roseopurpurascens (Figs 101-108)

14*) Ascomata yellow, yellow-greenish or brown, furfuraceous or granulose, usually with a crenulate margin. Episporium with occasionally anastomosing striae

15) Ascomata up to $10 \mathrm{~mm}$ diam. Spores $24.5-29 \times 11-12.5 \mu \mathrm{m}(19-28 \times 10-14$, van Brummelen 1967) A. furfuraceus (Figs 61-67)

15*) Ascomata up to $3 \mathrm{~mm}$ diam. Ascospores smaller.

16) Ascomata sessile or substipitate, $0.7-1.3 \mathrm{~mm}$ diam. Ascospores $17-18 \times 9.5-10.5 \mu \mathrm{m}(17-22 \times$ 9.5-12, van Brummelen 1967), with 1-5 longitudinal or oblique striae on each side

A. michaudii (Figs 88-91)

16*) Ascomata 1-3 $\mathrm{mm}$ diam. Ascospores smaller, with 6-8 longitudinal, sometimes branched striae on each side

17) Ascomata yellow-greenish, sessile. Ascospores 12.5-16 × 7-9 $\mu \mathrm{m}(9.5-15 \times 6-8$, van Brummelen 1967)

A. crenulatus (Figs 48-51)

17*) Ascomata lemon yellow, stipitate. Ascospores 13-15.2 × 6.5-7.5 $\mu \mathrm{m}(13-15 \times 7.5-8.5$, van Brummelen 1967)

A. costantinii (Figs 43-47)

18) Ascomata $0.50-0.75 \mathrm{~mm}$ diam., violet, with a regular or slightly crenulate margin. Ascospores $13.5-15 \times 7-8 \mu \mathrm{m}$, dotted or with coarsely knobbed clusters of pigment. Gelatinous perisporium bilateral. Paraphyses with strongly inflated tips A. aff. pseudocainii (Figs 94-98) 18*) Ascomata up to $1.2 \mathrm{~mm}$ diam., whitish, with a scarcely differentiated margin. Ascospores 13.5-14 × 7-7.5 $\mu \mathrm{m}$, smooth, finally reticulate. Gelatinous perisporium unilateral. Paraphyses not or hardly inflated at the tips A. carletoni (Figs 39-42)

\section{Updated key to coprophilous Saccobolus from Italy}

(partly based on van Brummelen 1967 and Doveri 2004)

1) Ascomata pale to deep yellow or amber. Paraphyses containing yellow pigments. Ascospores paired in four rows parallel to the longitudinal axis of the ascus (van Brummelen's pattern I). Intercellular pigment absent in the excipulum and among the apices of paraphyses

sect. Saccobolus ss. Brumm. 2

1*) Ascomata whitish or coloured, never yellow. Paraphyses without yellow pigments. Ascospores with a different disposition. Intercellular pigment often present sect. Eriobolus Sacc. 7

2) Spore clusters rather loose, so ascospores becoming free at maturity. Ascospores 17-18.5 $\times 9.5-$ $10.5 \mu \mathrm{m}$ (16-19.5 × 7.5-9, van Brummelen 1967), ellipsoidal, with a dotted episporium, sometimes with few thin cracks S. saccoboloides (Figs 143-146)

2*) Spore clusters compact and ascospores remaining solidly cemented at maturity .......................3 3) Spore clusters contracted at maturity. Ascospores $15-15.5 \times 7-7.5 \mu \mathrm{m}(14-17.5 \times 7.5-8.5$, van Brummelen 1967), narrowly ellipsoidal with rounded ends, densely and finely dotted

S. truncatus (Figs 154-158)

$3^{*}$ ) Spore clusters not or hardly contracted at maturity. Ascospores with truncate ends

4) Ascomata citrine. Ascospores 20-22 × 9-9.5 $\mu \mathrm{m}$ (16-22 × 7.5-9, van Brummelen 1967), with strongly truncate ends, ornamented with fine isolated warts.

S. citrinus (Figs 121-124)

4*) Ascomata amber or sulphur yellow. Ascospores smooth to finely and densely dotted or verruculose, sometimes with irregular cracks 
5) Ascomata 0.15-0.35 mm diam. Ascospores dotted, 12-14 × 6-8 $\mu \mathrm{m}(11.5-13.5 \times 5.5-6.5$, van Brummelen 1967)

5*) Ascospores larger

6) Ascomata 0.4-1 mm diam. Ascospores 25.5-29 x 11.5-13 $\mu \mathrm{m}(22-29 \times 8.5-14.5$, van Brummelen 1967), smooth or dotted

S. glaber (Figs 132-138)

6*) Ascomata 0.1-0.2 mm diam. Ascospores 18-20 × 9.1-9.5 $\mu \mathrm{m}(18.5-20.5 \times 9-10$, van Brummelen 1969), densely dotted or very finely warted ........................ S. succineus (Figs 147-153) 7) Ascospores 12.5-13.5 × 6.5-7 $\mu \mathrm{m}$ (11.5-14.5 × 6.5-7.5, van Brummelen 1967), arranged in subglobose or ellipsoidal clusters (van Brummelen's pattern IV)

S. dilutellus (Figs 129-131)

7*) Ascospores disposed in three rows: one row of two ascospores and two rows of three (van Brummelen's pattern II)....

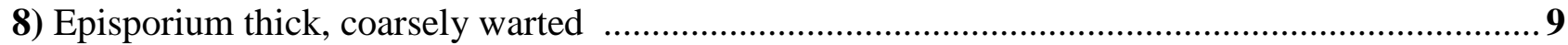

8*) Episporium thin, finely dotted or almost smooth ............................................................ 11

9) Episporium very thick, with warts or shapeless crusts of pigment up to $3 \mu \mathrm{m}$ high. Ascospores 20-21 ×9-10 $\mu \mathrm{m}(17.5-23 \times 8.5-10$, van Brummelen 1967), ellipsoid-fusiform, usually ventricose and inequilateral S. beckii (Figs 113-116)

9*) Episporium thinner, both with fine and coarse warts. Ascospores smaller 10

10) Ascomata pulvinate, pale brown, with a purple-brown to brown intercellular pigment in the excipulum. Spore clusters becoming longitudinally contracted at maturity. Ascospores $12.5-16 \times 7-$ $8 \mu \mathrm{m}(13.5-18 \times 7.5-9.5$, van Brummelen 1967), ellipsoidal or ovoidal to broadly fusiform (average $\mathrm{Q}=1.67$ ), sometimes slightly inequilateral S. obscurus (Figs 21-25) 10*) Ascomata subglobose, whitish, with a very pale violet intercellular pigment in the excipulum. Spore clusters not contracted at maturity. Ascospores 13-16 × 6-9 $\mu \mathrm{m}(14-16 \times 8-9$, van Brummelen 1967), ellipsoid-fusiform (average $Q=2.20$ ), often inequilateral

S. verrucisporus (Figs 159-162)

11) Ascomata tomentose, with isolated or fasciculate hairs, sometimes disposed in small triangular scales. Ascospores 18-19 × 7.5-8.5 $\mu \mathrm{m}$ (16-17.5 × 7-8.5, van Brummelen 1967)

S. caesariatus (Figs 117-120)

11*) Ascomata glabrous 12

12) Ascomata up to $1.5 \mathrm{~mm}$ diam. Excipulum usually with a violet intercellular pigment. Ascospores 18.5-19 × 7.5-8.5 um (13-21.5 × 6.5-9.5, van Brummelen 1967)

S. versicolor (Figs 163-165)

12*) Ascomata smaller, 0.15-0.27 mm diam. Excipulum without intercellular pigment. Ascospores 12.5-15 × 6.5-7 $\mu \mathrm{m}(10-14.5 \times 5-7.5$, van Brummelen 1967) S. depauperatus (Figs 125-128)

\section{Acknowledgements}

The author thanks L. Levorato and all friends who provided him with part of the material subject of this work.

\section{References}

Aas O. 1978a - Koprofile discomycetar (Ascomycetes: Discomycetes operculati = Pezizales) i Noreg. Universitetet i Bergen-Botanisk Institutt. Bergen.

Aas O. 1978b - Two new coprophilous species of Saccobolus. Norwegian Journal of Botany 25, 6568.

Barrasa Gonzales JM. 1985 - Contribución al estudio taxonómico, ecológico y corológico de los ascomycetes coprófilos en España. Universidad de Alcalá de Henares.

Bednarczyk MA. 1974 - Materials of the knowledge of the coprophilous fungi in the Lublin region. Acta Mycologica 10, 331-342.

Bell A. 2005 - An illustrated guide to the coprophilous ascomycetes of Australia. CBS Diversity Series (Utrecht) 3, 1-172. 
Bell A, Mahoney DP. 2007 - Ascobolus calesco sp. nov. Fungal Planet 21.

Brummelen J van. 1967 - A world-monograph of the genera Ascobolus and Saccobolus (Ascomycetes, Pezizales). Persoonia, supplement Vol. I. Rijksherbarium, Leiden.

Brummelen J van. 1969 - Studies on discomycetes-III. Persoonia 5, 225-231.

Caillet M, Moyne G. 1982 - Contribution à l'etude des Ascoboleae de Franche-Comté. Bulletin de la Société d'Histoire naturelle du Doubs 80, 41-61.

Cooke MC. 1876 - New British fungi. Grevillea 4, 109-114.

Crouan PL, Crouan HM. 1858 - Note sur neuf Ascobolus nouveaux. Annales des Sciences Naturelles, Botanique 4, 10, 193-199.

De Meulder H. 2000 - Onderzoek naar het voorkomen van ascomyceten op mest van Gallowayrunderen (deel 1). AMK Mededelingen 2, 32-37.

De Meulder H. 2007 - Onderzoek naar het voorkomen van paddestoelen op mest van geit en schaap (deel 1). AMK Mededelingen 1, 3-10.

Dennis RWG. 1981 - British Ascomycetes, 2 ed. J. Cramer, Vaduz.

Dissing H. 1989 - Four new coprophilous species of Ascobolus and Saccobolus from Greenland (Pezizales). Opera Botanica 100, 43-50.

Dissing H, Eckblad FE, Lange M. 2000 - Pezizales. In: Nordic Macromycetes 1 (Hansen L. \& Knudsen H. eds.). Copenhagen.

Dokmetzian DA, Forchiassin F, Ranalli ME. 1986 - Estudio sistemático y biológico de las ascoboláceas de Argentina. X. Saccobolus pampeanus sp. nov. Estudios de cultivo y citología. Physis C, 44 (106), 29-38.

Dokmetzian DA, Giménez MC, Cinto IE, Ranalli ME. 2004 - Systematic and biological study of Ascobolaceae of Argentina XIX. Two new species of Ascobolus (Ascomycota). Hickenia 3(49), 205-211.

Doveri F. 2004 - Fungi Fimicoli Italici. A.M.B., Vicenza.

Doveri F. 2007 - An updated key to coprophilous Pezizales and Thelebolales in Italy. Mycologia Montenegrina 10, 55-82.

Doveri F. 2011 - Addition to "Fungi Fimicoli Italici": An update on the occurrence of coprophilous Basidiomycetes and Ascomycetes in Italy with new records and descriptions. Mycosphere 2 (4), 331-427.

Doveri F. 2012 - An exceptional find on rabbit dung from Italy: third record worldwide of Ascobolus perforatus. Mycosphere 3 (1), 29-35. http://dx.doi.org/10.5943/mycosphere/3/1/3

Doveri F, Cacialli G, Caroti V. 2000 - Guide pour l'identification des Pezizales fimicoles d'Italie. Contribution à l'étude des champignons fimicoles - XXXII. Documents mycologiques 30 (117-118), 3-97.

Eckblad FE. 1968 - The genera of the operculate discomycetes. Nytt Magasin for Botanikk 15, 1192.

Ghadge DN, Patil MS. 1988 - Studies on Discomycetes IV-Genus Ascobolus. Indian Phytopathology $41,177-182$.

Häffner J. 1986 - Rezente Ascomycetenfunde III - Dungbewohner, Gärfutter- und Nadelstreubesiedler. Einführung in die Gattung Saccobolus. Mitteilungsblatt der Arbeitsgemeinschaft Pilzkunde Niederrhein 4, 106-129.

Heimerl A. 1889 - Die niederösterreichischen Ascoboleen. K. K. Ober-Realschule im Bezirke Sechshaus bei Wien 15, 1-32.

Jahn E. 1997 - Koprophile Pezizales von Dungkulturen aus dem norddeutschen Raum und aus verschiedenen Teilen der Erde. Zeitschrift für Mykologie 63, 133-148.

Jeng RS, Cain RF. 1976 - A new species of Ascodesmis from Venezuela. Mycotaxon 3, 391- 395.

Jülich W. 1968 - Über coprophile Pilze Mitteuropas I. Verhandlungen des Botanischen Vereins für die Provinz Brandenburg 105, 34-43.

Korf RP, Zhuang WY. 1991 - A preliminary discomycete flora of Macaronesia: part 12, Pyronematineae, and Pezizineae, Ascobolaceae. Mycotaxon 40, 307-318. 
Kutorga E. 2000 - Mycota Lithuaniae III, 5. Pezizales. Institutum Botanicae Lithuaniae Universitatis Vilnensis. Vilnius.

Larsen K. 1970 - The genus Saccobolus in Denmark. Botanisk Tidsskrift 65, 371-389.

Lohmeyer TR. 1995 - Pilze auf Helgoland. Zur Mykologie einer Ferieninsel in der Nordsee. Teil 1: Ascomyceten. Zeitschrift für Mykologie 61, 79-121.

Marchal E. 1895 - Champignons coprophiles de Belgique. VII. Bulletin de la Société Royale de Botanique de Belgique 34, 125-149.

Minoura K, Matsumura E, Morinaga T. 1978 - Notes on coprophilous Discomycetes in Japan (II). Transactions of the Mycological Society of Japan 19, 355-361.

Moravec J. 1970 - Operkulátní diskomycety celedi Ascobolaceae Sacc. z okresu Mladá Boleslav v Cechách. Česká Mykologie 24, 134-145.

Moyne G, Petit J. 2006-2007 - Quelques ascomycetes coprophiles recoltes en France et en particulier dans le departement du Boubs. Bulletin de la Société d'Histoire Naturelle du Doubs 91, 49-114.

Mungai PG, Njogu JG, Chukeatirote E, Hyde KD. 2012 - Studies of coprophilous ascomycetes in Kenya - Ascobolus species from wildlife dung. Current Research in Environmental \& Applied Mycology 2(1), 1-16.

Otani Y, Kanzawa S. 1970 - Notes on coprophilous discomycetes in Japan I. Transactions of the Mycological Society of Japan 10, 117-126.

Parker AD. 1979 - Associations between coprophilous Ascomycetes and fecal substrates in Illinois. Mycologia 71, 1206-1214. http://dx.doi.org/10.2307/3759107

Paulsen MD, Dissing H. 1979 - The genus Ascobolus in Denmark. Botanisk Tidsskrift 74, 67-78.

Piontelli EL, Cruz RC, Toro Santa Maria C, Toro Santa Maria MA. 2006 - Coprophilous fungal community of wild rabbit in a park of a hospital (Chile): a taxonomic approach. Boletín Micológico 21, 1-17.

Prokhorov VP. 1989 - The records of the discomycetes of the genera Ascobolus and Saccobolus from Estonia. Proceedings of the Academy of Sciences of the Estonian SSR. Biology 38, 24-32.

Prokhorov VP, Raitviir A. 1991 - New or interesting species of Ascobolus and Saccobolus in the U.S.S.R. Cryptogamic Botany 2-3, 205-213.

Raitviir A, Prokhorov V. 1988 - Discomycetes of Middle Asia. IV. New data on Ascobolaceae and Thelebolaceae. Proceedings of the Academy of Sciences of the Estonian SSR. Biology 37, 215-222.

Ranalli ME, Cinto RO. 1972 - Estudio sistemático y biológico de las Ascobolaceas de Argentina. IV. Boletín de la Sociedad Argentina de Botánica 14, 285-304.

Ranalli ME, Mercuri OA. 1995 - Taxonomy of some Argentine species of Saccobolus. Mycotaxon $54,315-327$.

Richardson MJ. 1998 - New and interesting records of coprophilous fungi. Botanical Journal of Scotland 50, 161-175. http://dx.doi.org/10.1080/03746609808684913

Richardson MJ. 2001a - Diversity and occurrence of coprophilous fungi. Mycological Research 105, 387-402. http://dx.doi.org/10.1017/S0953756201003884

Richardson MJ. 2001b - Coprophilous fungi from Brazil. Brazilian Archives of Biology and Technology 44, 283-289. http://dx.doi.org/10.1590/S1516-89132001000300010

Richardson MJ. 2004 - Coprophilus fungi from Iceland. Acta Botanica Islandica 14, 77-102.

Richardson MJ. 2005 - Coprophilous fungi from the Faroe Islands. Fróðskaparrit 53, 67-81.

Richardson MJ. 2007 - The distribution and occurrence of coprophilous Ascobolaceae. Mycologia Montenegrina 10, 211-227.

Richardson MJ. 2008 - Records of French coprophilous fungi. Cryptogamie, Mycologie 29, 157177.

Richardson MJ. 2011 - Additions to the coprophilous mycota of Iceland. Acta Botanica Islandica 15, 23-49.

Richardson MJ, Watling R. 1997 - Keys to fungi on dung. British Mycological Society. 
Schroeter J. 1893 - Die Pilze Schlesiens. In F Cohn (ed) - Kriptogamen-Flora von Schlesien 3 (2), 1256.

Seaver FJ. 1928 - The north-american cup-fungi (operculates). F.J. Seaver, New York.

Svrček M. 1957 - Príspevek k poznání ceskoslovenských diskomycetu z celedi Ascobolaceae. Česká Mykologie 11, 105-118.

Svrček M. 1959 - Über einige interessante Arten der im Jahre 1958 beobachteten koprophilen Pilzen. Česká Mykologie 13, 92-102.

Svrček M. 1962 - Diskomycety z Nízkých Tater, nalezené během posjezdové exkurze II. SEM. 1960. Česká Mykologie 16, 87-114.

Valldosera M, Guarro J. 1992 - Estudios sobre hongos coprófilos aislados en España. XVII. Ascomycotina. Boletín de la Sociedad Micológica de Madrid 17, 19-37.

Velenovský J. 1934 - Monographia Discomycetum Bohemiae. Pragae.

Vooren N van, Meyer M. 2010 - Saccobolus obscurus, une curiosité au sein du genre coprophile Saccobolus (Ascomycota, Pezizales). Cryptogamie, Mycologie 31, 155-159. 